\title{
Control Robusto de Reactores Químicos
}

\author{
presentada por \\ Alejandro Ricardo Femat Flores \\ Maestro en Ingeniería Química \\ Departamento de Ingeniería de Procesos e Hidráulica \\ División de Ciencias Básicas e Ingeniería \\ Universidad Autónoma Metropolitana - Iztapalapa \\ Av. Michoacana y Purísima s/n, col. Vicentina, cp 09340, \\ México D.F., MEXICO
}

1997

${ }^{\dagger}$ Asesor: Dr. José de Jesús Alvarez Ramírez 
eft todas las personas que

he quexido y estin muextas.

Poxque con las que auin viven

me seguire embriagando

a la luz de una vela.

OY acenca de las que vinixan,

sean bionvisidas,

con musica bailaxemos

en noctambulos desvelos.

Cl quienes vienen,

la bienvenida;

a quienes van,

la bienida.

(C) en todo casa,

al werues pexa con placex. 


\section{Epítome}

La presente tesis trata con el problema de control robusto para sistemas una entrada una salida UEUS (SISO, por sus siglas en inglés). En primer instancia se desarrolla un esquema de control basado en estimación discreta de incertidumbres en el cual todas las señales internas están acotadas. Esto permite garantizar estabilidad al menos semiglobalmente. El esquema robusto discreto presentado es capaz de estabilizar reactores químicos (o regular su salida) que puedan ser modelados por sistemas de primer orden y cuyo modelo sea incierto. A su vez, el esquema robusto discreto faculta para entender a las incertidumbres como una variable de estado y asi inducir un algoritmo continuo.

La versión en continuo del control asintótico robusto para sistemas de primer orden hace ver más claras las propiedades de robustez que presenta el control resultante del algoritmo de diseño. Más específicamente, con este algoritmo se pueden controlar procesos químicos a pesar de errores de modelado, variaciones paramétricas y perturbaciones en la planta mientras que el algoritmo discreto sólo es robusto ante errores en modelo. Bajo una interpretación clásica se entiende al control desarrollado como un controlador PI con capacidad de estimar y contrarrestar perturbaciones dinámicas en la entrada del proceso. Luego, se realiza una generalización del algoritmo, es decir, se presentan las modificaciones necesarias para estabilizar robusta y asintóticamente sistemas de orden superior.

El algoritmo de control está fundamentado en geometría diferencial y resultados recientes sobre linealización por retroalimentación de salida. De tal modo, se parte del procedimiento de linealización entrada-salida de sistemas no lineales para establecer una estrategia que resulte en un controlador rubusto. En concreto, la idea para manejar las incertidumbres estriba en aglutinarlas como una función no lineal. Dicha función no lineal es interpretada como una variable de estado en un sistema extendido, el cual es dinámicamente externamente equivalente al sistema no lineal incierto original. Una vez que las incertidumbres han sido interpretadas como una variable de, se diseña un observador de estados con el fin de reconstruir la dinámica del estado aumentado y consecuentemente de las incertidumbres. Entonces, el valor estimado del estado aumentado se utiliza en una ley de control de tipo linealizante para contrarrestar las acciones no lineales en la planta e inducirle a esta el comportamiento deseado mediante la imposición de polos. Así, el control resultante del algoritmo desarrollado comprende dos partes: (a) un compensador dinámico de incertidumbres dado por el estimador de estados para el sistema extendido y (b) una ley de control de tipo linealizante.

Tanto reactores continuos de tanque agitado (de polimerización, biológicos y catalíticos de lecho fluidizado) como intercambiadores de calor son usados para probar el desempeño del controlador resultante. La implementación fue llevada a cabo mediante simulaciones numéricas (a excepción de un caso en que se implementó a un biorreactor de escala piloto). El desempeño observado es muy aceptable. Aunque el algoritmo desarrollado fue elaborado para procesos de alimentación continua, éste puede ser usado para controlar procesos por lotes. Finalmente, algunas extensiones son tratadas para mostrar futuras lineas de trabajo. 


\section{Prefacio}

Este escrito ha sido organizado de tal modo que consta de seis partes (una introductoria, tres capítulos de resultados obtenidos, una de extensiones sobre los resultados donde se plantean las líneas de trabajos futuros y la última donde se discuten las observaciones concluyentes finales). En la introducción se presentan tanto las ideas básicas como las diferencias y similitudes de los resultados mostrados en cada una de las secciones subsecuentes. Entonces, la sección de introducción puede ser vista como una descripción complementaria puesto que, en cada sección, el resultado correspondiente es discutido bajo su propio contexto. Además, buscando claridad, en el primer capitulo se recuerdan brevemente las propiedades elementales de sistemas reactantes en procesos continuos. Se discuten las características principales en el control de procesos. Se repasan algunos conceptos sobre teoría de control necesarios para la estrategia propuesta en este proyecto de investigación. Empero, la mayoría del texto consiste en la presentación de los resultados obtenidos distribuidos en cuatro partes.

Cada una de las partes de resultados se basa en versiones modificadas de artículos publicados, aceptados o enviados para su revisión en revistas y congresos especializados (a excepción de la penúltima, el capítulo 5 , en cuyo caso los artículos correspondientes aún están en redacción). La modificación de los manuscritos originales únicamente estriba en la reestructuración de los resultados para el presente texto y algunas correcciones menores. La organización de los artículos es como sigue:

Capítulo 2: Control Robusto de Reactores Químicos Basado en Estimación Discreta de Tasa de Reacción.

- O. Monroy, J. Alvarez-Ramirez and R. Femat (1995), A mass Balance Strategy for the Robust Control of Anaerobic Digestion, $I^{\text {st }}$ Int. Workshop on Monitoring of A naerobic Digestion Proc., Lab. de Biotech. de L'enviorement, Francia.

- J. Alvarez-Ramirez, R. Suárez y R. Femat (1996), Control of Continuous-Stirred Tank Reactors: Stabilization with unknown reaction rates. Chem. Eng. Sci., 51, 4183.

- O. Monroy, J. Alvarez-Ramírez, F. Cuervo y R. Femat (1996), An Adaptive Strategy to Control Anaerobic Digester for Watewater Treatment, Ind. Eng. Chem. Res., 35, 3442.

Capítulo 3: Control Robusto de Reactores Químicos: Un esquema de control para plantas de primer orden'.

- R. Femat, J. Alvarez-Ramírez y M. Rosales-Torres (1996), Control Robusto de una Clase de Reactor Biológico, XVII Encuentro Nacional de la AMIDIQ, Puerto Vallarta Jal., México.

\footnotetext{
${ }^{1}$ Esta parte fue parcialmente finaciada por Industrias Negromex S.A. bajo el esquema de becas "Diego Bricio Hernández".
} 
- J. Alvarez-Ramirez, R. Femat y J. González-Trejo (1997), Robust Control of Uncertain FirstOrder Systems with Least Prior Knowledge, Chem. Eng. Sci, por aparecer.

- J. Alvarez-Ramirez, R. Femat y A. Barreiro (1997), A PI Controller with Disturbance Estimation, Ind. Eng. Chem. Res., por aparecer.

- J. Alvarez-Ramirez, R. Suárez y R. Femat (1997), Robust Stabilization of Temperature in Continuous-Stirred-Tank Reactors, Chem. Eng. Sci, 52, 2233.

Capítulo 4: Linealización Asintótica Robusta de Procesos Químicos ${ }^{2}$

- J. González-Trejo, J. Alvarez-Ramírez y R. Femat (1996), Diseño de un Esquema de Control en Dos Puntos para una Torre de Destilación de Alta Pureza. XVII Encuentro Nacional de la AMIDIQ, Puerto Vallarta Jal., México.

- J. Alvarez-Ramírez, R. Suárez y R. Femat (1996), Robust Control of a Class of Uncertain Nonlinear Systems in Strict-Feedback Form, European J. of Control. En Revisión.

- J. Alvarez-Ramirez, I. Cervantes y R. Femat (1997), Robust Controllers for a Heat Exchanger, Ind. Eng. Chem. Res., 36, 382.

- R. Femat, J. Alvarez-Ramírez y M. Rosales-Torres (1997), Robust Asymptotic Stabilization via Uncertainty Estimation: Regulation of Temperature in a Fluidized Bed Reactor. Comp. and Chem. Eng., En revisión.

- R. Femat, J. Alvarez-Ramírez, M. Rosales-Torres y R. Garrido (1997), Robust Asymptotic Regulation of Substrate in Biological Reactors: Design and Performance Comparison. Ind. Eng. Chem Res. En revisión.

Capítulo 5: Algunas Extensiones a los Resultados Obtenidos.

\section{Agradecimientos}

Deseo agradecer el financiamiento otorgado por CONACyT mediante el programa de becas-crédito. Además, quiero hacer un reconocimiento a Industrias Negromex S.A. por el esfuerzo realizado para apoyar estudiantes de posgrado en México y al Instituo Mexicano del Petróleo por su decisión de fortalecer la investigación nacional.

A. R. Femat Flores

Diciembre de 1997

México D.F., México

${ }^{2}$ Esta parte fue parcialmente finaciada por el Instituto Mexicano del Petróleo bajo el Proyecto FIES-95-93II. 


\section{Contenido}

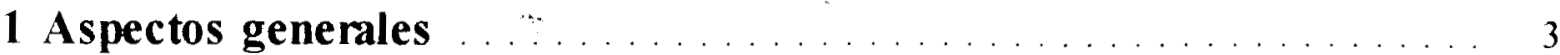

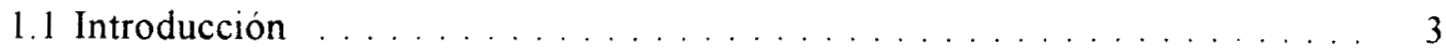

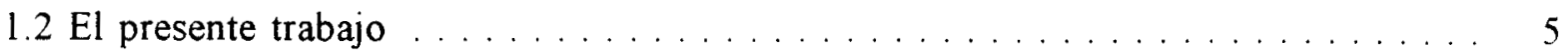

1.3 El compromiso de robustez-desempeño en control de procesos químicos . . 7

1.3.1 Sobre la formulación del problema de control . . . . . . . . . 8

1.4 Acerca de la dinámica de reactores de tanque agitado . . . . . . . . 10

1.5 Algunas definiciones elementales . . . . . . . . . . . . . . . . . 12

\section{Control Robusto de Procesos Químicos Basado en Estimación}

Discreta de Tasa de Reacción $\ldots \ldots \ldots \ldots \ldots \ldots \ldots \ldots \ldots$

2.1 Estabilización robusta de un reactor de alimentación continua $\ldots \ldots \ldots 15$

2.1.1 Un esquem a mbusto basado en diferencias finitas . . . . . . . . . . . 18

2.2 Reactor continuo de polimerización (un RCTA no isotérmico) . . . . . 20

2.3 Regulación robusta de sustrato en un digestor anaerobio . . . . . . . . . . 22

2.3.1 Una implementación experimental . . . . . . . . . . . . . . 24

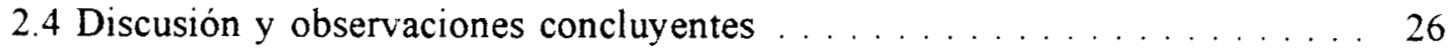

\section{Control Robusto de Reactores Químicos: Un esquema de control para}

plantas de primer orden . . . . . . . . . . . . . . . . . . . . 29

3.1 CAR de plantas de primer orden . . . . . . . . . . . . . . . . . . 29

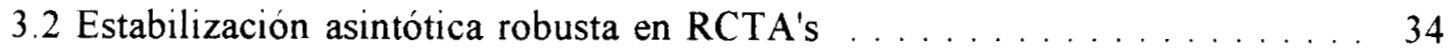

3.3 Una interpretación clásica del CAR . . . . . . . . . . . . . 39

3.4 Un ejemplo para comparar desempeño . . . . . . . . . . . . 43

3.4 .1 El controlador PI-IMC . . . . . . . . . . . . . . . . . . . 44

3.4 .2 Control adaptativo lineal . . . . . . . . . . . . . . . 45

3.4 .3 Control adaptativo no lineal . . . . . . . . . . . . 45

3.4 .4 El controlador asintótico robusto ............. 46

3.4 .5 Criterio de desempeño . . . . . . . . . . . . . . . . . . . 47

3.5 Observaciones y comentarios sobre el CAR para sistemas de primer

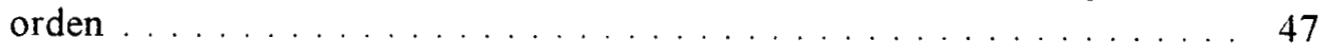

4 Linealización Asintótica Robusta de Procesos Químicos ........ . 51

4.1 Linealización de sistemas no lineales de orden superior . . . . . . . . 51

4.1.1 Linealización por retroalimentación de estados . . . . . . . . . . 51

4.1.2 Linealización por retroalimentación de mediciones . . . . . . . . . 53

4.2 Estabilización por retroalimentación de mediciones bajo campos vectoriales inciertos .......................... . . . . 54

4.2 .1 A nálisis de estabilidad . . . . . . . . . . . . . . . . . . 57

4.3 Linealización asintótica robusta de RCTA's exotérmicos .......... 59 
+3.1 Modelo dinámico del RLF ................... 60

4.3.2 La regulación robusta de temperatura del $R L F \ldots . . \ldots . . .61$

4.3.3 El desempeño del CAR en la regulación de temperatura . . . . . 64

4.4 Regulación de sustrato en una clase de fermentador ............ 66

4.4.1 El modelo dinámico del fermentador . . . . . . . . . . . . . . 67

+. +2 El diseño del controlador robusto para regulación de sustrato . . 68

4.4 La implementación numérica . . . . . . . . . . . . . . . . 70

4.5 Regulación robusta de temperatura en efluente de proceso . . . . . . . . 71

4.5.1 Modelo dinámico de un intercambiador de calor . . . . . . . . 72

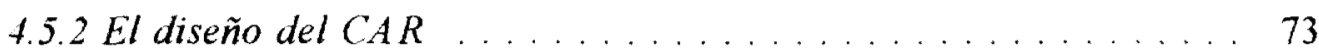

4.5 .3 Cálculo de los estimados requeridos para retroalimentación . . . . 75

4.5.4 Simulaciones numéricas . . . . . . . . . . . . . . . . . . . . 76

4.6 Comentarios sobre la linealización asintótica robusta $\ldots \ldots \ldots \ldots \ldots$

5. Algunas extensiones a los resultados obtenidos . . . . . . . . . . 82

5.1 Seguimiento robusto en una clase de sistema dinámico f........ 82

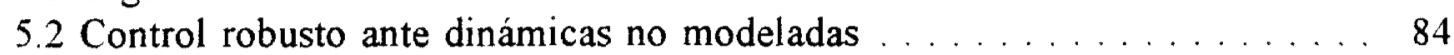

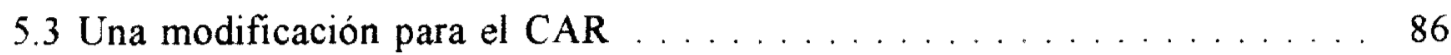

5.4 Observaciones sobre las extensiones discutidas $\ldots \ldots \ldots \ldots \ldots \ldots$

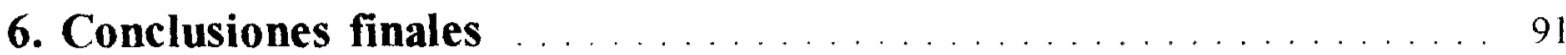

Apéndice $\mathbf{A}$ (Un resultado de estabilidad para una clase de sistemas variantes en tiempo)

Apéndice $\mathbf{B}$ (Un resultado de acerca de la observabilidad robusta) . . . . . . . . 95

Bibliografía $\ldots \ldots \ldots \ldots \ldots \ldots \ldots \ldots \ldots \ldots$

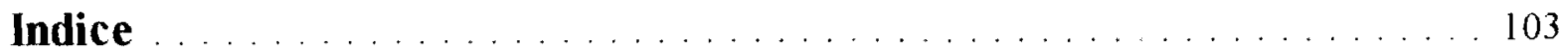

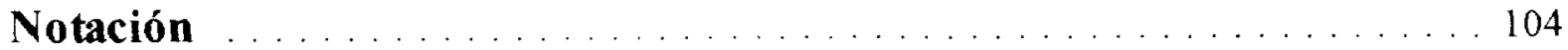


Aspectos generales

Capitulo Primero

\section{Aspectos Generales}


Capitulo Primero

La discusión presentada en este capitulo es abordada para establecer un puente en el lenguje utilizado en los campos de la teoría de control e ingenieria de reactores. Esto para introducir a algún lector en la problemática tratada en el proyecto de investigación desarrollado. 
Aspectos generales

\section{Aspectos generales}

\subsection{Introducción}

En las útimas dos décadas hemos sido testigos de un avance significativo en la teoría de control no lineal basada en geometría diferencial. En esencia, con los algoritmos propuestos para diseñar controladores, se pretende cancelar los términos no lineales del sistema e inducirle un comportamiento dinámico deseado. Una ley de control con tales características se conoce como linealizante.

Los procesos químicos son sistemas con características dinámicas que pueden ser explotadas mediante la teoría de control basada en geometría diferencial [1]. Académicamente, el problema de control en procesos químicos es por sí mismo de extremo interés, no sólo por las implicaciones tecnológicas, sino también, porque presenta retos interesantes desde el punto de vista de la teoría de control de sistemas no lineales. Por ejemplo, la estabilización de sistemas dinámicos en estados estacionarios inestables es un problema académico muy importante. Y qué decir de la regulación de comportamiento dinámico. Puesto que los sistemas reactantes pueden presentar tanto multiplicidad de estados estacionarios [2] como diversos comportamientos dinámicos (bifurcaciones, ciclos límite, caos, etc.) son propicios para estudiar su control. Por otra parte y desde un punto de vista práctico, en procesos continuos es necesaria la regulación de variables de salida (concentración, temperatura, etc.) en un estado estacionario deseado $\mathrm{y}$, en procesos por lotes, se requiere el seguimiento de trayectorias de alguna variable de interés (perfil de composición, peso molecular, viscosidad, etc.). Todo ello con fines económicos. La aplicación de teorías modernas de control a procesos químicos, ha beneficiado en una manera importante la calidad de productos de alto valor agregado. Y no sólo se requiere control de procesos para mejorar la calidad en producción, aparte de esto, por razones ambientales el uso de controladores es muy conveniente. En otras palabras, la teoría de control puede explotar el conocimiento desarrollado sobre procesos químicos y viceversa. La combinación de ambas áreas de estudio ofrece una excelente plataforma para establecer interesantes y completos proyectos de investigación científico-tecnológica.

Pues bien, como se dijo anteriormente, una linealización de sistemas por retroalimentación pretende cancelar las no linealidades del sistema a controlar con el fin de inducirle una dinámica deseada. Sin embargo, esto no es una tarea sencilla. Por un lado, es bien sabido que la obtención de modelos, que representen con exactitud el comportamiento dinámico de procesos químicos, es un trabajo arduo ( $\mathrm{y}$ a veces imposible). Es difícil entender y conocer con certeza, los fenómenos cinéticos y físicoquímicos (además de aquellos asociados con transporte de masa, energía y/o momento) involucrados en la transformación de materia. Adicionalmente, existen variaciones paramétricas en el tiempo (por ejemplo, los reactores no isotérmicos pueden tener cambios en los coeficientes de transferencia de calor debidos a incrustaciones en la pared de intercambio, o bien, en parámetros cinéticos que están asociados a la conversión o producción de alguna sustancia). Estas variaciones dificilmente pueden ser conocidas de una manera precisa. Por otra parte, la presencia de perturbaciones exógenas, tales como fluctuaciones en el caudal o composiciones de alimentación al proceso, 
son frecuentemente desconocidas y no medibles. Empero, a pesar de todas las incertidumbres mencionadas, se debe garantizar el control del proceso químico en cuestión (ya sea, estabilización o regulación para procesos de alimentación continua o seguimiento para procesos por lotes).

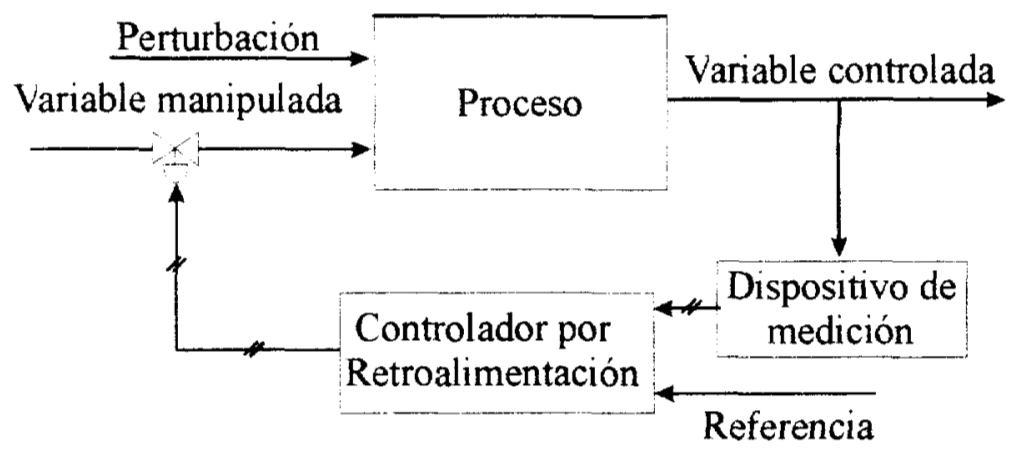

Figura 1. Diagrama de bloques para control retroalimentado en procesos químicos

Ahora, contemplemos desde la perspectiva de control a las incertidumbres antes mencionadas y que actúan sobre un sistema reactante. Considérese el diagrama de bloques de la Figura 1. Este incluye dos bloques llamados 'proceso' y 'controlador'. El controlador tiene como entradas la referencia y la salida del proceso (mediciones) y como su salida a la variable manipulada (la cual es una entrada al poceso, la otra es la señal perturbación). El influjo de la ruta paralela (i.e., bloque de retroalimentación) sobre el proceso es sustraer el efecto que tienen las entradas al proceso en su salida. Si el modelo reproduce exactamente el comportamiento dinámico del proceso químico, la perturbación podrá ser rechazada (o al menos se amortiguará su acción). Pero, si el modelo no reproduce fielmente el comportamiento dinámico del proceso, la señal de retroalimentación contendrá la influencia de la señal de perturbación (que no es medida) y el efecto del error en modelo (que es desconocido). Así que, el error en modelo puede generar problemas de inestabilidad. En otras palabras, esto obliga al diseñador a cambiar la idea de instrumentar acciones de control precalculadas por la idea de robustez. Además, marca una diferencia entre este concepto con el de desempeño.

Respecto a la técnica de diseño que ha de usarse, los controladores siempre son construidos con base en información (invariantemente incompleta) sobre el comportamiento dinámico del proceso. Esta información (es decir, el modelo) puede tener la forma de un sistema de ecuaciones diferenciales acopladas o simplemente puede estar representado por la transferencia de señal de la entrada a la salida del proceso (o quizá, sólo por la experiencia del operador). La exactitud de esta información varia, pero nunca es perfecta. Más aún, el comportamiento de la planta (léase proceso) cambia por sí mismo con el tiempo (por ejemplo, debido a cambios en actividad catalítica, condiciones hidrodinámicas, etc.). Por supuesto que estos cambios rara y dificilmente son capturados en el modelo. Asi que es deseable que el sistema a lazo cerrado sea insensible a estas incertidumbres. Al esquema que permite controlar un sistema a pesar de errores en modelo, variaciones paramétricas y/o perturbaciones en la 
planta se conoce como Control Robusto. Una solución para diseñar un control robusto consiste en desarrollar algoritmos que permitan una aproximación a las incertidumbres, y de este modo poder contrarrestar su efecto sobre el proceso. Por otra parte, la pretensión de robustecer un controlador puede acarrear problemas con efectividad y esfuerzo de las acciones de control. Así pues, se debe reducir el margen de robustez para lograr acciones de control suaves y del menor esfuerzo posible. A la combinación de estos dos requerimientos, suavidad y bajo esfuerzo, se le conoce como Desempeño [23].

\subsection{El presente trabajo}

En el presente trabajo se desarrollan algoritmos de diseño que resulten en controladores robustos (ante errores en modelo, variaciones paramétricas y perturbaciones en la planta) para sistemas una entrada una salida, (SISO por sus siglas en inglés). El controlador es diseñado a partir de un modelo del proceso obtenido mediante balances de masa, calor y movimiento (principios fundamentales). Así que el modelo está representado por un sistema de ecuaciones diferenciales acopladas. Los distintos algoritmos de diseño presentan diferencias en el mecanismo de estimación de incertidumbres y las herramientas matemáticas utilizadas, tanto para el diseño mismo como para probar la estabilidad del sistema a lazo cerrado. Lo anterior es debido a las características de las clases de sistemas que modelan cada proceso químico en particular. Sin embargo, en esencia todos los esquemas de control presentados son iguales. Es decir, tienen peculiaridades similares y parten de la misma idea.

La idea fundamental es aglutinar a las incertidumbres en una función no lineal, la cual puede ser interpretada como una variable de estado. Luego, se utiliza un esquema de estimación/observación para obtener un valor estimado de éste $\mathrm{y}$, consecuentemente, de las incertidumbres. De este modo, una ley de control de tipo linealizante es provista del valor estimado de las incertidumbres, por medio de la nueva variable de estado. Así pues, la aproximación propuesta consta de dos partes: (a) un estimador de incertidumbres (compensador dinámico) y (b) una ley de control de tipo linealizante. El texto ha sido organizado de forma tal que, para cada una de las diferentes clases de sistemas químicos tratados en su sección correspondiente, queden claras las similitudes entre el respectivo controlador y aquellos presentados en otras secciones. Otra característica del texto es que se redactó en forma inductiva, es decir, que conforme el lector avance en el texto le resulte natural el paso presentado en el siguiente esquema de control. Aunque la tesis está enfocada a reactores químicos, se rucurrirá a otro tipo de procesos (como intercambiadores de calor) con el fin de mostrar algunas características del conrolador asintótico robusto propuesto. Para ello, los capítulos han sido organizados como sigue.

En el segundo capítulo, se trata con sistemas de primer orden para los cuales es posible establecer un estimador de incertidumbres aglutinadas mediante un esquema de aproximación discreta. Este esquema presenta excelentes propiedades en la estabilización de estados estacionarios inestables, y además, en la regulación de una variable de salida para reactores químicos donde la tasa de reacción no está bien establecida (por ejemplo, reactores de polimerización, reactores de digestión anaerobia o reactores catalíticos). La estimación se realiza utilizando mediciones de la varible de salida y conociendo la acción de control 
inmediata anterior. El esquema de aproximación discreta se basa en diferencias finitas centrales. Es posible probar que el control resultante es un estabilizador práctico [3]. Más aún, se ha mostrado experimentalmente que la implementación de la estrategia es viable [4]. Dado que: (i) la estabilización (o regulación) se lleva a cabo usando únicamente la minima información disponible y, (ii) el sistema a lazo-cerrado es estable, entonces, se puede asegurar que el controlador resultante con estimación discreta de incertidumbres es robusto ante errores en modelo. En cuanto a desempeño, un único parametro permite ajustar al estimador para obtener acciones de control aceptables.

El tercer capítulo también versa sobre el control de sistemas de primer orden. Pero ahora, el esquema de estimación es continuo. Otra diferencia entre el algoritmo presentado en el segundo y tercer capitulo, consiste en que para el último: (i) es mucho más evidente el aglutinamiento de incertidumbres y, (ii) la interpretación de la función no lineal aglutinante, como una variable de estado en un sistema 2-dimensional (sistema extendido), resulta obvia. Es sobre el sistema extendido que se realiza la estimación de estados. Así, se pueden aproximar incertidumbres que incluyan variaciones paramétricas, errores en modelo y perturbaciones (tanto exógenas como endógenas). El estimador de estados que se propone es un observador de tipo Luenberger. Una de las ventajas que presenta el uso de esta clase de observador es el hecho que el controlador resultante es lineal. Dado que el controlador es lineal, se puede transformar mediante el operador de Laplace. De esta manera, es posible obtener la función de transferencia del control. Es ahi, en dominio de frecuencia, que se puede entender al control asintótico robusto (CAR) propuesto como un control proporcional-integral, PI, con estimacion dinámica de perturbaciones [5]. Se demuestra que bajo el CAR, el sistema a lazo cerrado es estable [6],[7]. Ya ha sido ilustrado el diseño de este estabilizador práctico robusto en su aplicación a reactores químicos con tasa de reacción desconocida [6] y en el control de composición en un punto de una columna de destilación [7].

En el cuarto capítulo se hace la descripción del algoritmo para el diseño de un CAR en sistemas de $n$-ésimo orden. Se parte de suposiciones prácticas asumiendo lo siguiente: (i) el grado relativo del sistema está bien definido para todo tiempo mayor que cero, (ii) se dispone tanto de información de diseño del equipo (valores nominales) como de mediciones de la variable de salida y, (iii) el sistema es de fase mínima. Desde el punto de vista de teoría de control, la primera es una suposición fuerte. Empero, en base al conocimiento fenomenológico de sistemas reactantes se puede justificar tal supuesto, más aún, en el último capitulo esta hipótesis será relajada. Respecto a la segunda, se puede asegurar que es práctica. Actualmente se cuenta con dispositivos de medición eficientes. Cabe recordar que se trata con sistemas SISO, por ello sólo se usará una medición (la salida). En lo referente a la información de diseño de equipo, es evidente que se dispone de valores nominales de parámetros (y puede ser que a partir de la experiencia de la operación del proceso se cuente con expresiones algebraicas heurísticas de variaciones de estos, las cuales no serán usadas aquí). Por ejemplo, el máximo coeficiente de transferencia de calor es aquel que se obtiene cuando el equipo está nuevo, lo cual significa que se conocen el valor máximo y mínimo (que es cero) de ese parámetro, no así su variación en tiempo debida a incrustaciones o ensuciamiento. $\mathrm{O}$ bien, el flujo nominal de alimentación a un reactor es conocido, sus fluctuaciones no. La suposición que asume fase mínima, significa que la dinámica de los 
estados no observables es estable. Esta hipótesis es la más fuerte, sin embargo será probada a lo largo del texto y cuando sea necesario. En realidad sólo se requiere que todas las señales internas estén acotadas.

Aunque en el cuarto capítulo es donde se realiza una generalización del algoritmo para diseño del CAR basado en estimación de incertidumbres, en el penúltimo capítulo se presentan algunas extensiones importantes. Las extensiones realizadas son: (i) el problema de control en sistemas no lineales inciertos con grado relativo no bien definido, (ii) el control robusto de sistemas no lineales en presencia de dinámicas no modeladas y, (iii) el CAR a pesar de mediciones ruidosas.

Es bien sabido que si el grado relativo no está bien definido, se deben estimar las derivadas de la entrada de control con el fin de linealizar la planta, es decir, el sistema es no causal. Dicho de otra forma, es necesario conocer el valor de la entrada de control para calcular esta misma, lo cual es un gran inconveniente. Con la aproximación propuesta (estimación de incertidumbres aglutinadas), no se requiere información alguna sobre dichas derivadas para calcular la entrada de control, pues resulta que estas derivadas son aglutinadas dentro de la función no lineal. Por consiguiente, en la estimación del estado aumentado se incluye implícitamente un estimado de ellas. Para esta extensión en particular, se usa un ejemplo sencillo, sin significado en procesos químicos, pero muy ilustrativo. En cuanto a la extensión asociada con dinámicas no modeladas, se presenta un caso de estudio (un intercambiador de calor para regular temperatura efluente de un proceso). Luego de eso, se muestra el efecto de mediciones ruidosas en la degradación del desempeño del CAR y se propone una modificación para mejorar el desempeño de las acciones de control.

El último capitulo presenta las conclusiones generales y la discusión de las líneas a seguir en trabajos futuros. Unos cuantos apendices sirven para esclarecer conceptos y resultados interesantes relacionados con el trabajo realizado.

\subsection{El compromiso de robustez-desempeño en control de procesos químicos}

Dada la enorme diversidad y complejidad de procesos químicos, resulta físicamente imposible desarrollar un controlador que trabaje con desempeño aceptable para todos. Por ejemplo, las características de comportamiento dinámico de una columna de destilación binaria son extremadamente diferentes a una fraccionadora de petróleo (como en perturbaciones en flujo, composición, cambios de referencia, equilibrio entre fases, etc.) y ambas son muy distintas a los reactores químicos, pues en estos últimos existen fenómenos que no necesariamente se presentan en destilación y viceversa (como difusión, desorción, cinética química, o bien, exotermicidad).

Aún dentro de un mismo tipo de proceso químico, diferentes peculiaridades hacen probable que la implementación de un controlador, diseñado especificamente para una clase de proceso químico, no presente un desempeño aceptable cuando se implementa en cualquier

otra. Pensemos en reactores químicos por ser el tipo de proceso que interesa al presente trabajo de investigación. Las características de un reactor tubular son muy diferentes a las de 
uno de tanque agitado [2]. El comportamiento dinámico del primero está gobernado por ecuaciones diferenciales parciales (o funciones de transferencia con tiempo de retardo, es decir, sistemas de dimensión infinita) mientras que el segundo puede ser modelado por ecuaciones diferenciales ordinarias (o bien operadores de transferencia entrada-salida con tiempo de retardo cero, i.e, sistemas de dimensión finita). Especificamente, en este trabajo de investigación se trata a una clase de reactor químico, el de tanque agitado. Aún el control en reactores de tanque agitado toma sentido de acuerdo con la clase de alimentación del proceso (continua o por lote).

En procesos de alimentación continua, las acciones de control están encaminadas a lograr la estabilización del proceso en un estado estacionario deseado, o bien, regular su salida con el fin que el efluente quede dentro de especificaciones. Para el caso de alimentación por lote, el control cobra sentido en el seguimiento de la trayectoria de algún estado en particular (por ejemplo, la concentración de producto, el peso molecular de polímero, viscosidad, etc.). Así que, desde el punto de vista de control, el problema debe ser abordado en forma distinta.

No obstante, con una misma base en los fundamentos teóricos apropiados, se puede llegar a establecer un algoritmo de diseño cuyos controladores resultantes posean propiedades semejantes, una vez que se haya definido correctamente el problema de control. Por ejemplo, la estabilización en un estado estacionario por medio de retroalimentación de mediciones.

\subsubsection{Sobre la formulación del problema de control}

De acuerdo con todo lo anterior, para algún procedimiento de diseño que genere un algoritmo de control que trabaje satisfactoriamenete en un 'sistema real', debe considerarse lo siguiente:

- El modelo del proceso: El modelo del proceso debe describir las principales característica dinámicas del sistema químico en cuestión. $Y$ deben estar claras estas propiedades dinámicas. Como, por ejemplo, el tipo de expresión que modela la tasa de conversión y las propiedades que posee.

- La descripción de las incertidumbres: Información acerca de la fuente que da origen a la incertidumbre del proceso. Sus propiedades y efectos.

- Tipos de entrada al proceso: Las caracteristicas de referencias (dinámicas o estáticas) y perturbaciones.

- Objetivos de desempeño: Pese que el criterio de desempeño es subjetivo, se han de establecer márgenes de tolerancia o desempeño nominal (especificaciones de efluente). Para que sin perder estabilidad y garantizando robustez se obtengan acciones de control suaves y de bajo esfuerzo.

La omisión de cualquiera de los cuatro aspectos anteriores pueden llevar a fallas del controlador cuando sea aplicado prácticamente. Tal vez el mejor modelo sea una combinación 
entre la experiencia de quien conoce el proceso y la representación matemática de la dinámica del sistema químico, empero la primera parte de esta combinación es en extremo subjetiva. Así que únicamente se puede tratrar de una forma sistemática con la segunda. Ignorar la incertidumbre en modelo puede producir controladores muy justos, cuyas acciones de control se muestren castañeantes (con un comportamiento "nervioso"), y peor aún, con fuertes "bandazos". Esto puede degenerar en inestabilidades.

Por otra parte, es fisicamente imposible diseñar controladores que trabajen "bien" para todas las entradas de control y perturbaciones en proceso. El diseñador debe decidir qué tipo de entradas (tanto al control como al proceso) son las más frecuentes e importantes y aceptar un desempeño inferior si las entradas en la operación real no son iguales a aquellas supuestas en el diseño. Finalmente, el diseñador debe especificar el significado de "buen desempeño" para un problema de control en particular. Lo que es bueno para algún caso puede ser inaceptable para otro.

Así pues genéricamente se puede formular lo siguente: El problema de control tratado aqui consiste en diseñar una ley de control por retroalimentación $u=u\left(y ; \pi_{\mathrm{c}}\right)$, donde y es la salida (medición) del proceso y $\pi_{\mathrm{c}}$ es un conjunto de parámetros de control. que estabilice al modelo del proceso cerca de una referencia (estabilidad práctica) con todas las señales intemas acotadas. A pesar de errores en modelo, variaciones paramétricas y perturbaciones actuantes sobre el sistema y además con acciones de control suaves y de bajo esfuerzo.

Con respecto a la representación del proceso, el diseño del controlador se llevará a cabo a partir de un modelo en dominio de tiempo. Un sistema en espacio de estados de la planta cuyas características y comportamiento dinámico se ilustran y discuten en la siguiente sección. En cuanto a las incertidumbres, se enfocará la atención a: (i) Errores en modelo. Se asumirá que las propiedades del modelo cinético de reacción se conocen. Sin embargo se supondrá que se ignoran las hipótesis hechas en la obtención de las expresiones cinéticas (por ejemplo, agrupación de especies químicas en pseudo-especies, isotermicidad cuando en realidad hubo fluctuaciones de temperatura en el experimento, mecanismo controlante en el proceso, etc.) (ii) Variaciones paramétricas de la planta: Se pondrá particular interés en variaciones asociadas con los paramétros cinéticos y de transferencia de calor. Se asumirá que se dispone de valores nominales (a partir del diseño del equipo) y de alguna información mínima sobre las cotas. (iii) En lo que concierne a las perturbaciones exógenas, se asumirá que estas son suaves y acotadas, lo cual se fundamenta en el siguiente argumento. Las perturbaciones más frecuentes e importantes a la entrada del procesos químicos son las fluctuaciones de caudal de alimentación, variaciones de composición alimentada, modifícación de las propiedades del medio enfriante/calentante, fluctuaciones en el caudal del medio enfriante/calentante, etc. Todas ellas son variaciones suaves en el tiempo y están acotadas. (iv) El objetivo de desempeño consiste en lograr mantener dentro de especificaciones (es decir que el sistema sea asintóticamente prácticamente estable) con acciones suaves y el menor esfuerzo de control posible. 
Capitulo Primero

\subsection{Acerca de la dinámica de reactores de tanque agitado}

En esta sección se recordarán brevemente los fundamentos de un sistema reactante representado por un reactor continuo de tanque agitado (RCTA). En la Figura 2 se muestra el conocido esquema de este proceso. Con base en principios fundamentales (es decir, balance de masa, momento y/o energia) y bajo condiciones de mezclado perfecto se obtiene el sistema de ecuaciones diferenciales ordinarias que modela al RCTA [2]. Así pues, el modelo puede escribirse en la siguiente forma:

$$
\begin{gathered}
\dot{x}=f(x, t)+g(x, t) u \\
y=h(x, t)
\end{gathered}
$$

donde $x=\left(x_{1}, x_{2}\right) \in \mathbf{R}^{\mathrm{n}}$ es un vector de estados, $x_{1} \in \mathbf{R}_{+}^{\mathrm{m}}$ es el vector de concentraciones y $x_{2}$ $\in \mathbb{R}^{\mathrm{n}-\mathrm{m}}$ el vector de estados medibles (por ejempo la temperaturas en el interior del reactor y/o chaqueta). $f(x, \mathrm{t})$ y $\mathrm{g}(x, \mathrm{t})$ son campos vectoriales suaves, $u \in \mathbf{R}$ es la entrada de control, $y \in$ R es la salida del sistema y $h(x, t)$ es una función suave. Cabe mencionar que existe un subconjunto $\mathbf{M} \subset \mathbf{R}_{+}^{\mathrm{m}+1}$ donde la dinámica de los estados $x_{1}$ está definida. De hecho, $\mathrm{M}=$ $\left[0, x_{1}^{\text {max }}\right] \times \mathbb{R}_{\text {, donde }} x_{1}^{\max } \in \mathbb{R}_{+}^{\mathrm{m}}$ son las máximas concentraciones posibles bajo los flujos de alimentación y condiciones de reacción dadas $\left(\left\|x_{1}^{\max }<x\right\|\right)[2]$.

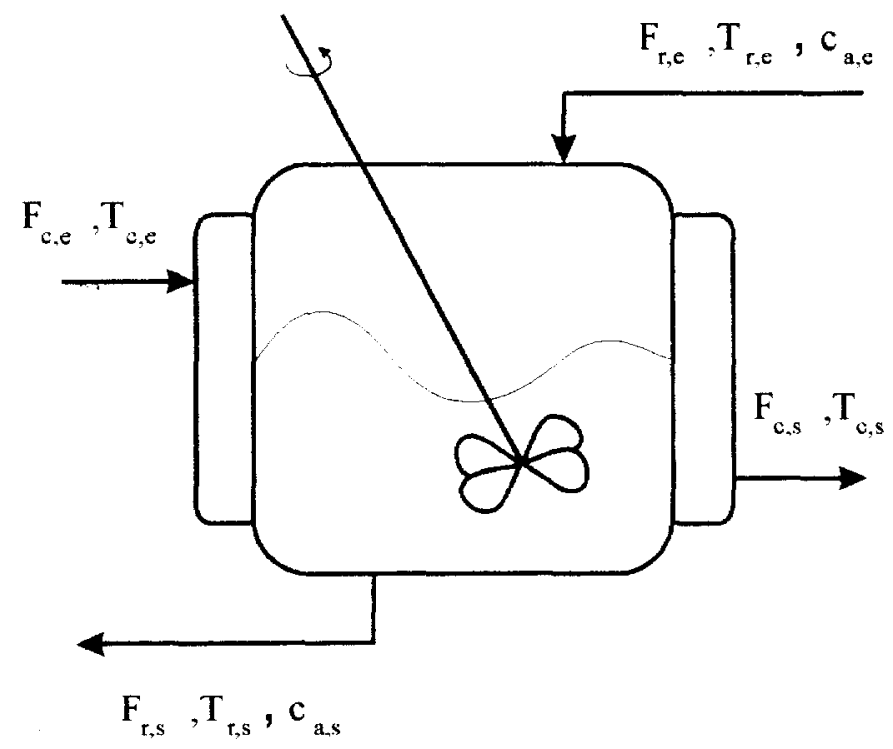

Figura 2. El Reactor Continuo de Tanque Agitado

Además del modelo de RCTA, el de otros procesos puede ser escrito como el sistema (1). Por ejemplo, un intercambiador de calor [8]. En muchos otros, un modelo de referencia obtenido mediante la identificación del operador de transferencia entrada-salida, seguida de una realización de estados, puede ser llevado a la forma de (1). Tal es el caso de una columna rectificadora [5]. Por supuesto que en los procesos donde no se lleva a cabo una reacción química, las no linealidades estarán asociadas a otra clase de fenómenos (por ejemplo, el 
Aspectos generales

equilibrio vapor-liquido en una columna de destilación). En este texto algunos procesos diferentes al RCTA serán usados para mostrar algunas propiedades del controlador propuesto. Las aclaraciones y definiciones pertinentes se realizarán cuando sea necesario.

Dado que diferentes sistemas químicos tienen modelos dinámicos que pueden ser escritos en la forma de (1). Entonces, si uno es capaz de establecer un algoritmo para diseñar un control robusto para el sistema (1), dicho esquema podrá ser utilizado en el control de procesos. Por último, una aclaración. Por simplicidad y sin pérdida de generalidad, en las siguientes secciones la dependencia del tiempo será omitida. En su caso, se hará la aclaración pertinente.

Ejemplo 1.1. (Un caso de RCTA, su dinámica y multiplicidad de estados estacionarios [9]). Consideremos que en el RCTA se lleva a cabo una reacción exotérmica $\mathrm{A} \rightarrow \mathrm{B}$ con una tasa de conversión tipo Arrhenius de primer orden $R(x)=\xi_{1} x_{1} \exp \left(-\mathrm{Ea} / \mathrm{R} x_{2}\right)$, donde Ea es la energía de activación, $R$ es la constante de los gases y $\xi_{1}$ es conocida como la constante de reacción (preexponencial cinética). Luego de hacer el balance de masa y calor (sin considerar la dinámica de la chaqueta de enfriamiento), se tiene el sistema dado por

$$
\begin{gathered}
\dot{x}_{1}=\theta\left(x_{1, \mathrm{e}}-x_{1}\right)+R(x) \\
\dot{x}_{2}=\theta\left(x_{2, \mathrm{e}}-x_{2}\right)-\beta(\mathrm{x}) R(x)+\gamma\left(x_{2}-u\right)
\end{gathered}
$$

Tomando los siguientes valores de los parámetros involucrados: (tiempo de residencia) $\theta=1.0$, (coeficiente de transferencia de calor) $\gamma=1.0$, (concentración de alimentación al reactor) $x_{1, \mathrm{e}}=1.0$, (temperatura de alimentación al reactor) $x_{2, \mathrm{e}}=350$, (término preexponencial) $\xi_{1}=\exp (25)$, (activación de conversión) $\mathrm{Ea} / \mathrm{R}=1 \times 10^{4}$, (entalpía de reacción) $\beta(x)=200$ y (temperatura de enfriamiento) $u=350$. El sistema anterior tiene tres puntos de equilibrio: uno inestable (un punto silla) con coordenadas en el espacio fase $\left(x_{1}^{*}, x_{2}^{*}\right)=(0.5,400)$ y dos estables (ignición y extinción) [2].

Por otro lado, para el caso isotérmico (i.e., $\dot{x}_{2}=0$ ) la dinámica del RCTA del ejemplo 1.1 estará representada por $\dot{x}_{1}=\mathrm{L}\left(x_{1}\right)+R\left(x_{1} ; x_{2}^{*}\right)$ la cual sólo tiene un punto de equilibrio, donde $\mathrm{L}\left(x_{1}\right)$ es una función lineal que esencialmente representa la residencia de $x_{1}$ en el reactor, y $\mathrm{R}\left(x_{1} ; x_{2}^{*}\right)$ representa la tasa de reacción a la temperatura constante, $x_{2}^{*}$. Más aún, la presencia de la dinámica de la temperatura de chaqueta puede inducir bifurcaciones $\mathrm{y}$ comportamiento anómalo en algún RCTA [10], [11], [12].

Además del caso de multiplicidad de estados estacionarios mostrado en el ejemplo anterior, por un lado, se sabe que en presencia de reacciones consecutivas la cantidad de estados estacionarios puede aumentar [2] y hasta se puede desplegar comportamiento caótico [11]. Por otro lado, nótese que para el caso del RCTA modelado por un sistema bidimensional (es decir bajo la suposición que la dinámica de la chaqueta de enfriamiento es despreciable), la variable manipulada es la temperatura de la chaqueta. Pero considerando la dinámica de la chaqueta de enfriamiento el modelo del RCTA es un sistema tridimensional y la variable manipulada es el caudal de enfriante que entra a la chaqueta. En este caso, el comportamiento del RCTA es, por mucho, más complejo (ver [9] y [10]). 
Capitulo Primero

\subsection{Algunas definiciones elementales}

Antes de abordar el problema planteado en la sección anterior, retomemos algunas definiciones elementales.

Definición 1.- Considere la siguiente función $f(z ; \pi)$ donde $z \in \mathbb{R}^{p}$ y $\pi$ es un conjunto de parámetros. Se dice que $f(z ; \pi)$ es localmente Lipschitz si existen unas constantes $\beta_{1}$ y $\beta_{2}$ (independientes de $t$ ) tales que $\|f(z ; \pi)-f(0 ; \pi)\| \leq \beta_{1}\|z\|+\beta_{2}$ para una vecindad de toda $z$ en una vecindad de $z=z^{*}$ y todo $t \geq 0$, donde $z^{*}=0$ es el equilibrio de $\dot{z}=f(z, v)$. Se dice que $f(z, v)$ es globalmente Lipschitz, si la propiedad anterior se mantiene para todo $z \in \mathbb{R}^{p}$ y todo $\mathrm{t} \geq 0$.

Definición 2.- Considérese un sistema $\dot{z}=f(\mathrm{z})+\mathrm{g}(\mathrm{z}) \boldsymbol{u}$ donde $u=u(\mathrm{z}, \mathrm{t})$ es una entrada de control. Se dice que el anterior sistema es intemamente estable si ante señales acotadas de $u$, el sistema a lazo cerrado genera señales internas acotadas.

Definición 3.- Considérese el sistema dinámico dado por

$$
\begin{gathered}
\dot{z}=f(z, v)+g(z, v) u \\
\dot{v}=\zeta(z, v)
\end{gathered}
$$

donde $z \in \mathbb{R}^{\rho}$ y $v \in \mathbb{R}^{n-p}$. Asúmase que $z^{*}=0$ es un punto de equilibrio. Se dice que el sistema anterior es de fase mínima si, en $z=z^{*}$, el subsistema dinámico $\dot{v}=\zeta(z, v)$ es global y asintóticamente estable. $Y$ es débilmente fase minima si es estable en el sentido de Lyapunov, i.e., existe una función positiva definida, $\mathrm{C}^{2}, \mathrm{~W}(\mathrm{z})$ tal que $\mathscr{L}_{\mathrm{f}} \mathrm{W} \leq 0$ en una vecindad de $\mathrm{z}=$ $z^{*}$, donde $\mathscr{L}_{f} \mathrm{~W}$ es la derivada de Lie de $\mathrm{W}(\mathrm{z})$ respecto a $f(z, v)$.

Definición 4.- Se dice que un sistema a lazo cerrado es prácticamente estable si para todo conjunto $\Sigma \subset M$ que contenga a la referencia $x^{*}$, si existe una ley de control (estabilizador práctico) la cual hace de este conjunto el atractor estable del sistema en una vecindad $\delta(\Sigma) \subset M$ de $\Sigma$, i.e., la trayectoria del sistema de control que inicie dentro de $\delta(\Sigma)$ converge a $\Sigma$.

Definición 5. - Una función $f: \mathbb{R}_{+} \rightarrow \mathbb{R}_{\text {}}$ se dice que es de clase $\mathrm{K}$ si es continua, estrictamente creciente y satisface $f(0)=0$. Y es de clase $\mathrm{K}_{x}$ si además $f(\mathrm{~s}) \rightarrow \infty$ cuando $\mathrm{s} \rightarrow$ $x$.

Definición 6.- Se dice que una función $f(x)$ es positiva (negativa) definida sobre un subconjunto $\Omega^{\prime}$, de $\Omega$, si $f(x): \Omega \rightarrow \mathbb{R}_{+}\left(\mathbb{R}_{\text { }}\right)$ para todo $x \in \Omega^{\prime}$. 
Capítulo Segundo

\section{Control Robusto de Reactores Químicos Basado en Estimación Discreta de Tasa de Reacción}


Capitulo Segundo

Los resultados de este capítulo fueron complementados de aquellos publicados en los artículos mencionados en el prefacio. Deseo agradecer la colaboración de los coautores involucrados en ellos. 


\section{Control Robusto de Procesos Químicos Basado en Estimación Discreta de Tasa de Reacción}

En este capitulo se tratará con el control de plantas no lineales de primer orden con errores en modelo. Se parte de suposiciones prácticas para llegar a un algoritmo de control robusto basado en estimación de incertidumbres. Las incertidumbres son estimadas mediante un esquema discreto. De este modo, se dispone de un estimado de las incertidumbres y de medidas en línea para obtener una aproximación de los términos no lineales desconocidos. Así pues, éstos pueden ser contrarrestados y la dinámica deseada puede ser inducida. En la primer sección, se estudia la estabilización de un RCTA no isotérmico mientras que en la segunda se presenta una implementación experimental de regulación robusta en efluente de un reactor biológico para tratamiento de aguas residuales a escala piloto.

\subsection{Estabilización robusta de un reactor de alimentación continua}

Consideremos el RCTA representado por el esquema de la Figura 2 con estados $x=$ $\left(x_{1}, x_{2}\right) \in \mathbb{R}^{\mathrm{m}-1}$, donde $x_{1}$ es el vector de concentraciones y $x_{2}$ es la temperatura del reactor. La entrada de control, $u$, es la temperatura de la chaqueta necesaria para estabilizar al RCTA. Por medio de principios fundamentales, y sin considerar la dinámica de la chaqueta, el modelo del reactor tomará la forma del sistema (1) y estará dado por

$$
\begin{aligned}
& \dot{x}_{1}=L\left(x_{1}\right)+R(x) \\
& \dot{x}_{2}=L\left(x_{2}\right)+\beta(x) R(x)+\gamma u
\end{aligned}
$$

donde $\mathrm{L}\left(x_{2}\right)=\theta\left(x_{2, \mathrm{e}}-x_{2}\right)+\gamma x_{2}$ y $\mathrm{L}\left(x_{1}\right)=\theta\left(x_{1, \mathrm{e}}-x_{1}\right), \theta=\mathrm{F} / \mathrm{V}$ es el inverso de tiempo de residencia ( $\mathrm{F}$ es el caudal de alimentación y $\mathrm{V}$ el volumen del reactor), $\gamma$ es un coeficiente conocido. $\beta(x)$ es la entalpía asociada a la tasa de conversión ${ }^{3} R(x)$ y $x_{\mathrm{e}}=\left(x_{1, e}, x_{2, \mathrm{e}}\right)$ es un vector que representa la alimentación al reactor.

Antes de abordar el problema de control recordemos brevemente las principales propiedades de la tasa de conversión química, De acuerdo con el conocimiento previo sobre reactores químicos se sabe que $R(x)$ tiene las siguientes propiedades [2]: P.1) $R\left(x_{1}, 0\right) \equiv 0$ y P.2) $R(x) \leq R\left(x_{1} ; x_{2}^{*}\right) \beta(x) \leq \beta_{1}<x$, donde $x_{2}^{*}$ es una constante. Respecto a (P.1), es bien sabido que no existe reacción a cero grados, o bien como se verá en la sección 2.4 , el consumo de sustrato en un reactor biológico es cero si la concentracion de sustrato es cero. Referente a (P.2) significa que la tasa de reacción está acotada. Adicionalmente recordemos que el reactor isotérmico $\dot{x}_{1}=\mathrm{L}\left(x_{1}\right)+R\left(x_{1} ; x_{2}^{*}\right)$ tiene un único punto de equilibrio estable [2]. Por lo tanto, el sistema (2) es estable si el subsistema (2.2) es estable [13].

\footnotetext{
${ }^{3}$ Fntiéndase por conversión la transformación quimica de una sustancia en otra. Así, una reacción química es la transformación de reactante(s) en producto(s). O bien, el consumo de sustrato es las tranformación de alimento ingerido por microorganismos en desechos producidos en la digestión (i.e, $\mathrm{CO}_{2} \circ \mathrm{CH}_{4}$ )
} 
Capitulo Segundo

Asumamos lo siguiente:

H.2.1) $x_{2}$ está disponible mediante mediciones en línea.

H.2.2) $L_{1}(x)$ y $L_{2}(x)$ son funciones conocidas, (lo cual implica que $\theta$ y $\gamma$ son parámetros conocidos).

H.2.3) El término, $R(x)$, es desconocido pero satisface las propiedades (P.1) y (P.2).

Las justificaciones a cada una de las supocisiones anteriores se harán cuando sea pertinente.

Una forma de estabilizar la temperatura del reactor (2) consiste en diseñar una ley de control por retroalimentación de estados

$$
u_{I}=\frac{1}{\gamma}\left[-\theta\left(x_{2, e}-x_{2}\right)-\beta(x) R(x)+\gamma x_{2}-K\left(x_{2}-x_{2}^{*}\right)\right]
$$

donde $\mathrm{K}>0$ y el subindice $I$ indica ideal. La reatroalimentación de estados (3) es un estabilizador global (en el subconjunto $M \subset \mathbb{R}_{+}^{m^{+1}}$ ) para el sistema (2). Se dice que la retroalimentación (3) es ideal porque lleva a cabo la cancelación exacta de las no linealidades. En consecuencia, para su aplicación se requiere el conocimiento preciso del modelo del RCTA. Empero, de acuerdo con (H.2.3), el modelo dinámico del reactor es incierto, por ello se debe considerar alguna aproximación.

Sean $\beta^{\mathrm{e}}(x)$ y $R^{\mathrm{e}}(x)$ estimados de $\beta(x)$ y $R(x)$, respectivamente. Asumamos que $\beta^{\mathrm{e}}(x)$ y $R^{\mathrm{e}}(x)$ satisfacen las propiedades (P.1) y (P.2). Entonces alternativamente a (3), uno puede usar la siguiente retroalimentación

$$
u=\frac{1}{\gamma}\left[-\theta\left(x_{2, e}-x_{2}\right)-\beta^{e}(x) R^{e}(x)+\gamma x_{2}-K\left(x_{2}-x_{2}^{*}\right)\right]
$$

bajo la retroalimentación (4), las trayectorias del sistema (2) están acotadas para todo $t \geq 0$ [13]. El hecho de que las trayectorias de (2) estén acotadas permite el uso de estabilización mediante alta ganancia para acrecentar la región de estabilidad del sistema a lazo cerrado. Más aún, es posible lograr la estabilización de (1) mediante la retroalimentación de alta ganacia (4), a pesar de no disponer de información alguna sobre el estimado de la tasa de conversión (y por consecuencia de la entalpía, $\beta(x)$ ).

Es necesario recordar la siguiente noción [14]. Se dice que el sistema a lazo cerrado (2),(4) es estabilizable prácticamente si para algún conjunto compacto $\Sigma \subset M$ que contenga a la referencia $x^{*}$, existe una ley de control (estabilizador práctico) la cual hace de este conjunto el atractor estable del sistema en una vecindad $\delta(\Sigma) \subset \mathrm{M}$ de $\Sigma$, es decir, que la trayectoria del sistema que inicie dentro de $\delta(\Sigma)$ converge a $\Sigma$.

Proposición 2.1: La retroalimentación de alta ganancia (4) es un estabilizador práctico del RCTA (2) en el subconjunto $M$. 
Control Robusto de Reactores Químicos Basado en Estimación Discreta de Tasa de Reacción.

Prueba: Sea $z=x_{2}-x_{2}^{*}$. Entonces, bajo la ley de control (4), la ecuación dinámica de la temperatura del RCTA dada por el subsistema (2.2) puede escribirse como sigue

$$
\dot{z}=-K z+\phi\left(x_{1}, z\right)
$$

donde $\phi\left(x_{1}, z\right)=\beta(x) R(x)-\beta^{\mathrm{e}}(x) R^{\mathrm{e}}(x)$. Considerando las propiedades (P.1) y (P.2) e integrando la ecuación (5) se obtiene la siguiente desigualdad

$$
|z| \leq|z(0)| \exp (-K t)+\alpha_{1} \exp (-K t) \int_{0}^{t} \exp (K \sigma) d \sigma
$$

donde $\alpha_{1} \geq\left|\phi\left(x_{1}(\mathrm{t}), \mathrm{z}(\mathrm{t})\right)\right|$. Entonces, $|\mathrm{z}(\mathrm{t})| \leq\left[|\mathrm{z}(0)|-\alpha_{1} / \mathrm{K}\right] \exp (-\mathrm{Kt})+\alpha_{1} / \mathrm{K}$ lo cual implica que $|z(t)|$ está uniformemente acotada. También, $\lim _{t \rightarrow \infty}|z(t)| \leq \alpha_{1} / K$. Dado que las trayectorias de $x_{1}$ están acotadas y son asintóticamente estables para el RCTA isotérmico, la continuidad de la solución implica que las trayectorias a lazo cerrado convergen a una bola $\mathscr{B}(\mathrm{r}(1 / \mathrm{K}))$ centrada en $x^{*}$ y con radio $\mathrm{r}(1 / \mathrm{K})$ del orden de $\alpha_{1} / \mathrm{K}$. De este modo, dado un conjunto compacto arbitrario $\Sigma \subset \mathrm{M}$ que contenga a $x^{*}$, se puede elegir un valor del parámetro de control $\mathrm{K}$, suficientemente grande, tal que $\not(\mathrm{r}(1 / \mathrm{K})) \subseteq \Sigma$. Luego, el controlador (4) es un estabilizador práctico del sistema (2).

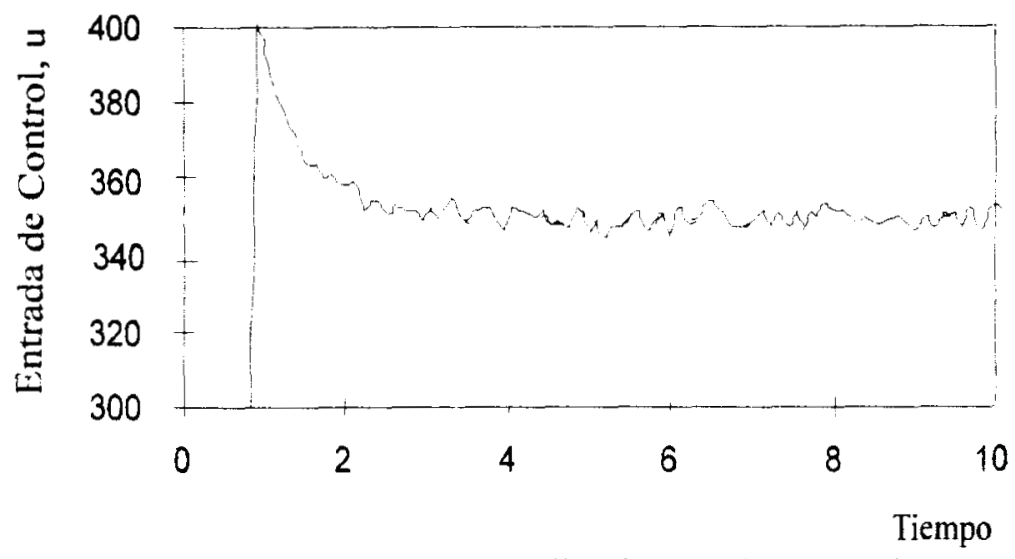

Figura 3. Desempeño del estabilizador práctico (4) en la estabilización del RCTA en $x=(0.5,400)$.

Ejemplo 2.2: Supóngase que no se dispone de información alguna acerca de la tasa de conversión, es decir $\beta^{\mathrm{e}}(x) R^{\mathrm{e}}(x) \equiv 0$ para todo $\mathrm{t} \geq 0$. Considere el RCTA del ejemplo 1.1 (con los mismo valores de parámetros así como orden en la tasa de reacción). De acuerdo con el resultado anterior, el punto de equilibrio inestable $(0.5,400)$ del RCTA puede ser estabilizado prácticamente mediante el estabilizador práctico de alta ganancia (4). En la Figura 3 se muestra el desmpeño de la ley de control (4). Si bien, el reactor es estabilizado, el desempeño obtenido no es aceptable ya que despliega un comportamiento castañeante.

El ejemplo anterior muestra que errores en la estabilización del RCTA (del orden de 
$\mathrm{l} / \mathrm{K}$ ) pueden ser obtenidos por medio de la retroalimentación de alta ganancia (4). A pesar de que no se disponga de información alguna sobre las no linealidades del proceso. Pero, como puede verse en la Figura 3, el controlador de alta ganancia presenta saturación en las acciones de control y ofrece un desempeño castañeante, caracterizado por 'nerviosimo en la entrada de control'. Dicho de otra manera, la alta ganancia ofrece cierto grado de robustez ante errores de modelado, pero el costo a pagar son grandes acciones de control, lo que redunda en un pobre desempeño.

\subsubsection{Un esquema robusto basado en diferencias finitas}

En los siguientes párrafos se detallará el estabilizador práctico propuesto. Como ya se ha dicho, la idea básica consiste en aglutinar las incertidumbres en una función no lineal, la cual puede ser estimada a partir de mediciones en línea e información disponible sobre la acción de control próxima pasada.

Sea $\Theta\left(x_{1}, x_{2}\right)=\beta(x) R(x)$. Entonces, la ecuación dinámica del subsistema (2.2) en el RCTA (2) puede ser reescrita como sigue

$$
\dot{x}_{2}=L\left(x_{2}\right)-\Theta\left(x_{1}, x_{2}\right)+\gamma u
$$

donde $\mathrm{L}\left(x_{2}\right)=\theta\left(x_{2, \mathrm{e}}-x_{2}\right)-\gamma x_{2}$ es la parte lineal del RCTA. En principio uno podría estimar $\Theta\left(x_{1}, x_{2}\right)$ a partir de la solución de $\Theta\left(x_{1}, x_{2}\right)=\dot{x}_{2}-\mathrm{L}\left(x_{2}\right)-\gamma u$. No obstante para ello se requiere conocer la derivada $\dot{x}_{2}$. Sin embargo, $\dot{x}_{2}$ puede ser estimada utilizando diferencias finitas centrales en la $k$-ésima medida. Tomemos a $\Delta \mathrm{T}>0$ como la tasa de muestreo y consideremos la siguiente ley de control

$$
\begin{aligned}
u & =\frac{1}{\gamma}\left[-L\left(x_{2}\right)-\Theta_{k}^{e}\left(x_{1}, x_{2}\right)-K\left(x_{2}-x_{2}^{*}\right)\right] \\
\Theta_{k}^{e}\left(x_{1}, x_{2}\right) & =\frac{1}{\Delta T}\left(x_{2, k}-x_{2, k-1}\right)-L\left(x_{2, k}\right)-\gamma u_{k}
\end{aligned}
$$

donde $\Theta_{k}^{\mathrm{e}}\left(x_{1}, x_{2}\right)$ es un estimado en $\mathrm{t}_{\mathrm{k}}$ de $\Theta\left(x_{1}, x_{2}\right), x_{2 . \mathrm{k}}=x\left(\mathrm{t}_{\mathrm{k}}\right)$ y $u_{\mathrm{k}}=u\left(\mathrm{t}_{\mathrm{k}}\right)$. Nótese que cuando $\Delta \mathrm{T} \rightarrow 0$, el estimado $\Theta_{k}^{\mathrm{e}}\left(x_{1}, x_{2}\right) \rightarrow \Theta\left(x_{1}, x_{2}\right)$. Si $\Theta_{\mathrm{k}}^{\mathrm{e}}\left(x_{1}, x_{2}\right)=\Theta\left(x_{1}, x_{2}\right)$, luego $u_{\mathrm{k}}=u, \mathrm{~L}\left(x_{2, \mathrm{k}}\right)=\mathrm{L}\left(x_{2}\right)$ y la ecuación (8.1) estará dada por $u=\left[-\mathrm{L}\left(x_{2}\right)-\dot{x}_{2}+\mathrm{L}\left(x_{2}\right)+\gamma u-\mathrm{K}\left(x_{2}-x_{2}^{*}\right)\right] / \gamma$, de donde se obtiene la igualdad $\dot{x}_{2}=-\mathrm{K}\left(x_{2}-x_{2}^{*}\right)$. En consecuencia, en el limite cuando $\Delta \mathrm{T} \rightarrow 0$, se recupera el comportamiento de la ley de control ideal (3). Por supuesto, el caso de $\Delta \mathrm{T}=0$ no puede ser implementado porque el controlador estaría mal condicionado.

Aqui son pertinentes un par de observaciones. (i) La ley de control (8.1) puede ser interpretada como una perturbación en $\Delta \mathrm{T}$ del control ideal (3) y, (ii) obviamente, el controlador propuesto consiste en la ley de control de tipo linealizante (8.1) y el compensador dinámico de incertidumbres (8.2). 
Proposición 2.3: El estimador de incertidumbres (8.2) es internamente estable, es decir $\Theta_{k}^{e}\left(x_{1}, x_{2}\right)$ está acotada para todo $\mathrm{k} \geq 1$.

Prueba: Sea $\mathrm{V}=-\mathrm{K}\left(x_{2}-x_{2}^{*}\right)$. Se tienen las siguientes igualdades: $\Theta_{\mathrm{k}}\left(x_{1}, x_{2}\right)=\dot{x}_{2, \mathrm{k}}$ $\mathrm{L}_{\mathrm{k}}\left(x_{2}\right)-\gamma \boldsymbol{u}_{\mathrm{k}}, \gamma \boldsymbol{u}_{\mathrm{k}}=-\mathrm{L}_{\mathrm{k}}\left(x_{2}\right)-\Theta_{\mathrm{k} \cdot \mathrm{l}}^{\mathrm{e}}\left(x_{1}, x_{2}\right)+\mathrm{V}_{\mathrm{k}} \mathrm{y}\left(x_{2, \mathrm{k}}-x_{2, \mathrm{k}-1}\right) / \Delta \mathrm{T}=\dot{x}_{2, \mathrm{k}}+a \Delta \mathrm{T}$, para algún número positivo $a$. Combinándolas, se obtiene $\Theta_{\mathrm{k}}^{\mathrm{e}}\left(x_{1}, x_{2}\right)=\dot{x}_{2, \mathrm{k}}-\mathrm{V}_{\mathrm{k}}+\Theta_{\mathrm{k}-1}^{\mathrm{e}}\left(x_{1}, x_{2}\right)+a \Delta \mathrm{T}$. Por otro lado $\dot{x}_{2, \mathrm{k}}=\mathrm{V}_{\mathrm{k}}+\left[\Theta_{\mathrm{k}}\left(x_{1}, x_{2}\right)-\Theta_{\mathrm{k}}^{\mathrm{e}}\left(x_{1}, x_{2}\right)\right]$. Por lo tanto

$$
\theta_{k}^{e}\left(x_{1}, x_{2}\right)=\frac{\theta_{k}\left(x_{1}, x_{2}\right)+\theta_{k-1}^{e}\left(x_{1}, x_{2}\right)}{2}+a \Delta T
$$

De acuerdo con la propiedad (P.2) $\Theta_{\mathrm{k}}\left(x_{1}, x_{2}\right)$ está acotada para todo $\mathrm{k} \geq 1$. Dado que la ecuación (9) es un mapeo estable (con una tasa de decaimiento de 0.5 ) más un término acotado, entonces $\Theta_{k}^{e}\left(x_{1}, x_{2}\right)$ está acotada para todo $k \geq 1$. $\square$

Nótese que el estimado (9) es el promedio aritmético entre el valor actual de la incertidumbre y el valor inmediato anterior del estimado más un término que surge de la aproximación de primer orden, en diferencias finitas centrales, a la derivada de la temperatura. Entoces, en el límite cuando $\Delta \mathrm{T} \rightarrow 0, \Theta_{\mathrm{k}-1}^{\mathrm{e}}\left(x_{1}, x_{2}\right) \rightarrow \Theta_{\mathrm{k}}^{\mathrm{e}}\left(x_{1}, x_{2}\right)$.

Teorema 2.4: El controlador (8) es un estabilizador práctico para el subsistema (2.2).

Prueba: Sea $\mathrm{z}=x_{2}-x_{2}^{*}$. A lazo cerrado $\dot{\mathrm{z}}_{2}=-\mathrm{Kz}+\left[\Theta_{\mathrm{k}}\left(x_{1}, \mathrm{z}\right)-\Theta_{k}^{\mathrm{e}}\left(x_{1}, \mathrm{z}\right)\right]$, para el intervalo $t \in\left(t_{k}, t_{k+1}\right]=\left(t_{k}, t_{k}+\Delta T\right]$. Además, dado que el controlador (8) es estable (proposición 2.2), $\left|\Theta_{\mathrm{k}}\left(x_{1}, \mathrm{z}\right)-\Theta_{\mathrm{k}}^{\mathrm{e}}\left(x_{1}, z\right)\right|=\left|\dot{x}_{2}-\mathrm{L}\left(x_{2}\right)+\gamma\left(u_{\mathrm{k}}-u\right)-\left[x_{2, \mathrm{k}}-x_{2, \mathrm{k}-\mathrm{l}}\right] / \Delta \mathrm{T}+\mathrm{L}\left(x_{2, \mathrm{k}}\right)\right| \leq$ $a \Delta \mathrm{T}+\left|\gamma\left(u_{\mathrm{k}}-u\right)\right|+\left|\mathrm{L}\left(x_{2}\right)-\mathrm{L}\left(x_{2, \mathrm{k}}\right)\right|=\left(a+a_{1}+a_{2}\right) \Delta \mathrm{T}$, donde $a_{1}$ y $a_{2}$ son números positivos finitos. Por otra parte, de la integración se obtiene: $\{z(t) \mid=[|z(0)|-a \Delta T / K] \exp (-\mathrm{Kt})+a \Delta / \mathrm{K}$, y consecuentemente $|z|_{\infty} \leq a \Delta \mathrm{T} / \mathrm{K}$. Al igual que en la proposición 2.1, el resultado para $|z|_{\infty}$ implica que la trayectoria del sistema a lazo cerrado converge a una bola $\$(r(\Delta \mathrm{T} / \mathrm{K}))$. Entonces, se puede concluir que tomando un parámetros de control $\Delta \mathrm{T} / \mathrm{K}$ suficientemente pequeño, la trayectoria puede ser llevada a una vecindad arbitrariamente pequeña del punto de equilibrio $x_{2}^{*}$.

Corolario 2.5: El controlador (8) es un estabilizador práctico del RCTA (2).

Ejemplo 2.6: Consideremos el RCTA utilizado en el ejemplo 2.1. Pero ahora será estabilizado por medio del controlador (8). Tomemos $\Delta \mathrm{T}=0.01$, lo que significa una frecuencia de muestreo de $100 \mathrm{~Hz}$. El parámetro de control es elegido $\mathrm{K}=1.0$. En la Figura 4 se muestra el desempeño del controlador (8). Se puede observar en la Fig. 4 a que el valor estimado de la incertidumbre converge suavemente a su valor real de la generación de calor en estado estacionario $\left(\Theta\left(x_{1}, x_{2}\right)=x_{1}^{*} \beta \xi_{0} \exp \left(-E a / R x_{2}^{*}\right)=100.0\right)$. Debido al hecho que se dispone de un buen estimado del término incierto, las acciones de control requeridas para lograr la estabilización son suaves (ver Fig. 4.b). Se incluyó saturación en el controlador (8), sin embargo las acciones nunca se saturaron, así que es de esperarse que no se presente degradación del control. Comparemos las acciones de control logradas por medio de alta 
Capitulo Segundo

ganancia (Fig. 3). Con el control propuesto se obtiene un mucho mejor desempeño.
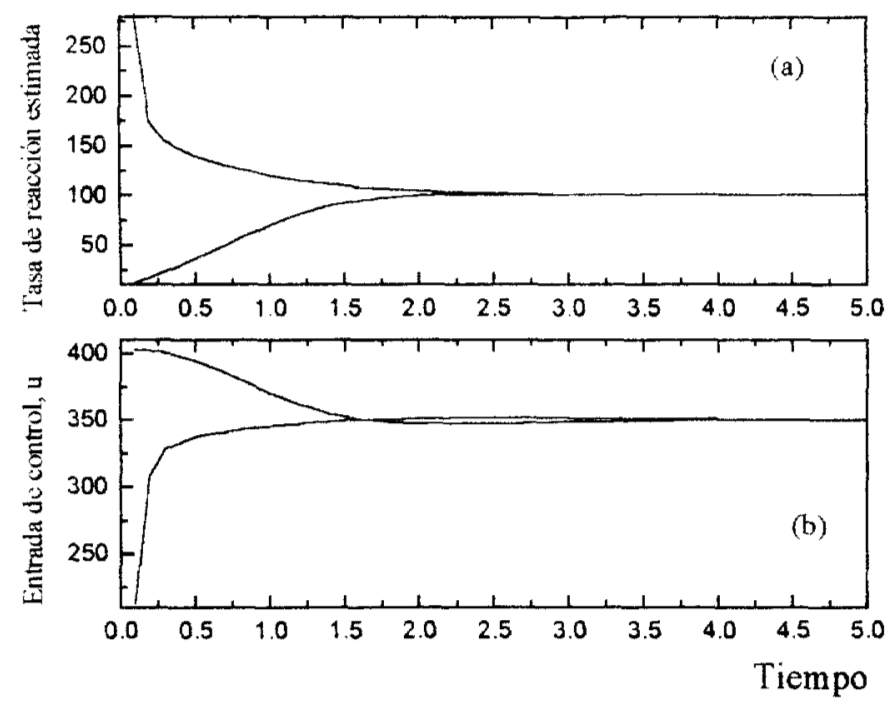

Figura 4. Desempeño del Controlador (8) para diferentes condiciones iniciales.

\subsection{Reactor continuo de polimerización (un RCTA no isotérmico)}

Los RCTA no isotérmicos son muy comunes en la elaboración de productos de alto valor agregado. No es frecuente que la agitación del reactor contribuya de forma importante al balance de energía si se le compara con el flujo de calor entálpico (es decir, las variaciones de entalpía liberada (o absorbida) por remoción (o adición) premeditada de calor). Por lo tanto, se puede ignorar el término correspondiente al trabajo en el balance de energía. Este es el caso de los reactores de polimerización cuya liberación entálpica es muy grande. Cabe mencionar que el control robusto en reactores de polimerización es sumamente importante, y a que los materiales elaborados (polímeros) son de alto valor agregado y, adicionalmente, su comportamiento cinético no es sencillo. De hecho, las expresiones para la cinética, si bien, se sabe que satisfacen las propiedades (P.1) y (P.2), no son conocidas con precisión. Además, todavía se encuentran en estudio muchos fenómenos asociados con la polimerización (como el de transporte de masa), y aún en dichos estudios, es conveniente implementar regulación robusta de temperatura puesto que variaciones en temperatura pueden ser fatales en el estudio cinético (por ejemplo, en el caso de polimerización en emulsión).

Así pues, consideremos un RCTA no isotérmico donde la polimerización de metilmetacrilato vía radicales libres se lleva a cabo con azo-bis-isobutilnitrilo como iniciador y tolueno como disolvente. La reacción es exotérmica y una chaqueta de enfriamiento permite la remoción de calor. De acuerdo con las suposiciones hechas en [15], el modelo del reactor puede ser escrito de la siguiente forma 


$$
\begin{aligned}
& \dot{x}_{1,1}=-\left[z_{p} \exp \left(-E a_{p} / R x_{2}\right)+z_{f m} \exp \left(-E a_{f m} / R x_{2}\right)\right] x_{2} P_{0}\left(x_{1,2} x_{2}\right)+\theta\left(x_{1,1, e}-x_{1,1}\right) \\
& \dot{x}_{1,2}=-z_{I} \exp \left(-E a / R x_{2}\right) x_{1,2}+\theta_{I}\left(x_{1,2, e}-x_{1,2}\right) \\
& \dot{x}_{2}=\beta_{p} z_{p} \exp \left(-E a_{p} / R x_{2}\right) x_{1,1} P_{0}\left(x_{1,2} x_{2}\right)+\theta\left(x_{2, e}-x_{2}\right)-\gamma\left(x_{2}-u\right)
\end{aligned}
$$

donde $\theta_{\mathrm{I}}=\mathrm{F}_{\mathrm{I}} / \mathrm{V}, \mathrm{F}_{\mathrm{I}}$ es el caudal de alimentación de iniciador. $x_{1}=\left(x_{1,1}, x_{1,2}\right) \in \mathbf{R}_{+}^{2}$ es el vector de composiciones (monómero e iniciador, respectivamente). Adicionalmente, una expresión para la tasa de polimerización $P_{0}\left(x_{1,2}, x_{2}\right)$ está dada por

$$
P_{0}\left(x_{1,2}, x_{2}\right)=\left[\frac{2 f^{*} x_{1,1} z_{I} \exp \left(-E a_{I} / R x_{2}\right)}{z_{T_{d}} \exp \left(-E a_{T_{d}} / R x_{2}\right)+z_{T_{c}} \exp \left(-E_{T_{c}} / R x_{2}\right)}\right]^{0.5}
$$

entonces, el problema de estabilización del RCTA no isotérmico (10) consiste en diseñar una ley de control tal que $\dot{x}_{2}=\theta\left(x_{2, e}-x_{2}\right)+\beta(x) R(x)-\gamma\left(x_{2}-u\right)$ sea estable (lo cual también puede ser interpretado como regulación de temperatura).

Es necesario hacer algunos comentarios pertinentes respecto a las hipótesis planteadas en la sección anterior. (H.2.1) es una suposición realista. En la actualidad exiten diversos sensores y procesadores que permiten medir temperatura hasta a $1000 \mathrm{~Hz}$.

La hipótesis (H.2.2) es la más fuerte. Como ya ha sido discutido, existe incertidumbre asociada al modelo. Además, pueden existir variaciones en tiempo de los parámetros involucrados (como en el coeficiente de transferencia de calor), empero se asumirá que son conocidos. Sin embargo, tal suposición será relajada en los siguientes capitulos. Dado que aquí se demostrará convergencia exponencial, por continuidad, se puede esperar cierto grado de robustez ante pequeñas variaciones paramétricas.

En cuanto al tercer supuesto, (H.2.3) es una hipótesis práctica.

Adicionalmente, se tienen las siguientes afirmaciones: (a) El hecho que el sistema (10) satisface la propiedad (P.1) es directamente comprobable, puesto que la tasa de reacción es de tipo Arrhenius. Por otra parte, en lo que concierne a (P.2) de la ecuación (11) se tiene que $\mathrm{P}_{0}\left(x_{1,2}, x_{2}\right) \leq \mathrm{P}_{0}^{\max }=\left[2 f^{*} x_{1,2, \mathrm{e}} \mathrm{z}_{1} /\left(\mathrm{z}_{\mathrm{Td}}+\mathrm{z}_{\mathrm{Tc}}\right)\right]$ de donde

$$
\left[z_{p} \exp \left(-\frac{E a_{p}}{R x_{2}}\right)+z_{f m} \exp \left(-\frac{E a_{f m}}{R x_{2}}\right)\right] x_{1}, 1 P_{0}\left(x_{1,2}, x_{2}\right) \leq\left(z_{p}+z_{f m}\right) x_{1, e} P_{0}^{\max }
$$

entonces, $z_{1} \exp \left(-E a_{1} / R x_{2}\right) x_{2} \leq z_{1} x_{2, e}$ (b) El RCTA isotérmico es globalmente asintóticamente estable. Esto es fácil de probar, es suficiente observar que para una temperatura dada $x_{2}=x_{2}^{*}$, la dinámica del iniciador estará dada por la siguiente expresión: $\dot{x}_{1,2}=-\left(\lambda^{*}+\theta\right) x_{1,2}+\theta_{I} x_{1,2 . e}$ donde $\lambda^{*}=z_{1} \exp \left(-E a_{1} / R x_{2}^{*}\right)$. 
Capitulo Segundo

Como una consecuencia de las afirmaciones anteriores, se deduce que el RCTA de polimerización es estabilizable por medio del control robusto con estimación de incertidumbres dado por ec. (8) (Corolario 2.4). Puede notarse que para la implementación de (8) no se requieren mediciones de concentración alguna. En la Figura 5 se muestra la dinámica de la temperatura y el desempeño del controlador (8). Para las simulaciones se utilizaron los parámetros cinéticos reportados en [15]
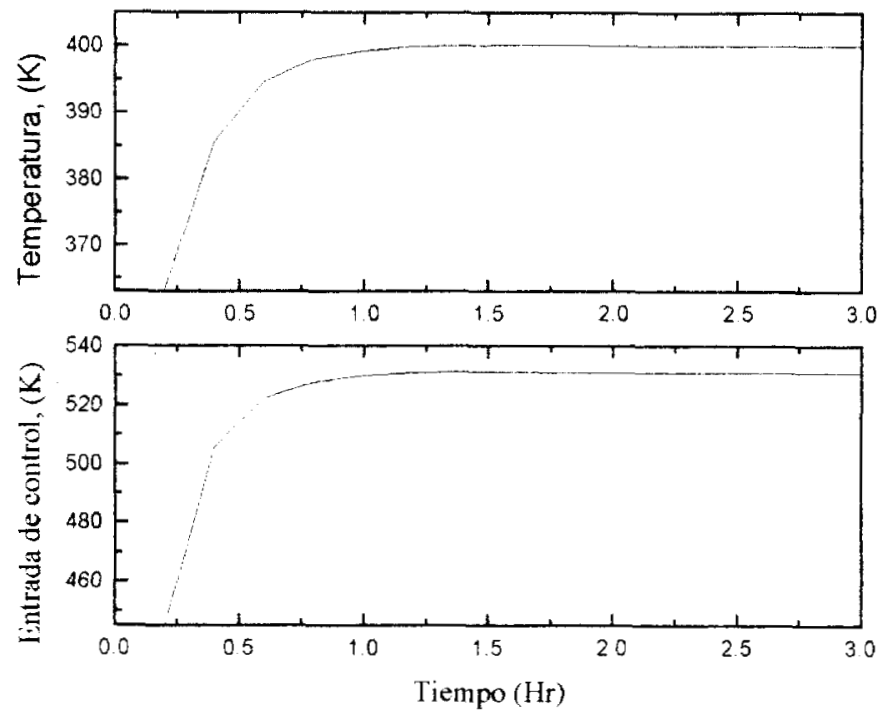

Figura 5. Desempeño del controlador (8) en la estabilización del reactor de polimerización (10).

\subsection{Regulación robusta de sustrato en un digestor anaerobio}

Ahora, consideremos otra clase de proceso químico. Un RCTA estable de gran interés cuyo modelo tiene la forma del sistema (1). La digestión anaerobia es un proceso ampliamente usado para el tratamiento de aguas residuales. En éste se promueve la degradación de sustancias orgánicas a $\mathrm{CO}_{2}$ y $\mathrm{CH}_{4}$ mediante microorganismos (por ello se le conoce como biorreactor). Este proceso comprende dos etapas. En la primera, los compuestos orgánicos son fermentados a ácidos grasos volátiles y $\mathrm{CO}_{2}$ por un grupo de bacterias acidogénicas. En la segunda, los ácidos grasos volátiles son convertidos $\mathrm{CO}_{2}$ y $\mathrm{CH}_{4}$ por bacterias metanogénicas.

Debido a la complejidad e importancia del proceso anaerobio, la atención de diversas áreas se ha enfocado al mejoramiento de su desempeño, el control de procesos es una de ellas. Diversas estrategias han sido propuestas para controlar la operación de la digestión anaerobia (ver [4] y las referencias citadas ahí). Los esquemas adaptativos han mostrado ser apropiados para la regulación de salida (concentración de sustrato) en este proceso químico. Sin embargo, como veremos en una comparación realizada en los siguientes párrafos, los algoritmos de 
Control Robusto de Reactores Quimicos Basado en Estimación Discreta de Tasa de Reacción.

control adaptable hasta ahora desarrollados requieren demasiadas mediciones, especificamente medidas de las concentraciones tanto de gas producido por las bacterias (biogas) como de los microorganismos mismos (biomasa).

Asumamos la descripción y modelamiento dinámico más aceptado para un digestor anaerobio [16]. Mediante principios fundamentales (balance de masa), en ambas etapas del biorreactor, se puede obtener el comportamiento dinámico más simple de sustrato y biomasa como sigue

$$
\begin{aligned}
& \dot{x}_{1,1}=\mu_{1}(x) x_{1,1}-x_{1,1} u \\
& \dot{x}_{1,2}=\mu_{2}(x) x_{1,2}-x_{1,2} u \\
& \dot{x}_{2,1}=-k_{1} \mu_{1}(x)+\left(x_{2,1, e}-x_{2,1}\right) u \\
& \dot{x}_{2,2}=-k_{2} \mu_{2}(x)+k_{3} \mu_{1}(x) x_{1,1}-x_{2,2} u
\end{aligned}
$$

donde $x_{1}=\left(x_{1,1}, x_{1,2}\right)^{\mathrm{T}} \in \mathbf{R}^{2}$ es el vector (concentración total de biomasa) cuyos elementos son las concentraciones de bacterias acidogénicas y metanogénicas, respectivamente. $x_{2}=$ $\left(x_{2,1}, x_{2,2}\right)^{\mathrm{T}}$ es el vector (concentración total de sustrato) cuyos elementos son las concentraciones de sustrato en la primer y segunda etapa, respectivamente. $\mu_{\mathrm{i}}(x), \mathrm{i}=1,2$, se conoce como tasa específica de crecimiento de biomasa. Las $\mathrm{k}_{\mathrm{j}}^{\mathrm{s}}$ son coeficientes de crecimiento de biomasa. Adicionalmente, la producción de biogas está dada por

$$
\mathrm{Q}_{1}=\mathrm{k}_{4} \mu_{2}(x) x_{2} \quad \text { y } \quad \mathrm{Q}_{2}=\mathrm{k}_{5} \mu_{1}(x) x_{1}+\mathrm{k}_{6} \mu_{2}(x) x_{2}
$$

A pesar que el sistema (13) es la representación más simple del modelo del digestor, retiene las principales características de su comportamiento dinámico [13]. Nótese que el modelo (13), tiene la forma del sistema (1). Así pues, cuando la digestión anaerobia es usada en tratamiento de aguas residuales, el objetivo de control consiste en diseñar una ley de control tal que se regule la concentración de sustrato a pesar de errores de modelado, dicho de otra forma, $x_{2} \rightarrow x_{2}^{*}$ cuando $t \rightarrow t^{*}<\infty$.

Consideremos las suposiciones (H.2.1) y (H.2.3) y definamos $x_{2}=x_{1,1}+x_{1,2}$ como la concentración total de sustrato en el digestor. Combinando las ecuaciones dinámicas de sustrato en cada etapa se tiene que la concentración total de sustrato está dada por la siguiente ecuación

$$
\dot{x}_{2}=R(x)+\left(x_{2, e}-x_{2}\right) u
$$

donde $R(x)=-a_{1} \mathrm{Q}_{1}+a_{2} \mathrm{Q}_{2}$ es la tasa de consumo de sustrato en términos de producción de biogas. $Q_{1}$ y $Q_{2}$ están dadas por las expresiones antes mencionadas. Finalmente, los coeficientes $a_{1,2}$, desconocidos, están dados por 
Capitulo Segundo

$$
a_{1}=\frac{1}{k_{4}}\left(k_{2}+\frac{\left(k_{3}-k_{1}\right) k_{6}}{k_{5}}\right), \quad a_{2}=\frac{k_{3}-k_{1}}{k_{5}}
$$

Una vez más es pertinente hacer algunas observaciones importantes. En lo que respecta a las hipótesis se pueden hacer las siguientes afirmaciones: (i) (H.2.1) es práctica. La concentración total de sustrato se puede medir en línea por medio de la demanda química de oxígeno (COD por sus siglas en inglés) [13]. (ii) (H.2.3) es realista. De acuerdo con las ecuaciones (14) y (15), está claro que $R(x)$ depende de las tasas específicas de crecimiento de sustrato $\mu_{1}(x)$ y $\mu_{2}(x)$ las cuales no sólo son función de $x_{1}$ y $x_{2}$, sino también dependen de $\mathrm{pH}$, régimen hidrodinámico, temperatura ambiente, concentración de sustancias inhibidoras, etc. Es por ello, que $\mu_{1}(x)$ y $\mu_{2}(x)$ son funciones desconocidas y por consecuencia $R(x)$ también. (iii) En lo concerniente a las propiedades (P.1) y (P.2), se sabe que la tasa de consumo de sustrato las satisface. Dado que el sustrato es el alimento de los microorganismos, si $x_{2}=0$, la producción de biogas es cero por lo tanto $R\left(x_{1}, 0\right)=0$. Por ejemplo, supongamos que $\mu_{1}(x)$ y $\mu_{2}(x)$ están dadas por la ley de Monod, es decir $\mu(x)=\mu_{\max } x_{2} /\left(\mathrm{K}_{M}+x_{2}\right)$, donde $\mu_{\max }$ es la máxima tasa especifica de crecimiento y $K_{M}$ es una constante de afinidad al sustrato. Entonces, si $x_{2}=0, \mu(x)=0$ consecuentemente $Q_{1}=Q_{2}=0$, por consiguiente $R\left(x_{1}, 0\right)=0$. Finalmente, (iv) La tasa de conversión está acotada. En particular, si asumimos que $\mu_{1,2}(x)$ están dadas por Monod, $R(x)$ está acotada por la máxima tasa específica de crecimiento, $\mu_{\max }$.

\subsubsection{Una implementación experimental}

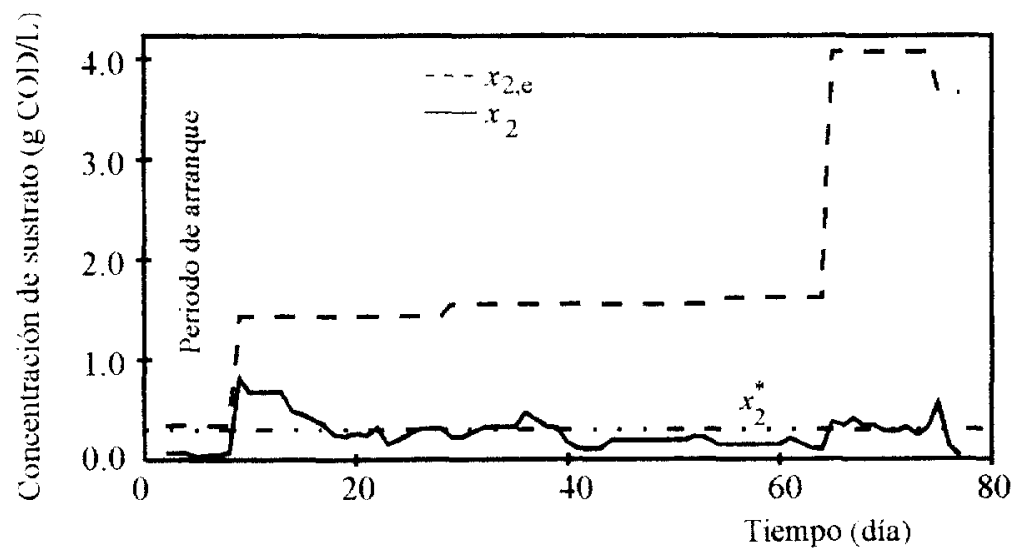

Figura 6. Regulación de sustrato en un digestor anaeronbio a escala piloto

Sea $\mathrm{L}(x)=0, \Theta^{\mathrm{c}}\left(x_{1}, x_{2}\right)=R(x)$ y $\mathrm{K}=1$ con $\Delta \mathrm{T}=1$ dia. La referencia $x_{2}^{*}=0.3 \mathrm{~g}$ $\mathrm{COD} / \mathrm{L}$. La ley de control está dada por : $u_{\mathrm{k}}=\left[-\Theta_{\mathrm{k}}^{\mathrm{e}}\left(x_{1}, x_{2}\right)-\mathrm{K}\left(x_{2, \mathrm{k}}-x_{2, \mathrm{k}}^{*}\right)\right] /\left(\mathrm{s}_{\mathrm{e}, \mathrm{k}}-x_{2, \mathrm{k}}\right)$ donde $\Theta_{\mathrm{k}}^{\mathrm{a}}\left(x_{1}, x_{2}\right)=\left[x_{2, \mathrm{k}}-x_{2, \mathrm{k}-1}\right] / \Delta \mathrm{T}-\left(\mathrm{s}_{\mathrm{e}, \mathrm{k}}-x_{2, \mathrm{k}}\right) u_{\mathrm{k}, \mathrm{l}}$.

Luego, la salida del digestor anaerobio puede regularse mediante el controlador 
basados en estimación discreta de incertidumbres. La implementación se efectuó en un reactor a escala piloto con $2.0 \mathrm{~L}$ de volumen total. El biorreactor fue alimentado con lodos de un reactor a escala media usado para tratamiento de aguas. El sustrato consistió en una solución de lactosa enriquecida. La medición de sustrato fue hecha cada día con métodos conocidos para COD (para mayores detalles ver [4]). En la Figura 6 se muestra la regulación de sustrato en el digestor. El estabilizador práctico (8) permite la regulación de la salida a pesar de los errores de modelado y perturbaciones en la alimentación de sustrato. Cabe señalar que la regulación de sustrato se logra frente a perturbaciones en la alimentación de sustrato más severas [17].

Dochain y Bastin [16], propusieron un esquema adaptativo de control donde se estiman los coeficientes de $R(x)=-a_{1} Q_{1}+a_{2} Q_{2}$ a partir de mediciones de biogas. Para ello se asume que la planta es lineal en los parámetros. Sin embargo, su variación en el tiempo muestra que dichos parámetros no son lineales [17]. De hecho, el esquema de control adaptable tiene las siguientes desventajas: (i) requiere medidas de biogas y (ii) el esquema de estimación de incertidumbres es más complicado. Con el objetivo de comparar ambos esquemas de control (el aquí desarrollado y el propuesto por Dochain y Batin [16]), fue calculada la acción de control resultante a partir de mediciones de biogas (sin que fuese aplicada al digestor). La comparación de ambas entradas de control se muestra en la Figura 7.

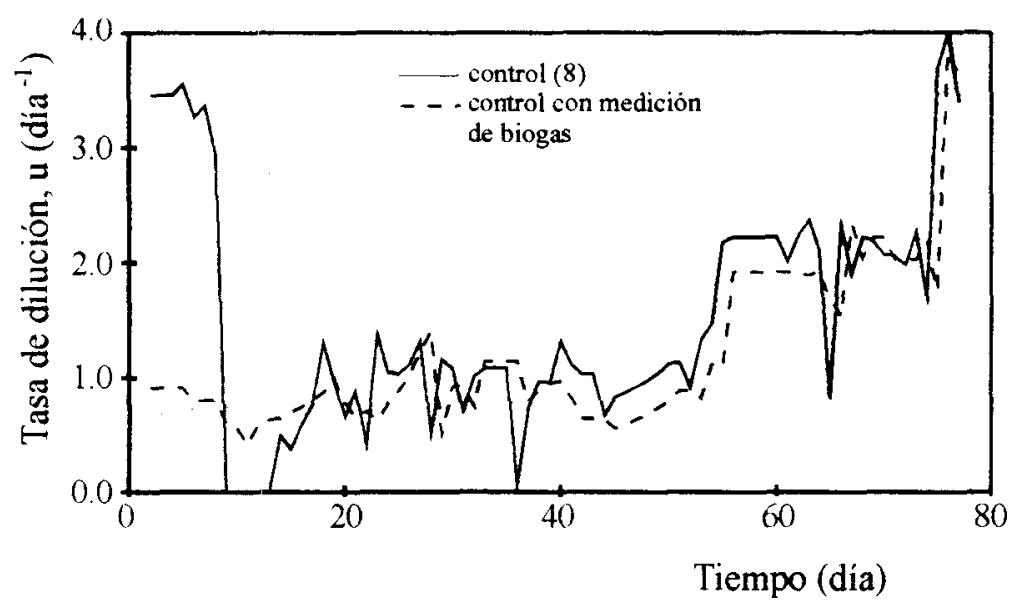

Figura 7. Comparación entre las acciones de control calculadas por ecuación (8) y las reportadas en [16].

Evidentemente, las acciones de control son similares y únicamente en la etapa de arranque el desempeño del controlador (8) es pobre. De acuerdo con los resultados de la sección 2.1, el comportamiento dinámico del control y la regulación de sustrato puede mejorar si se disminuye la frecuencia de muestreo $\Delta T$ o se aumenta la ganancia $K$ (una opción es medir cada 4 o 6 horas en vez de cada 24 ). 
Capitulo Segundo

\subsection{Discusión y observaciones concluyentes}

En esta sección se desarrolló un algoritmo de control robusto ante errores de modelado. El esquema está basado en estimación discreta de incertidumbres y una ley de control de tipo linealizante. Todas las señales internas del controlador son estables. Asi pues, la aproximación permite estabilizar prácticamente un RCTA a pesar de perturbaciones en la alimentación del reactor e incertidumbres en modelo. Además, se observó experimentalmente que el control robusto puede regular la salida en presencia de perturbaciones a la entrada de reactor.

Por un lado, la comparación del controlador propuesto con control de alta ganancia muestra las ventajas del controlador (8). Debido a que el estimador discreto provee a la ley de control linealizante con un estimado del término incierto tiempo a tiempo, las acciones de control son más suaves que aquellas requeridas con el uso de control basado en al ta ganacia. Por otro lado, la comparación del CAR con un esquema previamente reportado ofrece una indubitable evidencia que el controlador (8) presenta desempeño similar pero con menos mediciones, lo cual es una enorme ventaja económica. Por ello, es de esperarse que la operación del controlador diseñado sea más barata que la del control previamente reportado. Luego, la implementación experimental en un reactor a escala piloto muestra la viabilidad del esquema. Más aún, esta implementación hace incuestinable la eventual aplicación práctica del algoritmo a un RCTA en una escala mayor.

No obstante, aquí nada más se trata con plantas de primer orden. $Y$ aunque, mediante argumentos de continuidad, es de esperarse un cierto grado de robustez ante pequeñas variaciones paramétricas en la planta y perturbaciones, sólo se garantiza que el controlador (8) puede contrarrestar errores en modelo. Sin embargo, por una parte, la estimación discreta puede presentar dificultades para la aplicación del controlador (8) a plantas de orden superior. Sobre todo para probar estabilidad a lazo cerrado. Por otra, algunas suposiciones hechas deben ser relajadas (por ejemplo, la concerniente al conocimiento de la parte lineal del modelo).

Por ello, es necesario modificar el algoritmo de tal manera que se vislumbre una generalización. Respecto al esquema de estimación, es conveniente plantear un estimador continuo sin que se pierdan las caracteristicas y propiedades esenciales del algoritmo, y que a su vez, permita relajar, al menos parcialmente, algunas supoisiciones. Resultados en este sentido son discutidos en el siguiente capítulo, donde primero se relajan algunas de las hipótesis y luego se establece un controlador con compensador dinámico continuo. Además, en el siguiente capítulo se interpreta al CAR en términos de control clásico mediante una transformación de dominio de tiempo al de frecuencia. 
Control Robusto de Reactores Quimicos: Un esquema de control para plantas de primer orden

Capítulo Tercero

\section{Control Robusto de Reactores Químicos: \\ Un esquema de control para plantas de primer orden}


Cupitulo Tercero

Los resultados de este capitulo fueron complementados con todos aquellos publicados en los artículos correspondientes y que fueron mencionados en el prefacio. Nuevamente deseo agradecer la colaboración de los coautores involucrados. 


\section{Control Robusto de Reactores Químicos: Un esquema de control para plantas de primer orden}

En este capitulo se trata con el problema de control robusto de plantas de primer orden. Se parte de una ley de control linealizante para obtener un controlador robusto ante errores en modelo, variaciones paramétricas y perturbaciones. Al igual que el controlador basado en estimación discreta de incertidumbres, el algoritmo aquí propuesto comprende dos partes: (a) un compensador de incertidumbres y (b) una ley de tipo linealizante. Recordemos la idea básica: Se aglutinan las incertidumbres en una función no lineal la cual es interpretada como una variable de estado en un sistema de dimensión 2 (sistema extendido). Luego se construye un observador de estados para el sistema extendido. De esta manera una aproximación a la ley de control linealizante es provista de un estimado de las incertidumbres y éstas pueden ser contrarrestadas. Entonces, el algoritmo resulta en un control asintótico robusto (CAR).

Con el fin de realizar una interpretación clásica del controlador resultante, se lleva a cabo una transformación del CAR usando el operador de Laplace. La interpretación en dominio de frecuencia permite entenderle como un controlador PI con estimación dinámica de incertidumbres. La estimación dinámica de incertidumbres se logra por una acción integral al cuadrado, asi que el controlador ha sido llamado PII $^{2}$. Una ganancia de alta frecuencia es el único parámetro a sintonizar para estimar a las perturbaciones de carga dinámica.

\subsection{CAR de plantas de primer orden}

Consideremos el siguiente sistema dinámico

$$
\dot{x}_{2}=f^{*}(x)+f(x, w)+\left\{g^{*}(x)+g(x, w)\right\} u
$$

donde $f^{*}(x)$ y $\mathrm{g}^{*}(x)$ son funciones suaves conocidas (nominales), mientras que $f(x, w)$ y $\mathrm{g}(x, w)$ son funciones suaves desconocidas. Al igual que en el capitulo anterior $x=\left(x_{1}, x_{2}\right)$ es un vector de estados, $x_{1} \in \mathbb{R}_{+}^{\mathrm{m}}$ es un vector de estados acotados no medidos (ver capítulo anterior) y $x_{2} \in \mathbb{R}_{+}$es el estado disponible para retroalimentación por mediciones en línea. $w=w(t)$ es un vector de señales perturbantes y $u \in \mathbf{R}$ es la entrada de control.

Está claro que la ecuación (16) es una forma generalizada para plantas de primer orden. Incluye términos no lineales con incertidumbre, variaciones paramétricas y perturbaciones dinámicas. En principio, si uno es capaz de estabilizar al sistema (16), cualquier reactor que satisfaga las propiedades (P.1)-(P.2) y cuyo modelo tenga la forma de (16) podrá ser estabilizado.

Asumamos lo siguiente:

H.3.1) $\operatorname{sgn}\left(\mathrm{g}^{*}(x)\right)=\operatorname{sgn}(\mathrm{g}(x, w))$. Además, $\operatorname{sgn}\left(\mathrm{g}(x, w)+\mathrm{g}^{*}(x)\right)>0$.

H.3.2) $w$ y $\dot{w}$ son funciones suficientemente suaves en t. $w$ pertenece a un conjunto 
Capitulo Tercero

compacto 0 contenido en un espacio Euclidiano de dimensión adecuada.

H.3.3) Para todo conjunto compacto $屯 \subset \mathbb{R}$, existe una función escalar no negativa localmente Lipschitz $\beta(x, w)$ conocida tal que $\mathrm{g}^{*}(x) \leq \beta(x, w)$ para todo $x \in \mathscr{\ell}, w \in \mathscr{C}$ y $\mathrm{t} \geq$ 0 . Nótese que si la condición anterior se mantiene globalmente, entonces se mantiene sobre todo el conjunto compacto $屯 \subset \mathbb{R}$

Cabe destacar que no se asume conocimiento alguno sobre cotas del término $f(x, w)$. Por otro lado, si $\mathrm{g}(x, w) \leq 0$ para todo $x \in \mathscr{E}, w \in \mathscr{Q}$ y $\mathrm{t} \geq 0$, entonces se puede tomar a $\beta(x, w)=0$. Con respecto a la verificación y justificación de las suposiciones anteriores, éstas se harán cuando resulte necesario.

Ya se ha probado que el control linealizante de alta ganancia puede estabilizar al sistema (16) (ver \$2). También, se ha mencionado la necesidad de un algoritmo de diseño de controladores robustos. Así pues, el problema de control puede ser replanteado como sigue: Dado un conjunto compacto $₫ \subset \mathbb{R}$, el objetivo es diseñar una ley de control tal que lleve todas las trayectorias con condiciones iniciales $x_{0}$ contenidas en a una vecindad del ongen arbitrariamente pequeña (estabilización práctica) a pesar de incertidumbres en modelo, variaciones paramétricas y perturbaciones en la planta.

Sin pérdida de generalidad, consideremos que $x_{1} \in \mathbb{R}$. Definiendo $z=x_{2}-x_{2}^{*}, \delta(z, w)$ $=\mathrm{g}(\mathrm{z}, w)-\beta(\mathrm{z}, w), \delta^{*}(\mathrm{z}, w)=\mathrm{g}^{*}(\mathrm{z})+\beta(\mathrm{z}, w), \Theta(\mathrm{z}, w, u)=f(\mathrm{z}, w)+\delta(\mathrm{z}, w) \boldsymbol{y}$ y $\eta=\Theta(\mathrm{z}, w, u)$, el sistema (16) puede ser reescrito como sigue

$$
\begin{aligned}
\dot{z} & =\eta+f^{*}(z)+\delta^{*}(z, w) u \\
\dot{\eta} & =\Xi(z, \eta, W, U) \\
\Psi_{0} & =0
\end{aligned}
$$

donde $\Xi(\mathrm{z}, \eta, W, U)=\left[\eta+f^{*}(\mathrm{z})+\delta^{*}(\mathrm{z}, w) u+\dot{x}_{1}\right]\{\partial f(\mathrm{z}, w) / \partial \mathrm{z}+\partial \delta(\mathrm{z}, w) / \partial \mathrm{z}\}+[\partial f(\mathrm{z}, w) / \partial w+$ $\hat{C} \delta(\mathrm{z}, w) / \partial w] w+\partial f(\mathrm{z}, w) / \partial \mathrm{t}+[\partial \delta(\mathrm{z}, w) / \partial \mathrm{t}] u+\delta(\mathrm{z}, w) \dot{u} . W=(w, \dot{w})$ y $U=(u, \dot{u}) . \psi(\mathrm{z}, \eta, w, u)=$ $\Theta(z, w, u)-\eta=0$ es una restricción algebraica y $\psi_{0}=\psi(z(0), \eta(0), w(0), u(0))$.

Nótese que el sistema (17) es dinámicamente externamente equivalente al sistema (16). Esto significa que los sistemas (16) y (17) tienen las mismas soluciones siempre que se satisfaga la restricción $\psi_{0}=0$. De hecho, la superficie $\psi(z, \eta, w, u)=0$ es invariante bajo la dinámica del sistema (17), es decir, $\mathrm{d} \psi(\mathrm{z}, \eta, w, u) / \mathrm{dt}=0$ sobre las trayectorias generadas por el sistema (17). Formalmente, se dice que $\psi(\mathrm{z}, \eta, w, u)$ es una primer integral del sistema (17) [18].

Luego, si uno es capaz de estabilizar al sistema (17) (o en su caso regular la salida) sin hacer uso de la restricción $\psi(z, \eta, w, u)$, entonces uno podrá estabilizar al sistema (16) y, consecuentemente, al sistema (2). Lo siguiente es consecuencia inmediata de la representación de estados (17). 
Proposición 3.1: Sea $y=z$ la salida del sistema (17). Entonces, el sistema (17) es uniformemente observable. O sea, los estados $(z, \eta)$ pueden ser reconstruidos a partir de las mediciones de $z$.

Es pertinente reparar en que: (a) En el sistema (17), el estado aumentado $\eta$ representa la dinámica de todas las incertidumbres sobre (16), las cuales han sido aglutinadas en $\Theta(z, w, u)$. Pues, $\Theta(z, w, u)$ es interpretada como una variable de estado $\eta$, en un sistema de dimensión 2 (sistema extendido). Dado que $\eta$ es observable (proposición 3.1), su reconstrucción dinámica ofrece un estimado de la función no lineal aglutinante $\Theta(z, w, u) \mathrm{y}$, por consiguiente, de dichas incertidumbres. (b) En una aplicación específica, no se requiere construir la representación de estados (17). Esta sólo será usada como una herramienta para desarrollar al CAR, racionalizar su estructura y analizar la estabilidad a lazo cerrado del sistema resultante.

Recordemos la siguiente noción [19]: Se dice que un sistema es semiglobalmente prácticamente estabilizable en $\mathrm{z}^{*}=0$, si para todos conjuntos compactos $\varnothing_{1} \subset \subset \mathbb{R}$ que contengan a $z^{*}$, existe una ley de control la cual haga del conjunto $\mathbb{E}$ un atractor estable del sistema dentro de $\overbrace{1}$, i.e., alguna trayectoria $z(t)$ del sistema que inicie en ingrese en en un intervalo finito de tiempo. En otras palabras, un sistema es semiglobalmente prácticamente estabilizable en $\mathrm{z}^{*}=0$, si dado un conjunto compacto arbitrario $\$$, todas las trayectorias que inicien dentro de $\%$, éstas pueden ser llevadas a una vecindad de $\mathrm{z}^{*}$, arbitrariamente pequeña, $\mathbb{Q}_{1}$.

Tomemos la salida $y=z$, entonces el sistema (17) tiene grado relativo $\rho=1$. Dado que $\delta^{*}(z, w)=g^{*}(z)+\beta(z, w)$ está acotado sobre cero, por lo tanto, el grado relativo está bien definido para todo $t \geq 0$. Ahora, consideremos la siguiente ley de control

$$
u=\frac{1}{\delta^{*}(z, w)}\left[-\eta-f^{*}(z)-K z\right]
$$

donde $\mathrm{K}>0$. Bajo la ley de control linealizante (18), el estado no medido $\eta$ tiene la siguiente propiedad.

Proposición 3.2: Si la trayectoria $\mathrm{z}(\mathrm{t})$ permanece acotada para todo $\mathrm{t} \geq 0$, entonces el estado aumentado $\eta$ está acotado para todo $t \geq 0$, es decir, bajo el controlador (18) la planta (16) es internamente estable.

Pneeba: Sin pérdida de generalidad y en busca de simplicidad en la presentación de esta prueba, se han omitido los argumentos de las funciones involucradas. Por definición $\eta$ $=f+\delta u$. Tomando la ecuación (18) se tienen que

$$
\eta=\left[\delta^{*} f-\delta\left(f^{*}-\mathrm{Kz}\right)\right] /\left(\delta^{*}+\delta\right)=\left[\delta^{*} f-\delta\left(f^{*}-\mathrm{Kz}\right)\right] /\left(\mathrm{g}^{*}+\mathrm{g}\right)
$$

Dado que todas las funciones involucradas en la expresión anterior son suaves y que $\mathrm{g}^{*}$ es 
Capitulo Tercero

mayor que cero, si $z$ permanece acotada para todo $t \geq 0$, entonces $\eta$ está acotada para todo $t \geq 0$

Ahora, bajo la ley de control (18), se obtiene el siguiente sistema a lazo cerrado

$$
\begin{aligned}
& \dot{z}=-K z \\
& \dot{\eta}=\Xi(z, \eta, W, U) \\
& y=z ; \quad \Psi_{0}=0
\end{aligned}
$$

El sistema anterior tiene estructura de cascada, esto implica que $z \rightarrow 0$ exponencialmente. Dado que la condición $\psi(z, \eta, w, u)=0$ se mantiene para la acción de control (18), $\psi(z, \eta, w, u)=0$ es la primer integral del sistema a lazo cerrado. Así pues, de acuerdo con la proposición 3.2, es evidente que $\eta$ está acotada para todo $t \geq 0$, es decir, las señales del sistema a lazo cerrado son internamente estables.

No obstante, la ley de control linealizante (18) no puede ser implementada directamente por lo siguiente: $f(z, w)$ y $\mathrm{g}(z, w)$ son funciones desconocidas, por lo tanto el estado extendido $\eta=\Theta(z, w, u)=f(z, w)+\delta(z, w) u$ es desconocido. Adicionalmente, no se dispone de él por mediciones.

Empero, es posible explotar la propiedad de observabilidad (proposición 3.1) del sistema (17) para proponer un controlador por retroalimentación de salida. Dado que $f(z, w)$ y $\mathrm{g}(\mathrm{z}, w)$ son funciones inciertas, el término $\Xi(z, \eta, W, U)$ será ignorado en el diseño del estimador de estados. Se propone al siguiente observador de tipo Luenberger

$$
\begin{array}{ll}
\dot{\dot{z}}=\hat{\eta}+f^{*}(z)+\delta^{*}(z, w) u & +\kappa_{1} L(z-\hat{z}) \\
\dot{\hat{\eta}}= & +\kappa_{2} L^{2}(z-\hat{z})
\end{array}
$$

con condiciones iniciales $z(0)=z_{0}$ y $\eta(0)=\eta^{*}$, donde $\eta^{*}$ es un número arbitrario. $z$ y $\eta$ son valores estimados de $z$ y $\eta$, respectivamente. $L \geq L^{*}$ es un parámetro de ajuste y las constantes $\kappa_{1}$ y $\kappa_{2}$ son elegidas de tal manera que el polinomio $P_{2}(s)=s^{2}+\kappa_{1} s+\kappa_{2}=0$ tenga todas sus raíces localizadas en el hemiplano compejo izquierdo, o sea, $P_{2}$ (s) es Hurwitz. Nótese que el estimador (20) es un observador de alta ganancia, su uso está motivado por la idea de recuperar la dinámica de las incertidumbres aglutinadas en $\Theta(z, w, u)$ lo más rápido posible, con el fin de compensarlas. Adviértase que $\eta$ es inicializado arbitrariamente.

Una vez que el estimado $\grave{\eta}$ está disponible, puede ser usado en la ley de control como sigue

$$
u=\frac{1}{g^{*}(z)}\left[-\hat{\eta}-f^{*}(z)-K z\right]
$$

Obviamente, la ley de control (21) sólo depende de funciones conocidas y el valor 
estimado del estado aumentado (o sea, de las incertidumbres aglutinadas). Está claro que el esquema de control propuesto comprende: (a) un compensador dinámico de incertidumbres dado por el estimador (20) y, (b) una ley de control de tipo linealizante, ecuación (21).

Teorema 3.3: Asumamos que las suposiciones (H.3.1)-(H.3.3) se mantienen y seleccionemos el valor de los parámetros $\kappa_{1}$ y $\kappa_{2}$ tales que $P_{2}(s)$ es Hurwitz. Entonces, el CAR (20),(21) es un estabilizador semiglobal práctico para el sistema (16).

Prueba. Sea $e=\left(e_{1}, e_{2}\right) \in \mathbf{R}^{2}$ un vector cuyos componentes están definidos de la siguiente manera: $e_{1}=\mathrm{z}-\hat{z}$ y $e_{2}=\eta-\hat{\eta}$. La dinámica del sistema (17) bajo el CAR (20),(21), es decir a lazo cerrado, está completamente descrita por

$$
\begin{aligned}
& \dot{z}=-K z+\Gamma(e) \\
& \dot{e}=L A(k, r) e+\Phi(z, e, W, u)
\end{aligned}
$$

donde $\Gamma(e)=e_{2}, \Phi(\mathrm{z}, e, W, u)=[0, \phi(\mathrm{z}, e, W, u)]^{\mathrm{T}}, \phi(\mathrm{z}, e, W, u)=\left\{\partial_{\mathrm{x}} f(\mathrm{z}, w)\left[-\mathrm{Kz}+e_{2}\right]+\partial_{\mathrm{w}} f(\mathrm{z}, w) \dot{w}\right.$ $\left.+\partial_{\mathrm{f}} f(\mathrm{z}, w)\right\}+\left\{\partial_{\mathrm{x}} \delta(\mathrm{z}, w)\left[-\mathrm{Kz}+e_{2}\right]+\partial_{\mathrm{w}} \delta(\mathrm{z}, w) w+\partial_{\mathrm{t}} \delta(\mathrm{z}, w)\right\}\left[-\mathrm{Kz}-f(\mathrm{z}, w)+f^{*}(\mathrm{z})+e_{2}\right] /[\delta(\mathrm{z}, w)$ $\left.+\delta^{*}(\mathrm{z}, w)\right]+\delta(\mathrm{z}, w)\left\{\delta_{1} / \delta^{*}(\mathrm{z}, w)+\delta_{2} / \delta(\mathrm{z}, w)\right\}, \delta_{1}=\partial_{\mathrm{x}} f^{*}(\mathrm{z})\left[-\mathrm{Kz}+e_{2}\right]+\partial_{\mathrm{t}} f^{*}(\mathrm{z})$ y $\delta_{2}=$ $\left[\partial_{\mathrm{x}} \delta(\mathrm{z}, w)\left[-\mathrm{Kz}+e_{2}\right]+\partial_{\mathrm{w}} \delta(\mathrm{z}, w) \dot{w}+\partial_{\mathrm{t}} \delta(\mathrm{z}, w)\right] / \delta^{*}(\mathrm{z}, w)$.

A partir de las expresiones anteriores, se obtiene la existencia de tres números positivos independientes de $L$ tales que $\Gamma(0)=0,|\Gamma(e)| \leq|e|$ y $|\phi(z, e, W, u)| \leq \beta_{1}|z|+\beta_{2}|e|+$ $\beta_{3}$. para todo $z \in \mathscr{Q}, e \in \mathbb{R}^{2}, W \in \mathscr{D}$ y $\mathrm{t} \geq 0$. La matriz compañera $\mathrm{A}(\mathrm{\kappa}, \mathrm{r})$ está dada por

$$
A(k, r)=\left[\begin{array}{cc}
-k_{1} & 1 \\
-r k_{2} & 0
\end{array}\right]
$$

donde $r=[\mathrm{g}(\mathrm{z}, w)-\beta(\mathrm{z}, w)] /\left[\mathrm{g}^{*}(\mathrm{z})+\beta(\mathrm{z}, w)\right]$ satisface $0<\varepsilon_{1} \leq r \leq \varepsilon_{2}<1$ para todo $\mathrm{t} \geq 0$. Tomemos el sistema variante en tiempo $e=A(\kappa, r) e$. Luego, del lema en el Apéndice $A$, se tiene que existen dos números positivos $\lambda_{1}$ y $\lambda_{2}$ tales que $|e(t)| \leq \lambda_{1}|e(0)| \exp \left(-\lambda_{2} t\right)$ para todo $\mathrm{t} \geq 0$. Una consecuencia directa de esto es que existe una función cuadrática $\mathrm{V}=e^{\mathrm{T}} \mathrm{P} e$, con $P$ positiva definida, tal que $\nabla \mathrm{VA}(\kappa, r) e \leq-\lambda_{3}|e|$ para un cierto número postivo $\lambda_{3}$.

Con las desigualdades para $|\Gamma(e)|$ y $|\phi(z, e, W, u)|$ y la existencia de la función $\mathrm{V}$, se concluye (a partir del Lema 2.4 en [19], ver Apéndice $B$ ) que existe un número positivo $L^{*}$ $\geq 1$ tal que para todo $\mathrm{L}>\mathrm{L}^{*}$, el compensador dinámico hace a los estados $(\hat{z}, \hat{\eta})$ del controlador (20),(21) uniformemente acotados, y lleva a la trayectoria $z(t)$ del sistema (16) a una vecindad arbitrariamente pequeña $\varnothing_{1}$ del origen siempre que las condiciones iniciales $x_{0}$ estén en $\varangle$. Es decir, para $L>L^{*} \geq 1$, el conjunto $\varangle$ está contenido en la región de atracción del origen.

Obsérvese lo siguiente: Por un lado, en general, $\beta_{1}$ dependerá del tamaño del conjunto 
Capitulo Tercero

Æ, asi que el valor limitante $\mathrm{L}^{*}$ también. Desde el punto de vista de implementación, esto implica que se requiere un valor de la ganancia de estimación $L$ grande, para que un conjunto de condiciones iniciales grande sea estabilizado. Por otro lado, si la desigualdad para $|\phi(z, e, W, u)|$ se mantiene globalmente, entonces el CAR (20),(21) será un estabilizador práctico global. Además, es fácil ver en la expresión de $\phi(z, e, W, u)$ que $|\phi(z, e, W, u)| \leq \beta_{1}|z|+\beta_{2}|e|+$ $\beta_{3}$ se mantiene globalmente si $f(z, w)+f^{*}(z)$ es globalmente Lipschitz y $g(z, w)+g^{*}(z)$ está globalmente acotada.

Para cerrar con el diseño del CAR, es oportuno puntualizar en lo que sigue. (i) Dado que $r=[\mathrm{g}(\mathrm{z}, \mathrm{w})-\beta(\mathrm{z}, \mathrm{w})] /\left[\mathrm{g}^{*}(\mathrm{z})+\beta(\mathrm{z}, w)\right]$ significa una relación entre información desconocida y conocida asociada con la entrada de control. Por consiguiente, si $\mathrm{g}(\mathrm{z}, w)=0$ (no existen incertidumbres asociadas con $u$ ), entonces $\beta(z, w)=0$ y por lo tanto $r=0$. (ii) Dado que $0<$ $g(z, w) \leq \beta(z, w)$, se tiene que $0<\varepsilon_{1} \leq r \leq \varepsilon_{2}<1$ para todo $\mathrm{t} \geq 0$, consecuentemente se satisface esta condición del Teorema 3.3. (iii) Dado que para RCTA $f(z, w), f^{*}(\mathrm{z}), \mathrm{g}(\mathrm{z}, w)$ y $\mathrm{g}^{*}(\mathrm{z})$ son suficientemente suaves con respecto a sus argumentos y globalmente acotadas con respecto a $t, y$ que $w$ y sus derivadas son funciones globalmente acotadas, tenemos que $|\phi(\mathrm{z}, e, W, u)| \leq \beta_{1}+\beta|e|$ para toda $\mathrm{z} \in \varnothing \subset \mathbf{R}$, donde es un conjunto compacto dado que contiene al origen (nótese que $\phi(\cdot)$ no depende de $\mathrm{L}$ ).

Corolario 3.4: El controlador (20),(21) es un estabilizador práctico del RCTA (2).

\subsection{Estabilización asintótica robusta en RCTA's}

Como se mencionó en la sección anterior, el sistema (16) es una forma general de plantas de primer orden. Si además de esto se asume que las propieadas (P.1) y (P.2) se mantienen, entonces el CAR diseñado podrá estabilizar en el origen a un RCTA que pueda escribirse en la forma (17) (Corolario 3.4).

Primero, y con el fin de ligar las ideas desarrolladas en el capítulo 2, consideremos el problema de estabilización de RCTA no isotérmico donde únicamente la tasa de reacción no es conocida. Es decir, se asumirá que $\theta, \gamma$ y $x_{2, \mathrm{e}}$ son conocidas y constantes (H.2.2). Después, se supondrá que únicamente se conocen valores nominales constantes de éstos, pero en realidad tendrán variación en tiempo.

Ejemplo 3.5. Tomemos el RCTA en el ejemplo 2.1 (sistema (2)). El RCTA será estabilizado en $(0.5,400)$ el cual es un punto silla de equilibrio, por medio del controlador (20),(21). Asumamos que no se dispone de información alguna de la cinética de reacción para retroalimentación (el término incierto es la cinética de conversión, nada más). También asúmase que las supocisiones (H.2.1), (H.2.2) y (H.2.3) se cumplen. Esto no modifica los resultados de la sección anterior puesto que (H.2.1), (H.2.2) y (H.2.3) son situaciones particulares de las hipótesis planteadas para desarrollar el CAR (20),(21).

Así pues, $f(x, w)=\beta(x) R(x), f^{*}(x)=\theta\left(x_{2, \mathrm{e}}-x_{2}\right)+\gamma x_{2}, \mathrm{~g}^{*}(x)=\gamma$ y $\mathrm{g}(x, w)=0$ (no existe incertidumbre asociada con la entrada de control) por lo tanto $\beta(z, w)=0$. La función no lineal aglutinante está dada por: $\Theta(z, w, u)=f(z, w)+\delta(z, w) u$, donde $\delta(z, w)=0$, y $\eta=$ 
$\Theta(z, w, u)$. Sea $z=x_{2}-x_{2}^{*}$, donde $x_{2}^{*}$ es la temperatura de referencia. Luego, combinando el sistema (2) con el CAR (20),(21) se obtiene el siguiente sistema a lazo cerrado

$$
\begin{aligned}
& \dot{x}_{1}=\theta\left(x_{1, e}-x_{1}\right)+E R(x, z) \\
& \dot{z}=\theta\left(x_{2, e}-z\right)+\gamma z+\eta+\gamma u \\
& \dot{\hat{z}}=\theta\left(z_{e}-\hat{z}\right)+\gamma \hat{z}+\hat{\eta}+\gamma u+L \kappa_{1}(z-\hat{z}) \\
& \dot{\hat{\eta}}=L^{2} K_{2}(z-\hat{z}) \\
& y=z
\end{aligned}
$$

donde $y$ es la salida, $u=\left[-\theta\left(\mathrm{z}_{\mathrm{e}}-\hat{\mathrm{z}}\right)-\gamma \hat{\mathrm{z}}-\hat{\eta}-\mathrm{Kz}\right] / \gamma, \mathrm{R}(x, \mathrm{z})=x_{1} \mathrm{k}_{0} \exp \left(-\mathrm{Ea} / \mathrm{R} x_{2}\right), \mathrm{E}=1$ es una relación estequiométrica, $\beta(x)=200, \mathrm{Ea} / \mathrm{R}=25, \mathrm{k}_{0}=10^{4}, \theta=\gamma=1, \mathrm{z}_{\mathrm{e}}=1$. El parámetro de control $\mathrm{K}=1.0$, igual que en el ejemplo 2.2. Las contantes $\kappa_{1}$ y $\kappa_{2}$ fueron elegidas de tal forma que $P_{2}(s)$ tenga todas sus raices localizadas en - 1.0. El parámetro de ajuste $\mathrm{L}=15.0$.
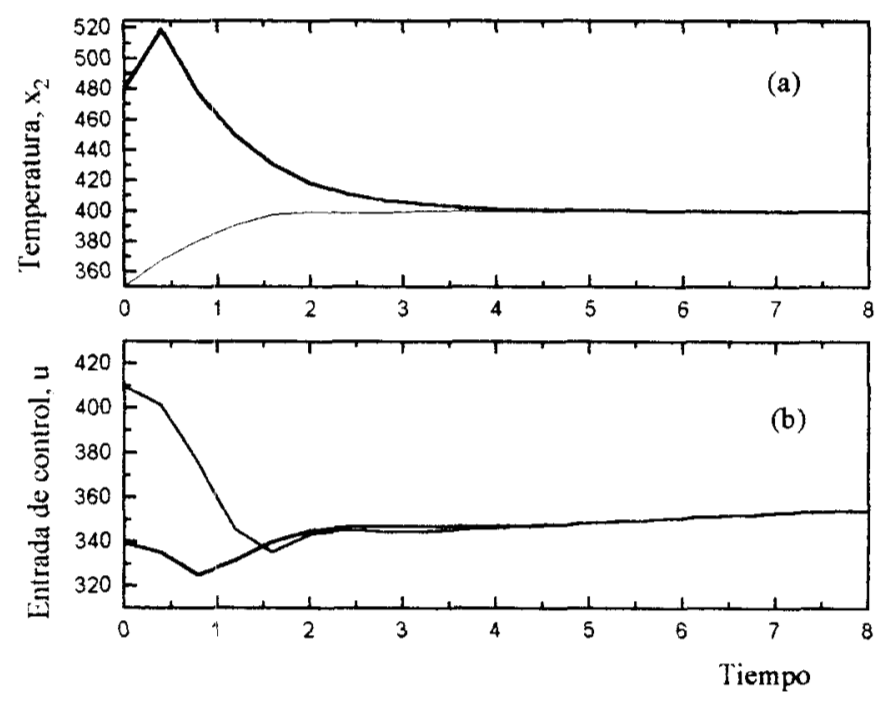

Figura 8. CAR en un RCTA para diferentes condiciones iniciales $(0.3,350)$ línea continua y $(0.8,480)$ discontinua.

En la figura 8 se muestra el desempeño del CAR para dos condiciones iniciales diferentes para $x_{1,0}, z_{0}=\hat{z}_{0}\left(\hat{\eta}_{0}=17\right)$. En la figura 8. a se presenta la regulación de temperatura en $x_{2}^{*}=400$. Debido a que se dispone de un buen estimado del término incierto (mejor que el obtenido con estimación discreta), las acciones de control requeridas para lograr la estabilización son suaves (ver Fig. 8.b). El estimado del valor actual de término incierto se 
Capitulo Tercero

presenta en la figura 9 para las condiciones iniciales $\left(x_{1,9}, \mathbf{z}_{0}, \mathbf{z}_{0}, \eta_{0}\right)=(0.3,350,350,17)$.

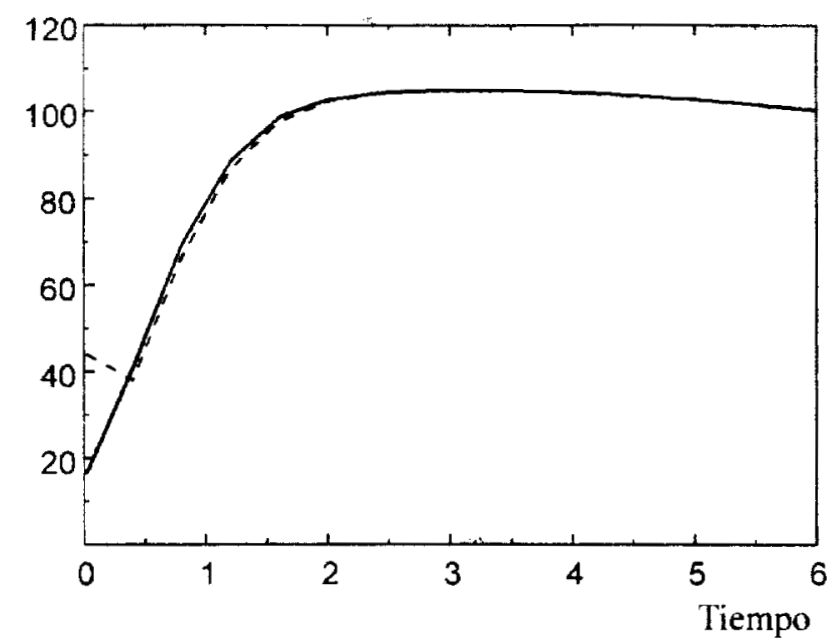

Figura 9. Estimación dinámica de la tasa de conversión, actual; ---- estimada.

En la Figura 10 se presenta el retrato fase del RCTA (24) para diferentes valores del parámetro de ajuste, L. Evidentemente, a mayores valores del alta ganacia de observación, menor es el tamaño de la vecindad donde se estabilizan las trayectorias del sistema (17).

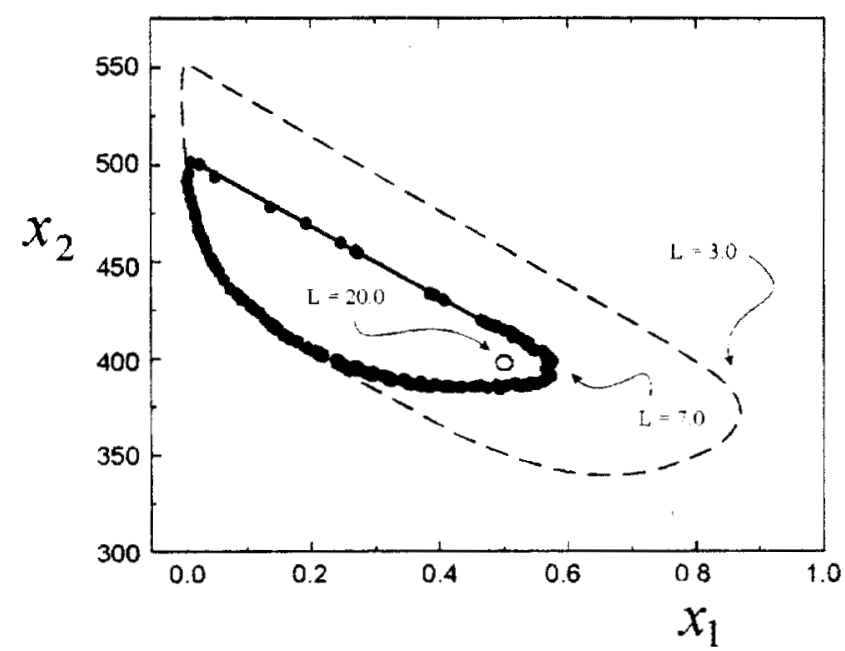

Figura 10. Retrato fase del RCTA (24) a lazo cerrado para diferentes valores de $L$.

Por otra parte, dado que todas las señales del sistema (17) bajo el controlador (20),(21) están acotadas (Proposición 3.2), y que tanto las propiedades del RCTA (P.1) y (P.2) como las hipótesis (H.2.1),(H.2.2.) y (H.2.3) implican que $f(z, w)=\beta(x) R(x)$ es globalmente Lipschitz y $\mathrm{g}(\mathrm{z}, w)+\mathrm{g}^{*}(\mathrm{z})=\gamma$ está globalmente acotada (ver $\left.\$ 2\right)$. Así que, de acuerdo con el 
Teorema 3.3, existe un $\mathrm{L}^{*} \geq 1$ tal que el sistema a lazo cerrado es estable en $z=x_{2}-x_{2}^{*}=0$. En el caso del RCTA del ejemplo $3.4, \mathrm{~L}^{*} \approx 14.7$. Por lo tanto, para cualquier $\mathrm{L}>14.7$ el CAR llevará a las trayectorias $z(t)$ a una vecindad $\bigotimes_{1}$ arbitrariamente pequeña del origen (del orden de $\mathrm{L}^{-1}$ ). En la Figura 11 se muestran los valores propios del Jacobiano del sistema (24). Por supuesto, para valores $L<14.7$ el origen $z=x_{2}-x_{2}^{*}=0$ es localmente inestable.

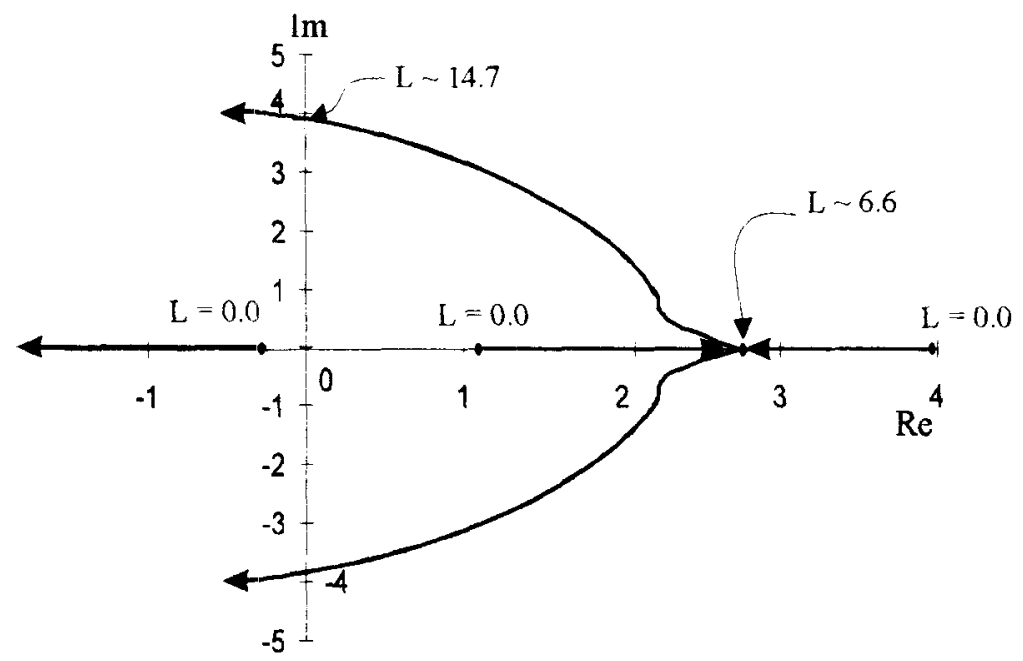

Figura 11. Lugar geométrico de las raíces del Jacobiano del sistema

(24)

Ejemplo 3.6: Tomemos ahora el RCTA del ejemplo anterior, pero bajo las siguientes suposiciones: (i) Solamente la temperatura del reactor está disponible para retroalimentación por mediciones. Un valor nominal de la temperatura de entrada $x_{2, \mathrm{e}}^{*}$ es conocido, pero $x_{2, \mathrm{e}}=$ $x_{2, \mathrm{e}}^{*}+a x(t)$, donde $a$ es un parámetro desconocido y $x(t)$ está acotada. (ii) La cinética de conversión $R(x)$ y la entalpia $\beta(x)$ son desconocidas. (iii) El coeficiente de transferencia de calor $\gamma=\gamma(\mathrm{t})$ es desconocido. Sin embargo, satisface que $0<\gamma_{\min } \leq \gamma \leq \gamma_{\max } \leq \infty$. Todas estas suposiciones son realistas y no contravienen las hipótesis (H.3.1), (H.3.2) y (H.3.3)

Si adicionalmente consideramos que el vector de concentraciones $x_{1, \mathrm{e}} \leq x_{1, \mathrm{e}}^{*} \in \mathbf{R}^{\mathrm{m}}$, entonces por conservación de masa se tiene que $x_{1 . e}$ está acotada para todo $t \geq 0$ [2]. Definiendo $\mathrm{z}=x_{2}-x_{2}^{*} \mathrm{y}$ tomando al vector $w=x_{1}$, la dinámica del RCTA puede ser descrita por la ecuación (16), donde $f(x, w)=\theta a x_{1, \mathrm{e}}+\beta(x) R\left(w, x_{2}\right)+\gamma x_{2}, f^{*}(z)=\theta\left(x_{1, \mathrm{e}}^{*}-\mathrm{z}\right)-\gamma_{\max } z$, $\mathrm{g}(x, w)=\gamma-\gamma_{\max } \mathrm{y} \mathrm{g}^{*}(\mathrm{z})=\gamma_{\max }$. Nótese que $\mathrm{g}(x, w) \leq 0$ por lo tanto se puede tomar el valor de la cota $\beta(x, w)=0$. Así, la función no lineal aglutinante está dada por: $\Theta(z, w, u)=f(z, w)+$ $\delta(z, w) u$ y $\eta=\Theta(z, w, u)$, donde $\delta(z, w)=\gamma-\gamma_{\max }$.

Luego, el sistema a lazo cerrado es 
Capítulo Tercero

$$
\begin{aligned}
& \dot{z}=\theta\left(z_{e}-z\right)+\beta(z) R(w, z)-\gamma(z-u) \\
& \dot{\hat{z}}=\hat{\eta}+\theta\left(z_{e}^{*}-z\right)+\gamma_{\max } u+L \kappa_{1}(z-\hat{z}) \\
& \dot{\hat{\eta}}=L^{2} \kappa_{2}(z-\hat{z}) \\
& y=z
\end{aligned}
$$

donde $u=\left[-\theta\left(z_{\mathrm{e}}^{*}-\mathrm{z}\right)-\hat{\eta}+\mathrm{Kz}\right] / \gamma_{\max }, \mathrm{K}<0$. La estabilidad práctica global (para $x_{2}=\mathrm{z}+$ $x_{2}^{*}$, donde $x_{2}^{*}$ es una referencia constante), es obtenida si $\beta(z) R(w, z)$ es globalmente Lipschitz con respecto a $x_{2}$. Esto no es dificil de probar si la cinética de reacción está dada por expresiones de tipo Arrenhius [2].

Para mostrar el desempeño del CAR, supóngase que $w=x_{1} \in \mathbf{R}$ y que la reacción que se lleva a cabo en el RCTA es $\mathrm{A} \rightarrow \mathrm{B}$ cuya cinética está dada por $R\left(w, x_{2}\right)=w \mathrm{k}_{0} \exp \left(-\mathrm{Ea} / \mathrm{R} x_{2}\right)$. Así pues, la dinámica de la señal de pertubación estará dada por el subsistema (2.1), por lo tanto $w=\theta\left(w_{\mathrm{c}}-w\right)+\mathrm{E} R\left(w, x_{2}\right)$, donde $\mathrm{E}=1$ es la relación estequiométrica entre producto y reactante, $w_{\mathrm{e}}=1$ y $\theta=1$ es el inverso del tiempo de residencia del reactante en el RCTA.

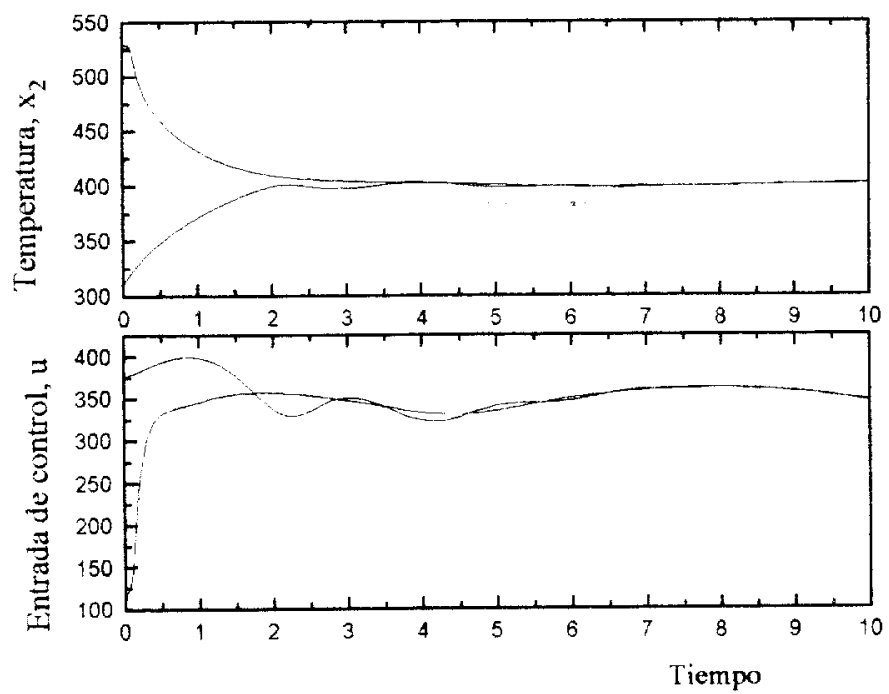

Figura 12. Estabilización de un RCTA a pesar de errores en modelo y perturbaciones.

Además, tómese los siguientes valores: $z_{\mathrm{e}}=z_{\mathrm{e}}^{*}+a x_{2, \mathrm{e}}=350+5.0 \operatorname{sen}(\mathrm{t}), \gamma=1+$ $0.2 \cos (\mathrm{z}), \mathrm{k}_{0}=\exp (25), \mathrm{Ea} / \mathrm{R}=200, \gamma_{\max }=2$ y $x_{2}^{*}=400$. El parámetro de control $\mathrm{K}=1.0$, las constantes $\kappa_{1}$ y $\kappa_{2}$ fueron elegidas tales que $P_{2}(s)$ tenga sus raíces en -1.0 , finalmente, $L$ $=17$. En la Figura 12 se muestra la estabilización del RCTA para diferentes condiciones iniciales. Nótese que la temperatura $x_{2}$ converge suavemente a la referencia deseada $x_{2}^{*}$, es 
decir, $\mathrm{z} \rightarrow 0$ cuando $\mathrm{t} \rightarrow x$, a pesar de las incertidumbres en el coeficiente de transferencia de calor, errores en modelo y perturbaciones en la planta. Las oscilaciones transcientes en la variable de control son debidas a la dependencia directa del coeficiente de transferencia de calor $\gamma$ con la temperatura.

Dada la analogía entre los esquemas discreto y continuo (y las propiedades de las tasa específica de crecimiento de biomasa, ver $\mathrm{Ej} .2 .2$ ), los reactores biológicos pueden ser controlados mediante el CAR (20),(21). Además, cabe señalar que el CAR (20),(21) es capaz de regular la composición de salida en una columna de destilación rectificadora [7].

\subsection{Una interpretación clásica del CAR}

Para fines de control, los procesos químicos pueden ser modelados obteniendo su operador de transferencia entrada-salida, $G(s)=Y(s) / U(s)$. Más aún, muchos procesos resultan en una planta de primer orden. Las plantas de primer orden son capaces de representar la dinámica en el rango de frecuencia de interés para el diseño de un control por retroalimentación en procesos químicos [20]. Ejemplos de esto son: columnas de destilación rectificadoras [7],[21], intercambiadores de calor [8] y RCTA [20].

En general, a partir de la identificación del sistema mediante el análisis de respuesta de su salida ante cambios en escalón de la entrada, algunos procesos (incluyendo los antes mencionados) son modelados por

$$
G(s)=\frac{K_{p} e^{-T s}}{\tau s+1}
$$

donde $\mathrm{T}$ es el tiempo de retardo, $\mathrm{K}_{\mathrm{p}}$ es la ganancia estática de la planta y $\tau$ es su tiempo característico. El modelo (26) es muy conveniente puesto que únicamente contiene tres parámetros. Pueden usarse pruebas de retardo para estimar los valores nominales de $T, K_{p} y$ $\tau$ [17]. En particular, y con el fin de que los resultados duscutidos en esta sección sean comparativos a aquellos de las secciones 3.1 y 3.2 , asumiremos que $\mathrm{T}=0$.

Es posible controlar la planta (26) usando retroalimentación proporcional integral (PI). Aproximaciones de control basadas en modelo interno (IMC por sus siglas en inglés) permiten sintonizarle adecuadamente [22]. En casos prácticos, una sintonización de controladores PIIMC ofrece un aceptable margen de robustez frente a pequeñas incertidumbres y perturbaciones de carga estática. Sin que esto signifique que un control PI tenga propiedades de robustez ante variaciones dinámicas en las pertubaciones ni ante errores de modelado [23]. Dado el fenómeno de degradación del desempeño (wind up) del controladores PI, varios autores han estudiado técnicas para evitar los efectos indeseables que dicha degradación del control pueda provocar (ver [5], [23], [24] y referencias ahi citadas). En esta sección se interpreta el CAR diseñado anteriormente, como un control PI robusto ante cargas dinámicas (incluyendo variaciones paramétricas en la planta (26)) sin este fenómeno de degradación. 
Capitulo Tercero

Primero recordemos brevemente los fundamentos de control clásico. Para ello consideremos un canal de perturbaciones acotadas y desconocidas (ver Figura 1) $d=d(\mathrm{t}$ ) de entrada a la planta y asumamos que $T=0$. Entonces, del modelo (26) se puede escribir a la salida del sistema como sigue

$$
Y(s)=\frac{K_{p}}{\tau s+1}[U(s)+D(s)]
$$

en dominio de tiempo, la planta (27) queda $\dot{y}=-y / \tau+\mathrm{K}_{1} u+\mathrm{K}_{\mathrm{p}} d / \tau$. También en dominio de tiempo, un controlador PI puede ser escrito como: $u=\left(1 / \tau-1 / \tau_{\mathrm{c}}\right) / \mathrm{K}_{1}-\mathrm{z}, \mathrm{z}=\mathrm{K}_{2} \mathrm{z}$, donde $\tau_{\mathrm{c}}$ es el tiempo característico del control y $z=y-y^{*}$ es el error de desviación. Bajo el control PI anterior, $\mathrm{K}_{1}$ y $\mathrm{K}_{2}$ son las ganancias proporcional e integral, respectivamente. Luego, el sistema a lazo cerrado estará dado por el siguiente sistema de ecuaciones diferenciales

$$
\begin{aligned}
& \dot{y}=-y / \tau-K_{1} z+K_{p} d / \tau \\
& \dot{z}=K_{2} z
\end{aligned}
$$

Si el controlador PI lleva al sistema asintóticamente a un punto equilibrio, $y_{\mathrm{eq}}=0 \mathrm{y}$ $\mathrm{z}_{\mathrm{eq}}=\left(\mathrm{K}_{\mathrm{p}} / \tau\right) d_{\infty}$. Cuando $\mathrm{t} \rightarrow \infty$, la variable integral estima asintóticamente al valor de la carga estática $d_{x}=\lim _{\mathrm{t} \rightarrow \infty} d(\mathrm{t})$. De tal manera, $d_{x}$ es contrarrestada por $\mathrm{z}_{\mathrm{eq}}$ y se suprime el sesgo en estado estacionario. Más aún, por argumentos de continuidad, es de esperarse que $z=z(t)$ sea un estimado de $d$ a bajas frecuencias. De hecho, el valor de la ganancia integral $\mathrm{K}_{2}$ puede ser interpretada como una frecuencia de corte para perturbaciones cuya dinámica puede ser estimada por el error de desviación [20].

Ahora bien, siguiendo las ideas desarrolladas en las secciones anteriores, incluyendo las del $\$ 2$. Notemos que a partir de la representación de la planta (27) en dominio de tiempo (es decir, $\dot{y}=-y / \tau+\mathrm{K}_{1} u+\mathrm{K}_{\mathrm{p}} d / \tau$ ), y si la perturbación $d$ es continua en $\mathrm{t}$, se puede escribir como el sistema de estados en espacio extendido dado por

$$
\begin{aligned}
& \dot{y}=-y / \tau+K_{1} u+K_{p} d / \tau \\
& \dot{d}=F(t)
\end{aligned}
$$

donde $F(\mathrm{t})$ es una función continua, acotada y desconocida. Por comparación entre el sistema (29) con el sistema (17), es evidente que se puede reconstruir a la dinámica de las perturbaciones $d$ mediante el observador (20), y por lo tanto el uso de la ley de control (21) estabilizará asintóticamente a la planta (26), pero con estimación dinámica de perturbaciones de carga de altas frecuencias (para una comparación congruente, cabe señalar que $(z, \hat{z}, \hat{\eta})=$ $(y, \hat{y}, \eta)$, donde $\eta=d)$.

Dado que la planta (26), el estimador de estados (20) y el controlador (21) son lineales con respecto a los estados y la perturbación $\eta=d$, luego de algunas sencillas manipulaciones algebraicas, se obtiene la siguiente función de transferencia del control 


$$
C(s)=K_{c}\left[1+\frac{1}{\tau_{l} s}+\frac{K_{e}}{s\left(s+L K_{1}\right)}\right]
$$

donde $K_{c}=\Upsilon / K_{1}, \tau_{I}^{-1}=L^{2} K_{2} \Upsilon, K_{e}=L^{2} K_{2}\left(L K_{1}-1 / \tau_{c}\right) \Upsilon$ y $\Upsilon=\left(1 / \tau-1 / \tau_{c}\right)$. Así pues, el operador de transferencia del control está compuesto por tres términos: (i) Una retroalimentación proporcional $\mathrm{K}_{\mathrm{c}}$, (ii) Una acción integral, $\mathrm{K}_{\mathrm{c}} / \tau_{\mathrm{I}} \mathrm{s} \mathrm{y}$, (iii) Una acción integral de segundo orden, $\mathrm{K}_{\mathrm{c}} \mathrm{K}_{\mathrm{e}} /\left[\mathrm{s}\left(\mathrm{s}+\mathrm{L} \kappa_{1}\right)\right]$. La ley de control (30) ha sido llamada $\mathrm{PII}^{2}$ debido a la presencia de la acción integral al cuadrado.

La acción integral de segundo orden acrecenta la capacidad de estimación del controlador PI clásico, permitiendo la estimación dinámica de la perturbación $d$. Debe advertirse que eligiendo $K_{e}=0$, (o equivalentemente, $L=1 / \kappa_{1} \tau_{c}$ ), la retroalimentación (30) se reduce a un PI clásico [5]. El siguiente ejemplo ilustra la estimación dinámica de una perturbación oscilante. Se puede observar que a mayor frecuencia de la señal perturbante se requiere un mayor valor del parámetro de estimación $L$ (por ello, $L$ ha sido llamada ganancia de alta frecuencia).

Ejemplo 3.7: Consideremos un RCTA cuyo operador de transferencia está dado por la planta (27) (ver [20]). Asúmase que $\mathrm{K}_{\mathrm{p}}=1$ y $\tau=1$ y supóngase que el RCTA está sometido a una perturbación oscilante de amplitud unitaria, $d=\operatorname{sen}(2 \pi \omega \mathrm{t})$.

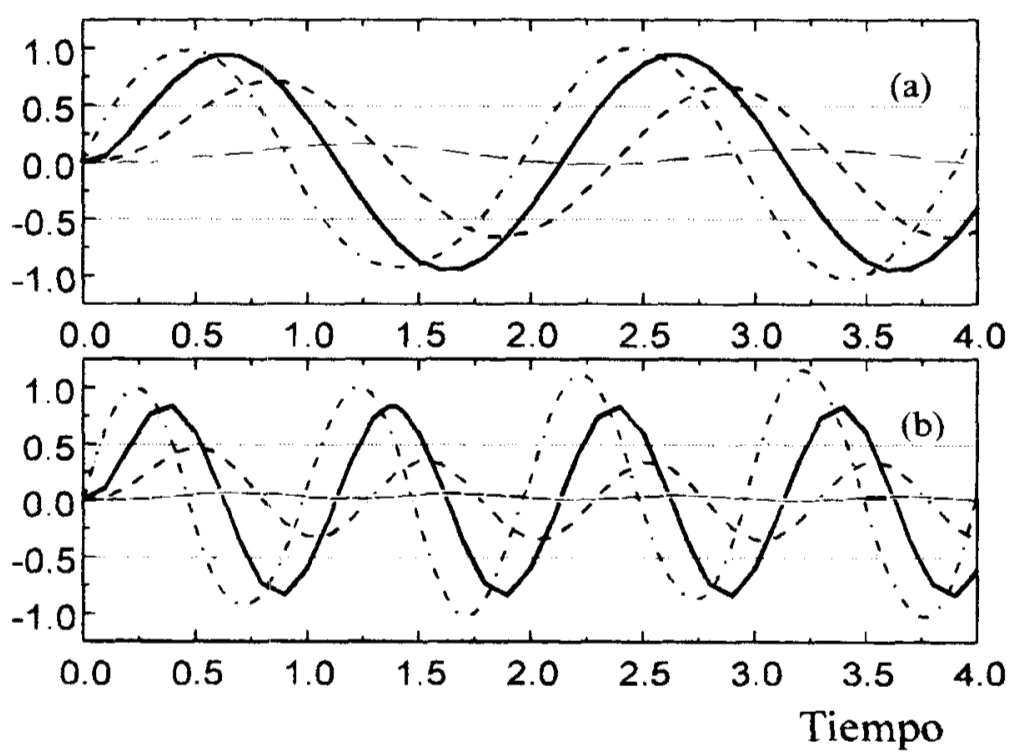

Figura 13. Estimación de perturbación. Guión (punto) $d(\mathrm{t})$, (fino) $L=1$, (grueso) $L=5.0$ y continua $L=15$.

Tomemos al tiempo característico $\tau_{c}=\frac{2}{3} \tau$ (lo cual implica que el sistema a lazo cerrado es ligeramente más rápido que a lazo abierto; esta regla heurística es común en procesos 
quimicos [20]). En la Figura 13 se muestra la estimación dinámica de la perturbación simulada para tres valores diferentes de L (los valores son: 1.0, 5.0 y 15.0). En la figura 13.a se presenta el caso donde $\omega=0.5$. Si L crece la estimación de perturbaciones es mejor, lo cual se corrobora para el caso donde $\omega=1.0$. (ver Figura 13.b). De hecho en el primer caso para $L=15.0$, la estimación es aceptable, y para el segundo existe mayor error. Obsérvese que para el segundo caso (Fig. 13.b) cuando $L=5.0$ la señal estimada tiene amplitud menor de 0.5 , mientras que para $\omega=0.5$ y $L=5.0$ la amplitud de la señal de $\hat{\eta}$ es mayor que 0.5 (Fig. 13.a). Asi pues, si L crece entonces $\eta \rightarrow \eta=d$, entonces el CAR (30) (o su equivalente, ecuación (21)) podrá contrarrestar el efecto de la perturbación en la entrada de RCTA.

Por otra parte, la mayoría de las veces nada más se dispone de valores nominales de los parámetros de la planta (27). No obstante, aún en estos casos es posible utilizar una ley de control del tipo $\mathrm{PII}^{2}$. Sean $\mathrm{K}_{\mathrm{p}}^{*}$ y $\tau^{*}$ los valores nominales de la planta (es decir unos estimados de $\mathrm{K}_{\mathrm{p}} \mathrm{y} \tau$, respectivamente). Luego, la ecuación (27) puede ser reescrita en dominio de tiempo como sigue: $\dot{y}=-y / \tau+\mathrm{K}_{\mathrm{p}}^{*} u+w$, donde $w=d-\left(1 / \tau-1 / \tau^{*}\right) y+\left(\mathrm{K}_{\mathrm{p}}-\mathrm{K}_{\mathrm{p}}^{*}\right) u$. Nótese que $w$ ha aglutinado a las incertidumbres asociadas a la perturbación dinámica de carga y las variaciones paramétricas.

Si vemos a $w$ como una perturbación, la dinámica de la planta anterior puede ser descrita por la representación de estados en espacio extendido (29), donde en lugar de la ecuación (29.2) (la cual describe la dinámica de $w$ ) se tiene que:

$$
\dot{w}=\Xi(y, u, \dot{u}, d, d)=\dot{d}-\left(1 / \tau-1 / \tau^{*}\right)\left(-y / \tau+\mathrm{K}_{\mathrm{p}}^{*} u\right)+\left(\mathrm{K}_{\mathrm{p}}-\mathrm{K}_{\mathrm{p}}^{*}\right) \dot{u}
$$

Claro está que $\Xi(y, u, \dot{u}, d, \dot{d})$ es una función desconocida y acotada. Por consiguiente, al igual que en casos anteriores se puede utilizar el algoritmo de diseño y obtener un CAR. Para probar estabilidad se puede seguir el procedimiento reportado en [5].

Recordemos que, de acuerdo con el resultado en el Apéndice $A$, la relación de ganancia entrada-salida, $r$, impone restricciones sobre la estabilidad del sistema a lazo cerrado. Una condición necesaria para que el sistema a lazo cerrado sea internamente estable es que $r>0$. En el caso de la planta $(27), r=\mathrm{K}_{\mathrm{p}} / \mathrm{K}_{\mathrm{p}}^{*}$, lo cual implica que para garantizar estabilidad interna $\operatorname{sgn}\left(\mathrm{K}_{\mathrm{p}}\right)=\operatorname{sgn}\left(\mathrm{K}_{\mathrm{p}}^{*}\right)$. Esto último se satisface por la suposición (H.3.1). Evidentemente, esto no es una restricción muy fuerte ya que en la enorme mayoría de los procesos químicos el signo de la ganacia estática $\mathrm{K}_{\mathrm{p}}$ es conocido [20].

Ejemplo 3.8: Consideremos el RCTA del ejemplo 3.7. Pero ahora pensemos que existe incertidumbre paramétrica. Asumamos que $\tau$ es exactamente conocido, $K_{\mathrm{p}} \in\left[\mathrm{K}_{\mathrm{p}}^{\mathrm{sup}}, \mathrm{K}_{\mathrm{p}}^{\text {ini }}\right]=$ $[0.25,2]$ y que una carga estática $d=-1.0$ actúa sobre el proceso a $t=10 . y^{*}=0.0, L=1.15$ y las constantes $\kappa_{1}$ y $\kappa_{2}$ son tales que la matriz $A(r, \kappa)$ (ecuación (23)) tiene todos sus valores propios localizados en -1.0 . En la Figura 14 se muestra el desempeño del control para $K_{p}^{*}=$ $\mathrm{K}_{\mathrm{p}}^{\text {sup }}, \mathrm{K}_{\mathrm{p}}^{*}=\mathrm{K}_{\mathrm{p}}^{\mathrm{inf}}$ y $\mathrm{K}_{\mathrm{p}}^{*}=\left(\mathrm{K}_{\mathrm{p}}^{\text {sup }}+\mathrm{K}_{\mathrm{p}}^{\text {inf }}\right) / 2$. El mejor desempeño se alcanza cuando $\mathrm{K}_{\mathrm{p}}^{*}=\left(\mathrm{K}_{\mathrm{p}}^{\text {sup }}+\right.$ $\mathrm{K}_{\mathrm{p}}^{\mathrm{inf}}$ )/2, donde se exhibe rapidez de convergencia aceptable cojugada a un esfuerzo de control moderado. 
Aquí es pertinente decir lo siguiente: (i) $\mathrm{Si} T=0$, el estimador dinámico de perturbaciones (20) toma la forma del modelo de la planta, el CAR resultante induce estabilidad al sistema a lazo cerrado. (ii) Sin embargo, cuando $T \neq 0$, el estimador dinámico es no causal. O sea, se requiere estimar las incertidumbres en el 'futuro' (en un tiempo $t+T$ ). Por supuesto, que con el algoritmo propuesto este requerimiento de estimación no es posible. $Y$ aunque alguna aproximación ha sido propuesta en [5], son necesarias modificaciones que permitan predecir dichas incertidumbres. Cabe señalar que los sistemas con retardos son por demás interesantes, sin embargo así mismo presentan dificultad. Es de esperar que la presencia de retardo induzca restricciones al desempeño y eficiencia de cualquier controlador, no existe razón alguna para pensar que CAR se excluya de esto.
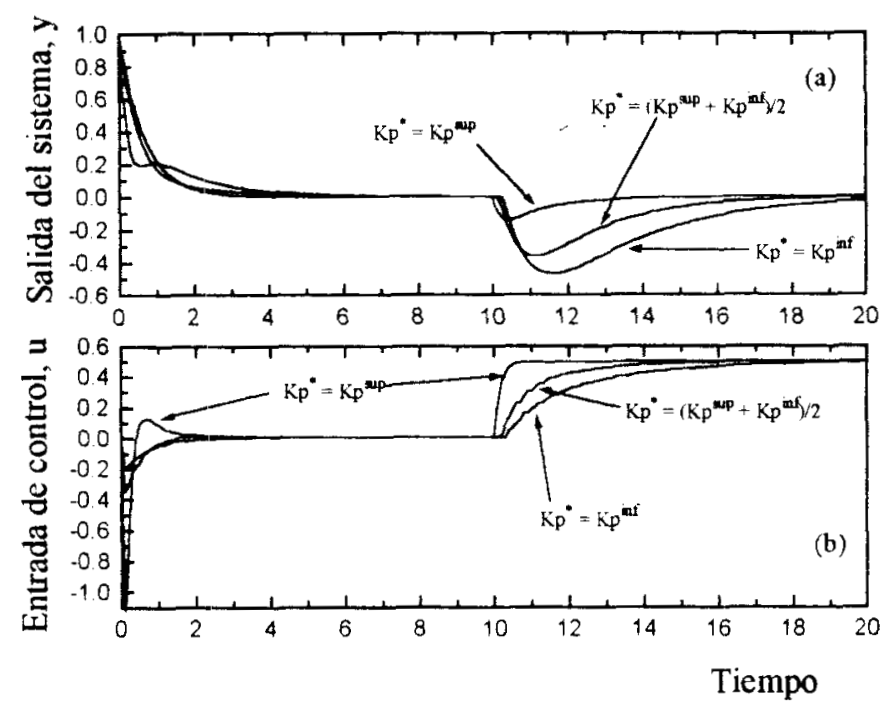

Figura 14. Desempeño del $\mathrm{PII}^{2}$ a diferentes $\mathrm{K}_{\mathrm{p}}^{*}$.

\subsection{Un ejemplo para comparar desempeño}

Evidentemente, el CAR resultante tiene una estructura de control similar a la de esquemas adaptativos. Pues, se ha elegido una clase de reactor con el fin de comparar el desempeño del CAR con el de algoritmos adaptativos. Además, se ha incluido una comparación del CAR con un controlador PI-IMC. Para tal fin, se toma una clase de RCTA para el cual ya hayan sido desarrollados y reportados algoritmos adaptativos.

Consideremos un biorreactor de alimentación continua descrito por el siguiente modelo en espacio de estados [18], [25]

$$
\begin{gathered}
\dot{x}_{1}=[\mu(x)-u] x_{1} \\
\dot{x}_{2}=-Y_{\mathrm{s}} \mu(x) x_{1}+\left[\mathrm{s}_{\mathrm{e}}-x_{2}\right] u \\
y=x_{2}
\end{gathered}
$$


donde $x_{1}, x_{2} \in \mathbb{R}$ son la concentración de biomasa y sustrato, respectivamente. $y \in \mathbb{R}$ es la salida del biorreactor. $Y_{s}$ denota al coeficiente de crecimiento de biomasa, $s_{c}=s_{e}(t)$ es una función acotada que representa la concentración de sustrato alimentada al biorreactor, $u$ es la tasa de dilución (variable manipulada). Por ultimo, $\mu(x)$ es la tasa especifica de crecimiento de biomasa. Como ya se ha dicho, $\mu(x)$ es una función muy compleja de varios factores físicos y biológicos, por ello es difícil tener una expresión exacta para este término. Asúmase que, a partir del diseño del equipo, se conocen valores nominales de: (i) La concetración de sustrato a la entrada y, (ii) la tasa de dilución.

Obtengamos el modelo nominal considerando el estado estacionario del sistema que gobierna la dinámica del biorreactor (i.e., $\dot{x}_{1}=0$ y $\dot{x}_{2}=0$ ). Luego, se tienen las siguientes condiciones de equilibrio [26]: (i) $x_{1}^{*}=\left(\mathrm{s}_{\mathrm{e}}^{*}-x_{1}^{*}\right) / \mathrm{Y}_{\mathrm{s}} \mathrm{y} x_{2}^{*}=\mathrm{K}_{\mathrm{M}} u^{*} /\left(\mu_{\max }-u^{*}\right)$, donde $\mathrm{s}_{\mathrm{e}}^{*} \mathrm{y} u^{*}$ son valores nominales de $\mathrm{s}_{\mathrm{e}}$ y $u$, respectivamente. (ii) $0<x_{2}^{*}<\mathrm{s}_{\mathrm{e}}^{*}$ y $0<x_{2}^{*}<\mathrm{Y}_{\mathrm{s}} \mathrm{s}_{\mathrm{e}}^{*}$. Cabe decir que la primer condición corresponde al comportamiento deseado mientras que el equilibrio dado por la segunda es la indeseable condición de lavado del biorreactor.

$$
\begin{aligned}
\text { Definiendo } z=x_{2}-x_{2}^{*}, w & =x_{1}-x_{1}^{*} \text { se puede reescribir el modelo de biorreactor como } \\
\dot{z} & =-Y_{s} \mu(z, w)+\left[s_{e}-z\right] u
\end{aligned}
$$

donde $w$ representa a la perturbación que actua sobre el reactor cuya dinámica está dada por $\dot{w}=[\mu(z, w)-u] w$ (dinámica de la concentración de bimasa). Una ley de control linealizante para el sistema (31) está dada por

$$
u=\frac{Y_{s} \mu(z, w)+V(z)}{s_{e}-z}
$$

donde $V(z)=-K_{1} z$. La ley de control linealizante (32) no puede ser físicamente realizada porque requiere el conocimiento perfecto de la expresion de tasa de crecimiento de biomasa y mediciones de las perturbaciones a la entrada (representadas en la concentración de sustrato). Se han propuesto diversas estrategias para aproximar al término incierto no lineal. Se han propuesto tanto algoritmos adaptativos lineales (ver [16], [17] y referencias ahí citadas), como no lineales [26]. Más aún, en [25] y [27] los autores hicieron una comparación de desempeño entre diferentes esquemas de control de tipo adaptativo. A continuación se describen brevemente los algoritmos a comparar.

\subsubsection{El controlador PI-IMC.}

En la sección 3.3 se discutieron las similitudes y diferencias que guardan el CAR y un control clásico PI. Para la identificación del operador de transferencia entrada-salida se indujo en la entrada (tasa de dilución) un salto en escalón de $5 \%$ para obtener la respuesta de la salida (concentración de sustrato) que resulta en una planta de primer orden sin retardo (ecuación (27)). Los parámetros de la planta son: $\mathrm{K}_{\mathrm{p}}=32, \tau=12, \mathrm{~T}=0$. Considerando un canal de perturbaciones exógenas, la planta puede escribirse, en dominio de tiempo, la ley de control está dado por: $u(\mathrm{~s})=\mathrm{K}_{1}+\mathrm{K}_{2} / \tau_{\mathrm{c}} \mathrm{s}$, donde $\mathrm{K}_{1}$ es la ganancia proporcional y $\mathrm{K}_{2}$ es la 
ganancia integral del control. El controlador PI fue sintonizado mediante la metodología basada en control de moderno interno [22]. El procedimiento es el siguiente: Tomando la derivada en tiempo del sistema a lazo cerrado, se obtiene una ecuación diferencial de segundo orden cuyos coeficientes están asociados con $\mathrm{K}_{1} \mathrm{y} \mathrm{K}_{2}$. Luego, por asignación de polos, uno tiene que $K_{1}=\lambda^{*} \tau / K_{p}$ y $K_{2}=\lambda^{*} / K_{p}$ donde $\lambda^{*}<0$.

\subsubsection{Control adaptativo lineal (CAL)}

Las estrategias adaptativas han sido diseñadas para tomar en cuenta las características no estacionarias de biorreactores [26],[27]. Tales esquemas de control parten de un modelo en espacio de estados. A fin de implementar un controlador adaptativo se diseña un esquema de adaptación para ajustar los parémetros del modelo de referencia y obtener, por este medio, valores estimados de las incertidumbres. Esencialmente, se propone una función con parámetros desconocidos variantes en tiempo. En particular el $\mathrm{CAL}$ requiere una parametrización lineal del modelo de referencia. De hecho, esto es una desventaja de los CAL's, puesto que la tasa específica de crecimiento es no lineal en parámetros, un biorreactor, cualquiera, también lo es (ver discusión de la sección 2.3). Así pues, definiendo $\alpha_{\mathrm{L}}(x ; \pi)=$ $\mu(x)$, donde $\pi=\left(\pi_{1}, \pi_{2}\right)$ es un conjunto de parámetros desconocidos variantes en tiempo. El modelo de referencia se puede parametrizar de la siguiente forma: $\alpha_{\mathrm{L}}(x, \pi)=\pi_{1} x_{1}+\pi_{2} x_{2}$. De esta manera, la ley adaptable de control (32) es: $u_{\mathrm{CAL}}=\left(\pi_{1} x_{1}+\pi_{2} x_{2}+K_{1} z\right) /\left(s_{e}^{*}-z\right)$. Nótese que para implementar fisicamente la ley adaptable de control anterior, se requeren mediciones de concetración de biomasa, $x_{1}$. Esto es otra desventaja del esquema adaptativo lineal reportado [25], [27]. Adicionalmente, dado que $\pi_{1}$ y $\pi_{2}$ son desconocidos, se deben ajustar mediante un algoritmo adaptativo. En [26] se propuso el siguiente esquema de adaptación paramétrica: $\mathrm{d} \pi_{\mathrm{j}}^{\mathrm{e}} / \mathrm{dt}=\mathrm{g}_{\mathrm{j}} \pi_{\mathrm{j}}^{\mathrm{e}}$, donde $\mathrm{g}_{\mathrm{j}}$ son las ganancias de adaptación, $\mathrm{j}=1,2$. Es decir se añaden tantas ecuaciones diferenciales como parámetros se tengan (una desventaja más). Luego el controlador adaptable es: $u_{\mathrm{CAL}}=\left(\alpha_{\mathrm{L}}\left(x ; \pi^{\mathrm{e}}\right)+\mathrm{K}_{1} z\right) /\left(\mathrm{s}_{\mathrm{e}}^{*}-z\right)$. Obsérvese que en el controlador adaptable lineal se deben sintonizar $\mathrm{j}+\mathrm{l}$ parámetros de control (las ganacias de adaptación y la constante de control $\mathrm{K}_{\mathrm{l}}$ ).

\subsubsection{Control adaptativo no lineal (CANL)}

Al contrario de un esquema adaptativo lineal, en un controlador adaptable no lineal, el modelo de referencia se parametriza no-linealmente [26], [27]. Definieno $\alpha_{\mathrm{NL}}(x ; \pi)=\mu(x)$, donde $\pi=\left(\pi_{1}, \pi_{2}\right)$ es un conjunto de parámetros desconocidos variantes en tiempo, se puede parametrizar al modelo de referencia como sigue: $\alpha_{\mathrm{NL}}(x ; \pi)=-\pi_{1} x_{1} /\left(1+\pi_{2} x_{2}\right)$. Cabe señalar que la parametrización propuesta en [26] tiene la misma forma que la ley de Monod. Además, se asume que los parámetros $\pi_{1}$ y $\pi_{2}$ pertenecen a un conjunto compacto conocido, $\pi \in \mathscr{F}_{\pi} \subset$ $\mathbf{R}^{2}$, tal que

$$
\mathscr{F}_{\pi}=\left\{\pi: \pi=\left[\pi_{1}, \pi_{2}\right]=\left[\mu_{\mathrm{M}}, \mathrm{K}_{\mathrm{M}}\right], \mu_{\mathrm{M}}=\left[\mu_{\mathrm{m}, n}, \mu_{\mathrm{max}}\right], \mathrm{K}_{\mathrm{M}}=\left[\mathrm{K}_{\mathrm{min}}, \mathrm{K}_{\max }\right]\right\}
$$

Es decir, el esquema adaptativo no lineal requiere información acerca de la parametrización real de las incertidumbres y cotas sobre los parámetros. Luego, la ley 
adaptable de control es: $\boldsymbol{u}_{C+\mathrm{NI}}=\left(\alpha_{\mathrm{NL}}(x ; \pi)+\mathrm{K}, z\right) /\left(\mathrm{s}_{\mathrm{e}}^{*}-\mathrm{z}\right)$. Los parámetros deben ser ajustados mediante leyes de adaptación. En [26], [27] los autores propusieron lo siguiente

$$
\dot{\pi}_{j}^{e}=\operatorname{Proj}_{\pi_{j}}\left\{\frac{-g_{j} x_{1} x_{2} z}{1+\pi_{j} x_{2}}\left(1-\frac{\operatorname{sgn}\left(x_{1} z\right)\left(\pi_{j, \max }-2 \pi_{j, \min }^{e}+\pi_{j}^{e}\right)}{G_{j}\left(\pi_{j, \max }+2 \varepsilon_{j}-\pi_{j, \min }\right)\left(\pi_{j, \max }-\pi_{j}^{e}-\varepsilon_{j}\right)+1}\right)\right\}
$$

$\varepsilon_{\mathrm{j}}=\min \left\{\pi_{\mathrm{j}, \mathrm{min}},\left(\pi_{\mathrm{j}, \max }-\pi_{\mathrm{j}, \mathrm{min}}\right) / 2\right\}, \pi_{\mathrm{j}} \in\left[\left(\pi_{\mathrm{j} \text { min }}-\varepsilon_{\mathrm{j}}\right),\left(\pi_{\mathrm{j}, \max }+\varepsilon_{\mathrm{j}}\right)\right], \mathrm{G}_{\mathrm{j}}$ y $\mathrm{g}_{\mathrm{j}}$ son ganancias de ajuste, $\mathrm{j}=1,2$. La función $\operatorname{Proj}\{\cdot\}$ denota al operador de proyección cuyo papel es asegurar que los

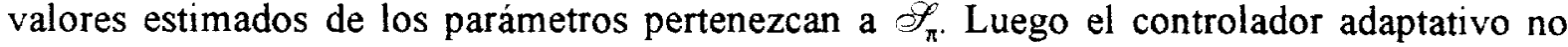
lineal es: $u_{\mathrm{CANL}}=\left(\alpha_{\mathrm{NL}}\left(x ; \pi^{\mathrm{e}}\right)+\mathrm{K}_{l} z\right) /\left(s_{\mathrm{e}}^{*}-z\right)$, al igual que el caso lineal, la anterior ley de control requiere: (a) La integración de un número de ecuaciones diferenciales igual al número de parámetros del modelo de referencia, (b) información acerca de la estructura paramética de las incertidumbres y (c) mediciones de concentración de biomasa. Empero, por añadidura, es necesario sintonizar $2 j+1$ parámetros de control (acoplados). En [25] y [27] se ha reconocido la dificultad que presentan los algoritmos adaptativos para su sintonzado.

\subsubsection{El controlador asintótico robusto (CAR)}

Definiendo $\delta=\mathrm{s}_{\mathrm{e}}-\mathrm{s}_{\mathrm{e}}^{*}, \delta^{*}=\mathrm{s}_{\mathrm{e}}^{*}, f(\mathrm{z}, w)=-\mathrm{Y}_{\mathrm{s}} \mu(\mathrm{z}, w), f^{*}(\mathrm{z}, w)=0, \Theta(\mathrm{z}, w, u)=f(\mathrm{z}, w)+$ $\delta u$, y $\eta=\Theta(z, w, u)$, el sistema de primer orden (31) puede ser reescrito como el siguiente sistema extendido

$$
\begin{gathered}
\dot{\mathrm{z}}=\eta+\left(\delta^{*}-\mathrm{z}\right) \boldsymbol{u} \\
\dot{\eta}=\Xi(\mathrm{z}, \eta, W, U) \\
y=z
\end{gathered}
$$

donde $\Xi(z, \eta, W, U)$ está dada como en la ecuación (17). A fin de reconstruir la dinámica del estado no medido, $\eta$, el observador (20) toma la forma

$$
\begin{aligned}
& \dot{\hat{z}}=\eta+\left(\delta^{*}-z\right) u+L \kappa_{1}(z-\hat{z}) \\
& \dot{\eta}=\quad+L^{\prime} K_{2}(z-\hat{z})
\end{aligned}
$$

donde $\kappa_{1}$ y $\kappa_{2}$ son elegidas en tal forma que $s^{2}+\kappa_{1} s+\kappa_{2}=0$ es Hurwitz y $L>L^{*}>0$. Luego el controlador está dado por: $u=\left[-\hat{\eta}+K_{1} z\right] / \delta^{*}(z)$. Nótese que para implementar el CAR sólo es necesario integrar 2 ecuaciones diferenciales (independientemente del número de parámetros involucrados en la tasa específica de crecimiento). Se necesita la menor información disponible respecto al modelo $(\mu(x)$ es función suave y Lipschitz), se tienen dos parámetros de control, $\mathrm{L}$ y $\mathrm{K}_{1}$, los cuales están desacoplados. Además, sólo se requiere que: (a) La entrada $s_{\mathfrak{e}}=s_{\mathrm{e}}(t)$ sea acotada $y$, (b) $s_{\mathrm{c}}^{*} \neq \mathrm{z}$ (el grado relativo esté bien definido para todo $z$ ). 


\subsubsection{Criterio de desempeño}

Para discutir sobre el desempeño alcanzado por cada uno de los controladores mencionados anteriormente, se implementaron por medio de simulaciones numéricas. Las simulaciones fueron hechas de acuerdo con lo siguiente: (i) $\mathrm{At}=15 \mathrm{Hr}$, se indujo un cambio de referencia de $2.0 \mathrm{~g} / \mathrm{L}$ a $2.5 \mathrm{~g} / \mathrm{L}(+25 \%) \mathrm{y}$, (ii) se impuso el siguiente comportamiento oscilatorio a la concentración de sustrato de entrada, $s_{e}=s_{e}^{*}+\operatorname{sen}(\omega t),\left(\omega=0.15 \mathrm{Hr}^{-1}\right)$. Adicionalmente, $\mathrm{s}_{\mathrm{e}}^{*}=5.0$ y se supuso que la tasa específica de crecimiento estaba dada por la ley de Monod (es decir, $\mu(x)=\mu_{\max } x_{2} /\left(\mathrm{K}_{\mathrm{M}}+x_{2}\right)$, donde $\mu_{\max }=5.0, \mathrm{~K}_{\mathrm{M}}=2.0$ y $\mathrm{Y}_{\mathrm{s}}=0.33$ ). Los parámetros de control fueron elegidos como (en particular para los esquemas adaptativos se obtuvo una sintonización aceptable luego de exhaustivas sesiones de simulación): (i) PIIMC, $\mathrm{K}_{1}=-0.93, \mathrm{~K}_{2}=-0.078$, (ii) $\mathrm{CAL}, \mathrm{K}_{1}=-1.0, \mathrm{~g}_{1}=\mathrm{g}_{2}=0.1$, (iii) CANL, $\mathrm{K}_{1}=1.02$, $\mathrm{g}_{1}=1.0, \mathrm{~g}_{2}=10.0, \mathrm{G}_{1}=10.0, \mathrm{G}_{2}=100.0 \mathrm{y}$, (iv) CAR, $\mathrm{L}=0.25, \mathrm{~K}_{1}=1.0$ (las constantes $\kappa_{1}$ y $\kappa_{2}$ son tales que $s^{2}+\kappa_{1} s+\kappa_{2}=0$ tiene todas sus raices localizadas en -1 ).

A fin de obtener una comparación cuantitativa, se consideró el siguiente criterio

$$
J=\int_{0}^{t^{*}}\left\{e_{s n}^{2}+e_{d n}^{2}\right\} d t
$$

donde $e_{\mathrm{sn}}=\left(x_{2}-x_{2}^{*}\right) / x_{2}^{*}$ y $e=\left(u-u^{*}\right) / u^{*}$. $e_{\mathrm{sn}}$ significa la distancia entre el valor de concentración de sustrato y la referencia en cada tiempo mientras que $e_{\mathrm{dn}}$ denota el esfuerzo de control realizado para llevar a $x_{2}$ a la referencia. Los resultados se presentan en la Tabla I, evidentemente combinando el criterio se tiene una cuantifización de que tan rápido se regula el sustrato con menor esfuerzo de control. Es obvia la ventaja que presenta para este caso el CAR.

Tabla I. Valores promedio del indicador de desempeño por controlador

\begin{tabular}{|c|c|c|c|}
\hline Controlador & $e_{\mathrm{sn}}^{2}$ & $e_{\mathrm{dn}}^{2}$ & $J$ \\
\hline PI-IMC & 5.631 & 0.2769 & 5.908 \\
\hline CAL & 3.828 & 0.2707 & 4.099 \\
\hline CANL & 6.092 & 0.2535 & 6.346 \\
\hline CAR & 3.450 & 0.2649 & 3.175 \\
\hline
\end{tabular}

\subsection{Observaciones y comentarios sobre el CAR para sistemas de primer orden}

Al momento se ha desarrollado un algoritmo de control asintótico robusto ante errores en modelo, variaciones paramétricas y señales perturbantes para sistemas de primer orden. Mediante la aglutinación de incertidumbres en una función no lineal, queda clara la interpretación de dicha función aglutinante como una variable de estado en un sistema en 
espacio extendido (dimensión 2). Luego, se propone un estimador de estados para el sistema en espacio extendido. Así, se provee a una ley de control linealizante con un estimado dinámico de las incertidumbres con el objetivo de contrarrestar su efecto en la planta. Entonces, el CAR comprende dos partes: (a) Un estimador dinámico de incertidumbres y (b) una ley de control de tipo linealizante.

Se propuso como estimador dinámico de incertidumbres a un observador de estados de tipo Luenberger. Ello facilita el análisis de estabilidad a lazo cerrado y permite que se logre una indubitable interpretación clásica. Para interpretar clásicamente al CAR, se obtuvo el operador de transferencia del control y se compararon los términos involucrados con los del bien conocido controlador PI. El CAR introduce un término integral de segundo orden el cual permite obtener el estimado dinámico de las señales perturbantes. Así que, usando el CAR es posible eliminar el efecto que presentan perturbaciones de carga no sólo a bajas frecuencias. De este modo, la interpretación clásica del CAR redunda en que el parámetro de alta ganancia, L, es una ganancia de alta frecuencia.

Por otro lado, en este capítulo se plantearon hipótesis que relajan en parte a aquellas del $\S 2$. Aunque, para garantizar estabilidad global se requiere que la función que describe la información asociada a la entrada de control sea globalmente Lipschitz. En los sistemas reactantes dicha función es, al menos, semiglobalmente Lipschitz. Esto es suficiente para probar estabilidad semiglobal. Garantizar la estabilidad semiglobal de un proceso quimicos (incluyendo los RCTA's) es suficiente para fines prácticos. No obstante, desde el punto de vista de control de sistemas, es conveniente relajar tal condición. Esto se hará en el siguiente capítulo.

Al momento, se ha considerado un modelo de un RCTA que ignora la dinámica de temperatura del medio enfriante/calentante. Lo cual resulta en una planta de primer orden. En este caso, la temperatura de la chaqueta en la variable manipulada. Aunque los RCTA's son buenos candidatos para establecer diseños de control en cascada, el impacto de la inclusión de la dinámica de temperatura de chaqueta en el comportamiento dinámico de algún reactor puede ser catastrófico [8],[9]. Recientemente se ha probado que la inserción de la dinámica de chaqueta en el modelo tiene un efecto importante sobre el comportamiento de multiplicidad de estados estacionarios. Por ejemplo, en [8] se demostró que la inclusión de la dinámica de chaqueta en el modelo puede inducir inestabilidad en el reactor aún cuando el lazo-interno se estabilizado por retroalimentación. Adicionalmente, la sensibilidad paramétrica del modelo de tres estados puede exhibir trayectorias caóticas [9].

Por ello, es conveniente extender el esquema de linealización asintótica robusta hacia sistemas de orden superior. Donde el comportamiento dinámico de los RCTA's esté representado por plantas de orden mayor a uno. Esto será presentado en el siguiente capítulo, en el cual de abordan reactores de lecho fluidizado con reacción heterogénea, reactores biológicos de lote alimentado y otro tipo de proceso (como el enfriamiento de producto en efluente de proceso). 
Control Robusto de Reactores Quimicos: Un esquema de control para plantas de primer orden

Capitulo cuarto

\section{Linealización Asintótica Robusta de Procesos Químicos}


Capitulo cuarto

Los resultados presentados en este capitulo fueron extraidos y complementados de aquellos publicados en los artículos, según la relación presentada en el prefacio. Deseo hacer del conocimiento del lector mi agradecimiento a todos los coautores de dichos trabajos. 


\section{Linealización Asintótical Robusta de Procesos Químicos}

En este capítulo se presenta el diseño de CAR para sistemas de orden superior. Buscando claridad en la presentación, la primer sección contiene un breve repaso de los conceptos acerca de linealización de sistemas de orden superior mediante la retroalimentación de mediciones (tomando como plataforma a la teoría de control basada en geometría diferencial). Luego, y siguiendo las ideas descritas previamente, se expone el algoritmo de diseño para lograr controlar asintóticamente al sistema. El controlador resultante es robusto ante errores en modelo, variaciones paramétricas y perturbaciones exógenas. Se consideran algunos procesos para ilustrar el desempeño y discutir características relevantes del CAR desarrollado.

\subsection{Linealización de sistemas no lineales de orden superior}

Con el fin de diseñar al CAR, se hacen dos suposiciones intermedarias para obtener el algoritmo final de linealización asintótica robusta. Así en forma 'pasajera' se asumirá que: (a) Los campos vectoriales del sistema son exactamente conocidos (no hay errores en modelo) y, (b) que todos lo estados están disponibles para retroalimentación. Por supuesto que estas suposiciones no son realistas, pero sólo son utilizadas como hipótesis intermedias, así que ambas serán relajadas.

\subsubsection{Linealización por retroalimentación de estados}

Considérese el siguiente sistema no lineal de orden superior

$$
\begin{aligned}
& \dot{x}=f(x, w)+g(x, w) u \\
& y=h(x)
\end{aligned}
$$

donde $x \in \mathbb{R}^{\mathrm{n}}$ es un vector de estados, $f(x, w)$ y $\mathrm{g}(x, w)$ son campos vectoriales suaves, $h(x)$ es una función suave, $u \in \mathbf{R}$ es la entrada de control, $w$ es un vector de señales de perturbación y $y \in \mathbb{R}$ es la salida del sistema. Por simplicidad en la presentación, de aquí en adelante se omitirá en la notación la dependencia del sistema con $w$.

Diferenciando la salida $y$ respecto al tiempo se obtiene $y=\mathscr{L}_{f} h(x)+\mathscr{L}_{g} h(x) u$, donde $\mathscr{L} h(x)=\sum_{i-1}^{\mathrm{n}} f_{\mathrm{i}}(x ; \pi) \partial h(x) / \partial x$ es la derivada de Lie de $h(x)$ respecto a $f(x ; \pi)$. Si $\mathscr{L} h(x)=0$ para todo $x$, uno debe derivar de nuevo, con lo cual se tiene que: $\ddot{y}=\mathscr{L}_{f}^{2} h(x)+\mathscr{L}_{g} \mathscr{L}_{f} h(x) u$. Más generalmente, si $\rho$ es el número entero más pequeño tal que:

$$
\text { (i) } \mathscr{L}_{g} \mathscr{L}_{f}^{\mathrm{i}} h(x)=0, \mathrm{i}=1,2, \ldots, \rho-2
$$

(ii) $\mathscr{L}_{g} \mathscr{L}_{f}^{p-1} h(x) \neq 0$

entonces, uno puede escribir las primeras $\rho$ derivadas de la salida $y$ como sigue 
Capitulo cuarto

$$
\begin{gathered}
y^{(1)}=\mathscr{L}_{f} h(x) \\
y^{(p)}=\mathscr{L}_{f} h(x)+\mathscr{L}_{3} \mathscr{L}_{f}^{p-1} h(x) u
\end{gathered}
$$

donde $\mathscr{L}_{f}^{1} h(x)=\mathscr{L}_{f}\left(\mathscr{L}_{f}^{1-1} h(x)\right)$ y $\mathscr{L}_{g} \mathscr{L}_{f}^{1} h(x)=\mathscr{L}_{g}\left(\mathscr{L}_{f}^{1} h(x)\right)$.

Si las condiciones (i) y (ii) se mantienen para todo $x=x_{0}$, se dice que el sistema (31) tiene grado relativo $\rho$ en $x_{0}$ [1]. Luego, definiendo $\rho$ nuevas coordenadas $z_{1-1}=y^{(i)}=\mathscr{L}_{f}^{i} h(x)$, $1 \leq \mathrm{i} \leq \rho$, el sistema (31) puede ser reescrito en la siguiente forma canónica

$$
\begin{aligned}
\dot{z}_{i} & =z_{i+1}, \quad i=1,2, \ldots, \rho-1 \\
\dot{z}_{p} & =\alpha(z, v)+\gamma(z, v) u \\
\dot{v} & =\zeta(z, v) \\
y & =z_{1}
\end{aligned}
$$

donde $z \in \mathbb{R}^{\rho}$ son estados observables, $v \in \mathbb{R}^{n-p}$ son estados no obervables, $\alpha(z, v)=\mathscr{L}_{j}^{p} h(x)$ y $\gamma(z, v)=\mathscr{L}_{z} \mathscr{L}_{f}^{p-1} h(x) . \dot{v}=\zeta(z, v)$ se le conoce como dinámica interna del sistema (35). Particularmente, si el sistema (35) se estabiliza en el origen, al subsistema $v=\zeta(z, v)$ se le llama dinámica cero. Si $\gamma(z, v)$ es acotada y es estrictamente diferente de cero para todo $x$ se dice que el grado relativo $\rho$ está bien definido. En tal caso, es fácil ver que la ley de retroalimentación dada por $u=\left[-\mathscr{L}_{f}^{\infty} h(x)+\mathrm{V}(x)\right] / \mathscr{L}_{\mathrm{g}} \mathscr{L}_{f}^{\infty-1} h(x)$, donde $\mathrm{V}(x)$ es una función lineal de los estados $z \in \mathbb{R}^{p}$, es una ley de control linealizante. Además, si

$$
\mathrm{V}(x)=y_{\mathrm{r}}^{(p)}+\mathrm{K}_{p}\left[y_{\mathrm{r}}^{(\rho-1)}-\mathscr{L}_{f}^{p-1} h(x)\right]+\ldots+\mathrm{K}_{\mathrm{i}}\left[y_{\mathrm{r}}-y\right]
$$

donde las $\mathrm{K}_{1}^{\prime} \mathrm{s}(\mathrm{i}=1,2, \ldots, \rho)$ son tales que el polinomio

$$
\mathbf{P}_{\rho}(\mathbf{s})=\mathbf{s}^{\rho}+\mathrm{K}_{\rho} \mathbf{s}^{\rho-1}+\ldots+\mathrm{K}_{2} \mathbf{s}+\mathrm{K}_{1}=0
$$

es Hurwitz y $y_{\mathrm{r}}, y_{\mathrm{r}}{ }^{1}, \ldots, y_{\mathrm{r}}{ }^{\rho}$ son la señal de referencia y sus derivadas, la ley de control linealizante produce el seguimiento asintótico de las trayectoiras de (34) sobre $y_{\mathrm{r}}[1]$.

Asumamos que el sistema (35) es de fase mínima. Sin pérdida de generalidad consideremos que $y_{\mathrm{r}}=y^{*}$ y $z_{1}=y-y^{*}$. En tal caso, la anterior ley de control linealizante para el sistema (35) puede ser escrita en coordenadas $(z, v)$ como

$$
u=\frac{1}{\gamma(z, v)}\left[-\alpha(z, v)+K^{T} z\right]
$$

y llevará a las trayectorias de (35) asintóticamente establemente a cero. Más aún, la dinámica de (35) a lazo cerrado viene a ser 


$$
\begin{gathered}
\dot{z}=A z \\
\dot{v}=\zeta(z, v)
\end{gathered}
$$

donde $\mathrm{A} \in \mathbf{R}^{p \cdot p}$ es la matriz compañera cuya ecuación característica está dada por el polinomio $P_{p}(s)$ (que es Hurwitz). Dado que por suposición el sistema (35) es de fase mínima y la matriz A es Hurwitz, entonces el sistema a lazo cerrado es estable en el origen. No obstante, la ley de control (36) fue obtenida asumiendo que los estados $z \in \mathbb{R}^{\rho}$ están disponibles para retroalimentación. Ahora, empecemos por relajar las suposiciónes intermediarias.

\subsubsection{Linealización por retroalimentación de mediciones}

La aplicación de la linealización entrada-salida basada en geometría diferencial en procesos químicos es muy promisoria porque no se requiere derivar un "linealizador" por retroalimentación de estados. Así pues, si no es viable obtener mediciones de todos los estados $\mathbf{z} \in \mathbf{R}^{\rho}$, la ley de control linealizante (36) no puede implementarse directamente. De hecho, se requiere de un estimado de ellos. Existen algunos trabajos en los que han reportado resultados al problema de estabilización mediante retroalimentación de salida (por ejemplo ver $[15],[28],[29],[30],[31],[32])$.

Luego, se puede obtener el siguiente estimador de estados

$$
\begin{aligned}
& \dot{z}_{i}=\hat{z}_{i+1}+L^{i} x_{i}\left(z_{1}-\hat{z}_{1}\right), \quad 1 \leq i \leq \rho \\
& \dot{z}_{\rho}=\alpha(\hat{z}, v)+\gamma(\hat{z}, v)+L^{\rho} \kappa_{\rho}\left(z_{1}-\hat{z}_{1}\right)
\end{aligned}
$$

donde las constante positivas $\kappa_{i}^{\prime} s, i=1,2, \ldots, \rho$, son elegidas tales que el polinomio $P_{\rho}(s)$ es Hurwitz. L es un parámetro de ajuste (parámetro de estimación de alta frecuencia). Así la ley de control linealizante (36) es reescrita como

$$
u=\frac{1}{\gamma(\hat{z}, v)}\left[-\alpha(\hat{z}, v)+K^{T} \hat{z}\right]
$$

donde $\mathrm{K}_{\mathrm{L}}^{\prime} \mathrm{s}$ son elegidas tales que $\mathrm{P}_{\mathrm{p}}(\mathrm{s})$ es Hurwitz.

Ahora bien, acciones de control retroalimentado basadas en observadores de alta ganancia pueden inducir efectos dinámicos indeseables como el fenómeno de 'pico' [33], [34]. Este fenómeno se caracteriza por inestabilidades a lazo cerrado representadas por escapes a tiempo finito y grandes acciones de control (sobre disparos). Para disminuir el efecto de estas inestabilidades, puede modificarse al controlador (38) por medio de la siguiente función de saturación

$$
u^{s}=\operatorname{Sat}\left\{\frac{1}{\gamma(\hat{z}, v)}\left[-\alpha(\hat{z}, v)+K^{T} \hat{z}\right]\right\}
$$

donde $\operatorname{Sat}\{\cdot\}: \mathbf{R} \rightarrow \not 3$ es una función de saturación y $\not 3 \subset \mathbf{R}$ es un conjunto acotado. Más aún, 
Capitulo cuarto

es posible probar que el controlador por retroalimentación de salida (39) es un estabilizador asintótico semiglobal para el sistema (34) (para un bosquejo de la prueba ver Apéndice B o los resultados en [28]).

Un observador de estados resuelve el problema de la falta de medición de $z$. Sin embargo, si los campos vectoriales $f(x, w)$ y $\mathrm{g}(x, w)$ son desconocidos, el cambio de coordenadas $z=T(x)$ es incierto (aunque invertible). Por consiguiente las funciones $\alpha(z, v)$ y $\gamma(z, v)$ son desconocidas y consecuentemente $\alpha(\hat{z}, v)$ y $\gamma(z, v)$ también lo son. Adicionalmente, la construcción de un estimador de estados no elimina el problema asociado con la no medición de los estados no observables $v \in \mathbf{R}^{n-p}$. Por lo tanto, las funciones $\alpha(z, v)$ y $\gamma(z, v)$ no pueden ser utilizadas en la ley de control linealizante (39) ni en la construcción del observador (37). No obstante, siguiendo las ideas de las secciones en los capítulos pasados y con el antecedente que es posible reconstruir la dinámica de los estados del sistema en forma canónica (35), continuemos por terminar de relajar las suposiciones intermediarias.

\subsection{Estabilización por retroalimentación de mediciones bajo campos vectoriales inciertos}

En los subsecuentes párrafos, se elaborará un algoritmo de diseño que considere incertidumbres asociadas a errores en modelo, variaciones paramétricas y perturbaciones. Para este fin asumamos lo siguiente:

H.4.1) La salida del sistema (35) es $y=z_{1}$ y está disponible para retroaliemtación.

H.4.2) $\gamma(z, v)$ está acotada y es estrictamente diferente de cero.

H.4.3) Las funciones no lineales $\alpha(z, v)$ y $\gamma(z . v)$ son desconocidas. Empero, se dispone de un estimado $\hat{\gamma}(z)$ (función suave) para retroalimentación, tal que $\operatorname{sgn}(\hat{\gamma}(z))=\operatorname{sgn}(\gamma(z, v))$.

H.4.4) El sistema (35) es de fase mínima.

De las anteriores, (H.4.1) es clara y resulta redundante mencionar algo al respecto. En lo que concierne a (H.4.2) y (H.4.3), nótese que ahora sólo se pide que la función asociada con la información de entrada sea diferente de cero (es decir, que el grado relativo esté bien definido para todo $x$ ) y que se conozca su signo. La hipótesis (H.4.4) es la más fuerte. Afortunadamente, la enorme mayoría de procesos químicos la cumplen.

Consideremos al sistema en forma canónica (35). Definamos lo siguiente: Sea $\delta(z, v)$ $=\gamma(z, v)-\gamma(z), \Theta(z, v, u)=\alpha(z, v)+\delta(z, v) u$ y $\eta=\Theta(z, v, u)$. Luego, el sistema (35) puede ser reescrito como

$$
\begin{aligned}
\dot{z}_{i} & =z_{i}+1, \quad 1 \leq i \leq \rho-1 \\
\dot{z}_{\rho} & =\eta+\dot{\gamma}(z) u \\
\dot{\eta} & =\Xi(z, v, \eta, u) \\
\dot{v} & =\zeta(z, v) \\
y & =z_{1}
\end{aligned}
$$


donde $\Xi(z, v, \eta, u)=\sum_{k-1}^{p-1} z_{k-1} \partial_{k} \Theta(z, v, u)+[\eta+\gamma(z) u] \partial_{p} \Theta(z, v, u)+\delta(z, v) u+\partial_{v} \Theta(z, v, u) \zeta(z, v)$, $\hat{c}_{\mathrm{k}} \Theta(z, v, u)=\hat{c} \Theta(z, v, u) / \hat{c} z_{k}$.

Una vez más resulta directo probar que $\psi(z, \eta, u)=\Theta(z, v, u)-\eta=0$ es una primer integral (restricción algebraica) y $\psi_{0}=\psi\left(z_{0}, \eta_{0}, u_{0}\right)$, donde el subindice 0 significa condición inicial. Lo cual implica que el sistema $(40)$ es dinámicamente externamente equivalente al sistema (35). Esto significa que los sistemas (35) y (40) tienen las mismas soluciones siempre que se satisfaga la restricción $\psi_{0}=0$. De hecho, la superficie $\psi(z, \eta, u)=0$ es invariante bajo la dinámica del sistema $(40)$, es decir, $d \psi(z, \eta, u) / d t=0$ sobre las trayectorias generadas por el sistema (40) [18].

En la Figura 15 se ilustra la noción geométrica del estado extendido $\eta$. Al respecto cabe puntualizar que: (a) Si $z(t)$ es una solución de (35) con condiciones iniciales $\left(z_{0}, v_{0} ; \boldsymbol{u}_{0}\right)$ y $(z(t), \eta(t))$ es una solución para $(40)$ con condiciones iniciales $\left(z_{0}, \eta_{0}, v_{0} ; \boldsymbol{u}_{0}\right)$, entonces la igualdad $\psi(z, \eta, u)=0$ y la condición $\mathrm{d} \psi(z, \eta, u) / \mathrm{dt}=0$ implican que la solución de (35) es una proyección de (40). (b) Luego, en el sistema (40), el estado aumentado $\eta$ representa todas las incertidumbres que actúan sobre (35), las cuales han sido aglutinadas en $\Theta(z, v, u)$. Dado que $\eta$ es observable (proposición 3.1), su reconstrucción dinámica ofrece un estimado de la función no lineal aglutinante $\Theta(z, v, u) y$, por consiguiente, de dichas incertidumbres. Por lo tanto, si uno es capaz de estabilizar en el origen al sistema (40) (o en su caso regular la salida) $\sin$ hacer uso de la restricción algebraica $\psi(z, \eta, u)$, entonces uno podrá estabilizar al sistema (35) (y por consecuencia al sistema (34)). (c) En una aplicación específica, no se requiere construir la representación de estados (40). Esta sólo será usada como una

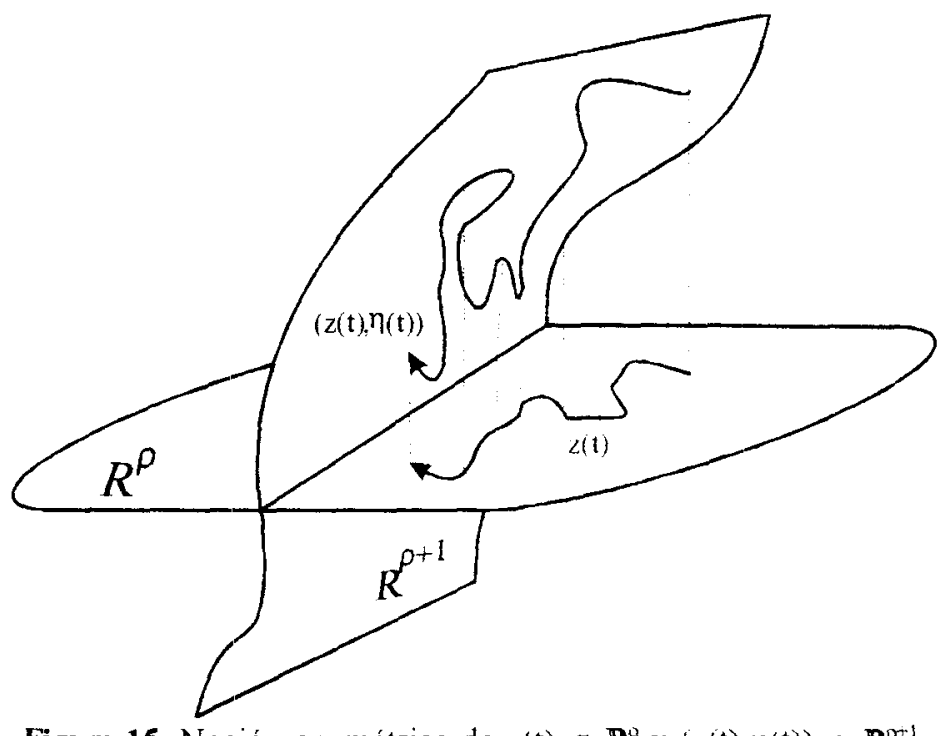

Figura 15. Noción geométrica de $z(t) \in \mathbb{R}^{p} y(z(t), \eta(t)) \in \mathbb{R}^{p+1}$.

herramienta para desarrollar al CAR, racionalizar su estructura y analizar la estabilidad a lazo cerrado del sistema resultante. (d) El sistema (40) es dinámicamente externamente equivalente al sistema (35). 
Capitulo cuario

Proposición 4.1: Consideremos la ley de control dada por

$$
u=\frac{1}{\hat{\gamma}(z)}\left[-\eta+K^{T} z\right]
$$

donde las constantes $\mathrm{K}^{\prime}$ 's son tales que el polinomio $\mathrm{P}_{\mathrm{p}}(\mathrm{s})$ es Hurwitz. Si las condiciones iniciales satisfacen $\psi_{0}=\psi\left(z_{0}, \eta_{0}, u_{0}\right)=0$, entonces, bajo el control $u$ los estados de (37) convergen a cero.

Prueba: La convergencia a cero se sigue del hecho que el sistema a lazo cerrado está en la forma de cascada. Y dado que $f(x ; \pi)$ es suave, también $\alpha(z, v)$ es suave. Por consiguiente la dinámica del control está dada por

$$
\dot{u}=\frac{-\Xi(z, \eta v, u)+K^{T_{\dot{z}}}}{\hat{\gamma}(z)}+\frac{\left[-\eta+K^{T} z\right\} \dot{z} \partial_{z} \hat{\gamma}(z)}{\hat{\gamma}(z)^{2}}
$$

donde $\partial_{z} \gamma(z)=\partial \hat{\gamma}(z) / \partial z$. Dado que $\Xi(z, \eta, v, u)$ es una función suave, $\dot{u}$ también es suave. Por lo tanto, $\psi(z, \eta, u)=\Theta(z, u)-\eta$ es la primer integral del sistema (40). Consecuentemente, de acuerdo con la proposición 3.2, $\eta$ está acotada. Por último, dado que $\mathrm{z}$ converge exponencialmente a cero, entonces $\eta$ converge exponencialmente a cero.

Por otro lado, solamente $y=z_{1}$ está disponible para retroalimentación por mediciones. Entonces la ley de control en la proposición 4.1 no puede ser implementada directamente. Sin embargo, tal y como fue establecido en la sección anterior, este problema puede resolverse diseñando un estimador de estados para el sistema (40). Se propone el siguiente observador de tipo Luenberger

$$
\begin{aligned}
& \dot{\hat{z}}_{i}=\hat{z}_{i+1} \quad+L \dot{k}_{i}\left(z_{1}-\hat{z}_{1}\right), \quad(1 \leq i \leq \rho-1) \\
& \dot{z_{p}}=\hat{\eta}+\hat{\gamma}(\hat{z})+L^{\rho} \mathrm{K}_{\rho}\left(z_{1}-\hat{z}_{1}\right) \\
& \dot{\hat{\eta}}=\quad+L^{\rho+1} \kappa_{\rho+1}\left(z_{1}-\hat{z}_{1}\right)
\end{aligned}
$$

donde $\mathrm{L}>0$ y las constantes de estimación $\kappa_{\mathrm{i}}^{\prime}$ s son elegidas de tal manera que el polinomio $P_{p+1}(s)=s^{p+1}+\kappa_{p+1} s^{p}+\ldots+\kappa_{3} s^{2}+\kappa_{2} s+\kappa_{1}=0$ es Hurwitz.

Luego, a fin de disminuir el efecto de acciones de control con sobre disparo (las cuales pueden ser inducidas por el estimador de alta ganancia), la ley de control linealizante dada por la ecuación (41), puede modificarse como sigue

$$
u^{s}=\operatorname{Sat}\left\{\frac{1}{\hat{\gamma}(\hat{z})}\left[-\hat{\eta}+K^{T} \hat{z}\right]\right\}
$$

donde $u^{\mathrm{s}} \subset u \in \mathbb{R}, S a t\{\cdot\}: \mathbf{R} \rightarrow \mathscr{B} \subset \mathbf{R}$ es una función de saturación y $\mathscr{B}$ es un conjunto acotado. 
Nótese que la ley de control (44) nada más hace uso de estimados de las incertidumbres aglutinadas en $\Theta(z, v, u)$ (por medio de $\eta$ ) y valores estimados de los estados no medidos (a través de la salida $y=z_{1}$ ). Adicionalmente, notemos que una vez más el término incierto $\Xi(z, v, \eta, u)$ ha sido ignorado en el observador (43). Así pues,el CAR está dado por el compensador dinámico (43) y la ley de control (44).

El postulado que a continuación se presenta está basado fuertemente en los resultados de estabilización de sistemas linearizables por medio de retroalimentación de salida reportados en [19] y [28] (ver subsección 4.1.1).

Teorema 4.2: Asumamos que se satisfacen las suposiciones (H.4.1) - (H.4.4). Entonces, el controlador (43),(44) es un estabilizador semiglobal práctico robusto del sistema (36).

\subsubsection{A nálisis de estabilidad}

Sea $\varepsilon \in \mathbf{R}^{\rho+1}$ un vector de errores de estimación cuyos componentes están definidos por

$$
\begin{aligned}
\dot{\varepsilon}_{j} & =L^{p+1-j}\left(z_{1}-\hat{z}_{1}\right) ; 1 \leq j \leq \rho \\
\dot{\varepsilon}_{\rho+1} & =\eta-\hat{\eta}
\end{aligned}
$$

de manipulaciones algebráicas directas, no es dificil ver que

$$
\dot{\varepsilon}=L A(\kappa, r) \varepsilon+B \Phi_{2}(z, \eta, N(L) \varepsilon, u)
$$

donde

$$
A(\kappa, r)=\left[\begin{array}{ccccc}
-\kappa_{1} & 1 & 0 & \ldots & 0 \\
-\kappa_{2} & 0 & 1 & \ldots & 0 \\
\vdots & \vdots & \vdots & \ddots & \vdots \\
-\kappa_{\rho} & 0 & 0 & \ldots & 1 \\
-r \kappa_{\rho+1} & 0 & 0 & \ldots & 0
\end{array}\right]
$$

$\mathrm{B}=[0,0, \ldots, 0,1]^{\mathrm{T}}, \mathrm{N}(\mathrm{L})=\operatorname{diag}\left[\mathrm{L}^{-p}, \mathrm{~L}^{-\rho+1}, \ldots, \mathrm{L}^{-1}, 1\right]$ y $\Phi_{2}(\mathrm{z}, \eta, \mathrm{N}(\mathrm{L}) \varepsilon, u)$ es una función continua dada por

$$
\Phi_{2}(\cdot)=\sum_{k=1}^{\rho-1} z_{k+1} \partial_{z} \Theta+[\eta+\hat{\gamma} u] \partial_{\rho} \Theta+u^{s} \partial_{t} \delta+\zeta \partial_{v} \theta+\phi_{2} \operatorname{Sat}^{\prime}\{\cdot\}
$$

donde $S a t^{\prime}\{\cdot\}$ es la derivada de la función de saturación $S a t: \mathbb{R} \rightarrow \not B C \mathbb{R}$ y $r$ es un parámetro variante en tiempo dado por $r=[\hat{\gamma}(z)-\operatorname{Sat}\{\cdot\}(\gamma(z, v)-\hat{\gamma}(z))] / \hat{\gamma}(z)$. Asumiendo que $\operatorname{Sat}\{\cdot\}$ $\in[0,1]$, luego las suposiciones de $\hat{\gamma}(z) \geq \gamma(z, v)$ y $\operatorname{sgn}(\hat{\gamma}(z))=\operatorname{sgn}(\gamma(z, v))$ implican que $0<$ $r<1$ para todo $t \geq 0$. Nótese que si $\Phi_{2}(\cdot) \equiv 0$, el sistema (48) se reduce a la dinámica nominal 
del error de estimación dada por $\dot{\varepsilon}=\mathrm{LA}(\kappa, r) \varepsilon$. Si $0<r<1$ para todo $t \geq 0$, entonces $A(\kappa, r)$ es Hurwitz, por lo tanto la dinámica nominal es exponencialmente estable (para un bosquejo ver Apéndice A).

Por otra parte, si no existe incertidumbre asociada con la entrada de control (es decir, $\gamma(z)=\gamma(z, v))$, entonces $r=1$. Luego, para $L>0$ y la suposición que $P_{p+1}(s)$ es Hurwitz, el sistema de dinámica nominal $\varepsilon=\mathrm{LA}(\kappa, 1) \varepsilon$ es asintóticamente estable $(\varepsilon(\mathrm{t}) \rightarrow 0$ cuando $\mathrm{t} \rightarrow$ c) (ver Apéndice B).

Sin embargo, $r$ es un parámetro variante en tiempo por lo que probar estabilidad del sistema (46) no resulta obvio. Empero, a partir de un resultado reportado en [35] acerca del margen de estabilidad de sistemas lineales inciertos, podemos concluir que si todos los valores propios de $\mathrm{A}(\mathrm{K}, 1)$ están localizados alrrededor de -1 y $0<r<1$ para todo $\mathrm{t} \geq 0$, se garantiza la estabilidad global del sistema nominal $\dot{\varepsilon}=\mathrm{LA}(\kappa, r) \varepsilon$.

La condición $\operatorname{sgn}(\hat{\gamma}(z))=\operatorname{sgn}(\gamma(z, v))$ impuesta a la ganancia de alta frecuencia, $L$, fue usada para asegurar la estabilidad asintótica del sistema nominal de error de estimación, $\varepsilon \in$ $\mathbf{R}^{p+1}$. Notemos que si el signo de $\gamma(z, v)$ no es conocido a priori (lo cual está asociado con el hecho de conocer la 'direccionalidad' de las acciones de control), no se puede garantizar la estabilidad del sistema dinámico nominal $\dot{\varepsilon}=\mathrm{LA}(\kappa, r) \varepsilon$. Esta condición es fuerte. No obstante, es una hipótesis común en esquemas adaptativos de control [36]. Al margen de ello, afortunadamente para la mayoria de los sistemas químicos no es dificil conocer la direccionalidad de las acciones de control. Por ejemplo, se puede establecer si las acciones de control están destinadas a remover (reacción exotérmica) o añadir (reacción endotérmica) calor a un sistema reactante, o bien, se puede saber si se debe añadir concentración de tal o cual compuesto para desplazar el equilibrio químico hacia reactantes o productos [2].

Hasta aquí, únicamente se ha tratado la estabilidad del error nominal de estimación. Pero la dinámica de $\varepsilon \in \mathbb{R}^{p+1}$ está acoplada a la dinámica de sistema de estados $(z, v) \in \mathbb{R}^{n}$ como sigue

$$
\begin{aligned}
& \dot{z}_{j}=z_{j+1} ; \quad 1 \leq j \leq \rho-1 \\
& \dot{z}_{\rho}=\eta+\hat{\gamma}(z)\left[u^{s}(z, \eta)-u^{s}(z, \eta, N(L) \varepsilon)\right] \\
& \dot{v}=\zeta(z, v)
\end{aligned}
$$

donde $u^{\text {s }}$ significa versión saturada del control $u$. Es evidente que si $\varepsilon=0$ entonces el sistema (49) se reduce al sistema nominal controlado con retroalimentación de estado

$$
\begin{aligned}
& \dot{z}_{j}=z_{j+1} ; \quad 1 \leq j \leq \rho-1 \\
& \dot{z}_{\rho}=\eta+\hat{\gamma}(z) u^{s}(z, \eta) \\
& \dot{v}=\zeta(z, v)
\end{aligned}
$$


Por suposición el subsistema $\dot{v}=\zeta(z, v)$ es estable. También, $u^{s}(z, \eta)$ es una versión saturada de un control estabilizante por retroalimentación de salida definido globalmente. Ahora bien, el sistema nominal (50) puede ser estabilizado semiglobal y asintóticamente en un punto de equilibrio nominal sólo ajustando las cotas superior, $u^{\text {sup }}$, e inferior, $u^{\text {nf }}$, de la función $S a t: \mathbb{R} \rightarrow \not B \subset \mathbb{R}[19]$. Es decir, dado un conjunto compacto arbitrario de condiciones inciales, $I$, uno puede encontrar cotas superior, $u^{\text {sup }}$, e inferior, $u^{\text {inf }}$, de la función de saturación Sat: $\mathbf{R} \rightarrow \not \mathscr{B} \subset \mathbf{R}$ tal que $I$ está contenido en la región de atracción $\Omega$ del sistema.

Los argumentos anteriores muestran solamente que el sistema nominal $\dot{\varepsilon}=\operatorname{LA}(r) \varepsilon$ es globalmente asintóticamente estable mientras que el sistema (50) es semiglobalmente asintóticamente estable. Empero, el sistema a lazo cerrado está dado por los sistemas (46) y (49) donde las dinámicas de $(z, v) \in \mathbf{R}^{\mathrm{n}}$ y $\varepsilon \in \mathbf{R}^{\rho^{+1}}$ están acopladas. En este sentido, no hay un principio de separación para el sistema a lazo cerrado. Aunque la estabilidad el sistema total (46),(49) no puede ser determinada a partir de la estabilidad de los sistemas nominales $\dot{\varepsilon}=$ $\mathrm{LA}(r) \varepsilon$ y (49), por fortuna, recientes resultados teóricos (sobre la estabilidad de sistemas linealizables bajo acciones de retroalimentación de salida basada en observadores de alta ganancia [28]) permiten estatuir la establidad del sistema a lazo cerrado (46),(49).

A partir de la suposiciones se establece lo siguiente: (i) $u^{\mathrm{s}}(\mathbf{z}, \eta)$ y $u^{\mathrm{s}}(\hat{z}, \hat{\eta})$ son controladores globalmente acotados y, (ii) de la continuidad de $\Phi_{2}(z, \eta, N(L) \varepsilon, u)$ respecto a sus argumentos se tiene que

$$
\begin{aligned}
&\left|\hat{\gamma}(z)\left\{u^{s}(z, \eta)-u^{s}(z, \eta, N(L) \varepsilon)\right\}\right| \leq \delta_{1}(|\varepsilon|) \\
&\left|B \Phi_{2}(z ; \eta N(L) \varepsilon, v, u)\right| \leq \sigma_{1}+\sigma_{2}|\varepsilon|
\end{aligned}
$$

para todo $(z, \eta) \in \Gamma \subset \mathbb{R}^{\rho+1}, v \in \mathbb{R}^{\mathrm{n}-\rho}$ y $\varepsilon \in \mathbb{R}^{\rho+1}$, donde $\Gamma$ es un subconjunto compacto de $\mathbb{R}^{\rho+1}$, $\sigma_{1}$ y $\sigma_{2}$ son constantes positivas dadas y $\delta_{1}(|\varepsilon|)$ es una función continua acotada con $\delta(0)=$ 0 .

Usando el resultado de observación robusta [28] (ver Apéndice B), se puede concluir que las desigualdades $(51)$ implican que existe un número positivo $\mathrm{L}^{*}$ y unas $\operatorname{cotas} u^{\text {sup }}, u^{\text {inf }}$ tales que para un conjunto compacto de condiciones iniciales dado $I \subset \mathbf{R}^{\mathrm{n}+\rho}$ y todo $\mathrm{L}>\mathrm{L}^{*}, I$ está contenido dentro de la región de atracción $\Omega$ del sistema a lazo cerrado. El anterior resultado es conocido como estabilidad semiglobal asintótica del sistema a lazo cerrado.

\subsection{Linealización asintótica robusta de RCTA's exotérmicos}

Ahora considérese un RCTA exotérmico donde ocurre una reacción heterogénea: la oxidación catalizada de benceno a anhidrido maléico. El reactor en cuestión es uno del tipo de lecho fluidizado. La reacción procede sobre un catalizador de Molibdeno-Vanadio. Como motivación basta saber que el an. maléico es un insumo importante en la producción de: Resinas insaturadas de poliéster, aditivos para lubricantes, agroquímicos y copolímeros, por mencionar algunos productos químicos. 
Captulo cuarto

La oxidación de benceno a an. maléico es altamente exotérmica. Por ello, se requiere la remoción de calor mediante una chaqueta de enfriamiento. Para lograr la regulación de temperatura, en el RLF la chaqueta es alimentada mediante el bombeo de aire fresco.

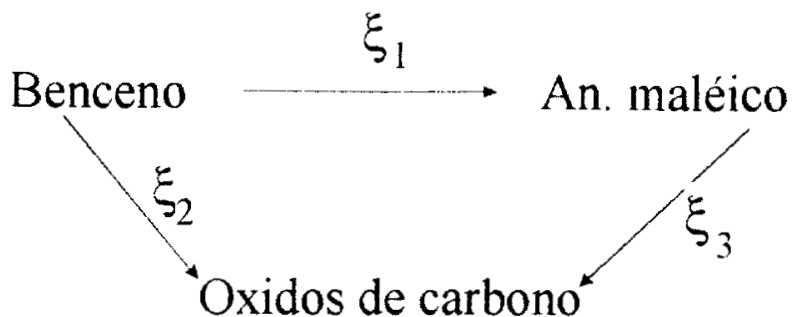

Figura 16. Esquema triangular de la oxidación de benceno

Un esquema de reacción triangular puede representar a la oxidación catalítica de benceno (ver Figura 16). Las expresiones de tasa cinética, para las reacciones del esquema de reacción triangular de oxidación de benceno, son de pseudo-primer orden. La dependencia de las tasas de reacción con la temperatura están dadas como sigue [37]

$$
R_{i}(x)=\xi_{i}(T) T_{R}, \quad i=1,2,3 .
$$

donde $\xi_{1}\left(T_{R}\right)=\xi_{i} \exp \left(-E a_{1} / R_{R}\right), E a_{1}$ es la energia de activación de la $i$-ésima reacción y $T_{R}$ es la temperatura del reactor. Cabe señalar que el concepto pseudo-primer orden en cinética química se utiliza para modelar tasas de conversión que se comportan como una de primer orden, lo cual no implica que lo sea. De hecho, las reacciones catalizadas pueden ser muy complejas y suelen involucrar fenómenos superficiales, de transferencia de masa, de desactivación, etc.

\subsubsection{Modelo dinámico del RLF}

El reactor puede ser modelado considerando lo siguente: (i) Modelo de dos fases (una, la fase densa, representa a las partículas sólidas y la otra, la fase burbuja, representa el gas que fluidiza al sólido), (ii) mezclado perfecto (favorecido por la agitación que provee la fase burbuja) y (iii) flujo tapón en la fase gaseosa (el gas no presenta retromezclado). Las consideraciones anteriores son hechas a partir de las bondades que ofrecen los reactores de lecho fluidizado. Luego de elaborar los balances de masa y energía y después de una redefinición de variables (para mayores detalles ver [37]) se tiene el siguiente modelo

$$
\begin{aligned}
& \dot{x}_{1}=a_{1}\left(d_{1}-x_{1}\right)+a_{2}\left(x_{2}-x_{3}\right)+a_{3}\left[\beta_{1} R_{1}(x)+\beta_{3} R_{3}(x)\right] x_{3}+x_{4} \beta_{2} R_{2}(x) \\
& \dot{x}_{2}=c_{1}+a_{2} b_{1}\left(x_{1}-x_{2}\right)+b_{2}\left(d_{2}-x_{2}\right) u \\
& \dot{x}_{3}=a_{a}\left(d_{3}-x_{3}\right)-a_{5}\left[R_{1}(x)+R_{3}(x)\right] x_{3} \\
& \dot{x}_{4}=a_{4}\left(d_{4}-x_{4}\right)+a_{5}\left[R_{1}(x) x_{3}-R_{3}(x) x_{4}\right]
\end{aligned}
$$


donde $x \in \mathbf{R}^{4}$ es un vector de estados cuyos componentes representan temperatua de reactor, temperatura de chaqueta, concentración de benceno y concentración de an. maléico, respectivemente. $a_{2}=a_{2}(\mathrm{t}), a_{2} b_{1}$ y $b_{2}=b_{2}(\mathrm{t})$ están asociados con los coeficientes de transferencia de calor del reactor, chaqueta y pared de reactor. $d_{1}=d_{1}(\mathrm{t}), d_{2}=d_{2}(\mathrm{t}), d_{3}=d_{3}(\mathrm{t})$ y $d_{4}=d_{4}(\mathrm{t})$ significan la temperatura de entrada al reactor, a la chaqueta y las concetraciones de entrada al reactor de benceno y an. maléico. $\beta_{1}=\beta_{1}(x), \beta_{2}=\beta_{2}(x)$ y $\beta_{3}=\beta_{1}(x)$ constituyen los términos asociados a la entalpía de reacción. $u$ es la tasa de flujo de aire a la chaqueta.

Los términos de cinética de reacción, $R_{i}(x)$ (con $\left.i=1,2,3\right)$, son expresiones de tipo Arrhenius (es decir, obedecen a las propiedades (P.1) y (P.2), ver primer capítulo). Sin embargo, tales expresiones no se conocen con exactitud. Por ejemplo, se desconoce como afecta la desactivación del catalizador a la conversión. O bien, se ignora el desgaste del catalizador debido a la fricción que provoca su residencia en el lecho fluidizado, y cómo este fenómeno afecta la conversión. Más aún, el modelo cinético asume que las tasas de reacción tienen comportamiento similar a las de primer orden (respecto a la concentración de la sustancia que se convierte), empero, esto no significa que necesariamente sea de primer orden. Además, no se incluye la influencia del catalizador en dichos términos cinéticos.

En lo que respecta a las perturbaciones exógenas, éstas son inducidas en el reactor por los flujos de alimentación $d_{\mathrm{j}}, \mathrm{j}=1,2,3,4$. De las cuales, se pueden tener mediciones en línea (con bajo costo) de las temperaturas que entran tanto al reactor como a la chaqueta. Pero, tener acceso a mediciones de las concentraciones de entrada de benceno y an. maléico puede inducir retardo en su medición (aún cuando se haga usando cromatógrafos de gases rápidos) y además aumenta el costo de operación del controlador.

\subsubsection{La regulación robusta de temperatura del $R L F$}

Sea $z_{1}=x_{1}-x_{1}^{*}, y=z_{1}$, entonces el grado relativo del sistema (53) es $\rho=2$. Luego, el sistema (53) puede ser transformado a la forma canónica (35) mediante el siguiente cambio de coordenadas: $z_{1}=x_{1}-x_{1}^{*}, z_{2}=\dot{y}$. Con lo cual el sistema (53) puede ser reescrito como

$$
\begin{aligned}
& \dot{z}_{1}=z_{2} \\
& \dot{z}_{2}=\alpha(z, v)+\gamma(z, v) u \\
& \dot{v}=\zeta(z, v) \\
& y=z_{1}
\end{aligned}
$$

donde $v=\left[\left(x_{3}-x_{3}^{*}\right),\left(x_{4}-x_{4}^{*}\right)\right] \in \mathbb{R}^{2}$ es un vector de estados no observables, $\alpha(z, v)=\mathscr{Q}_{f}^{2} h(x)=$ $a_{1} \dot{d}+\dot{a}_{2}\left(x_{2}-\mathrm{z}_{2}\right)-\left(a_{1}-a_{2}\right) \mathrm{z}_{2}+a_{2}\left[\mathrm{c}_{1}+a_{2} b_{2}\left(\mathrm{z}_{1}-x_{2}\right)+a_{3}\left\{\left[\beta_{1} \partial R_{1}\left(\mathrm{z}_{1}\right) / \partial \mathrm{z}_{1}+\beta_{2} \partial R_{3}\left(\mathrm{z}_{1}\right) / \partial \mathrm{z}_{1}+\right.\right.\right.$ $\left.\left.\beta_{3} \partial R\left(z_{1}\right) / \partial z_{1}\right] z_{2}+\left[\beta_{1} R_{1}\left(z_{1}\right)+\beta_{2} R_{3}\left(z_{1}\right)+\beta_{3} R_{2}\left(z_{1}\right) v_{2}\right]\left[a_{4}\left(d_{3}-v_{1}\right)-a_{5}\left(R_{1}\left(z_{1}\right)+R_{3}\left(z_{1}\right)\right) v_{2}\right]\right\}$ y $\gamma(z, v)=\mathscr{L}_{g} \mathscr{L}_{f} h(x)=a_{2} b_{2}\left(d-x_{2}\right) u$. Nótese que la incertidumbre asociada con la entrada de control, $\gamma(z, v)$, representa a la transferencia de calor entre el reactor y la chaqueta. 
Capitulo cuarto

Proposición 4.3: El sistema (54) es fase minima. Es decir, el subsistema (54.2) es estable.

Pnueba: De acuerdo con la transformación de coordenadas, los estados no observables están dados como $v_{1}=x_{3}-x_{3}^{*}, v_{2}=x_{4}-x_{4}^{*}$. Entonces, la dinámica cero del $\operatorname{RLF}\left(z_{1}=0, z_{2}\right.$ $=0$ ) es

$$
\begin{aligned}
& \dot{v}_{1}=-\left\{a_{4}+a_{5}\left[R_{1}\left(x_{1}^{*}\right)-R_{3}\left(x_{1}^{*}\right)\right]\right\} v_{1} \\
& \dot{v}_{2}=a_{5} R_{1}\left(x_{1}^{*}\right) v_{1}-\left[a_{4}-R_{2}\left(x_{1}^{*}\right)\right] v_{2}
\end{aligned}
$$

donde $R_{i}\left(x_{1}^{*}\right)$ es la tasa cinética dada por la expresión de tipo Arrhenius (ecuación (52)). Entonces es fácil verificar que las ecuaciones de la dinámica cero son exponencialmente estables, por lo tanto el sistema (54) es de fase mínima.

Aqui es pertinente hacer los siguientes comentarios acerca de las suposiciones: La hipótesis (H.4.1) es práctica puesto que actualmente es posible llevar a cabo mediciones de temperatura con tasas de muestreo del orden de $1000 \mathrm{~Hz}$. Además, estas mediciones no presentan retardo en medición y son poco costosas. Adicionalmente, asúmase que la temperatura de chaqueta, $x_{2}, y$ la temperatura de alimentación, $d_{2}$, están disponibles para retroalimentación por medición en línea.

En lo referente a (H.4.2) y (H.4.3), se puede proclamar que éstas son realistas. Por un lado, las hipótesis hechas para modelar la cinética de reacción (como asumir que el comportamiento es de primer orden) y la falta de conocimiento acerca de fenómenos involucrados en la oxidación catalítica de benceno son argumentos suficientes para justificar que la tasa de conversión no está disponible para retroalimentación. Lo anterior implica que: Si las tasa de conversión no están disponibles para retroalimentación, es decir son términos inciertos, la transformación de coordenadas (aunque invertible) depende de funciones inciertas, por consiguiente, $\alpha(z, v)$ y $\gamma(z, v)$ son funciones desconocidas. Además, los coeficientes de transferencia de calor ( $y$ su variación debida a incrustaciones, ensuciamiento, etc.) es desconocida. Por otro lado, dado que la oxidación de benceno en altamente exotérmica, mientras ésta esté llevándose a cabo, la diferencia entre la temperatura de alimentación y la del reactor, $\left(d-x_{2}\right)$, será diferente de cero. Esto tiene dos implicaciones: (a) Se conoce la direccionalidad de las acciones de control (es decir, se sabe que hay remoción de calor mediante la chaqueta), esto significa que se conoce el signo de $\gamma(z, v)$ y (b) El grado relativo del sistema está bien definido para todo $t \geq 0$. Adicionalmente, a partir de información del diseño del equipo (materiales utilizados, dimensiones, etc.), se puede disponer de valores nominales de los coeficientes de tranferencia de calor.

Por último, y en lo que concierne a (H.4.4), se ha probado que el modelo del RLF es de fase mínima (Proposición 4.3).

En concordancia con los argumentos anteriores, existe un $\hat{\gamma}(z)$ tal que $\operatorname{sgn}(\hat{\gamma}(z))=$ $\operatorname{sgn}(\gamma(z, v))$. Luego, definiendo las siguientes variables $\delta(z, v)=\gamma(z, v)-\hat{\gamma}(z), \Theta(z, v, u)=\alpha(z, v)$ $+\delta(z, v) u$ y $\eta=\Theta(z, v, u)$, donde $\gamma(z)=\hat{a}_{2} b_{2}\left(d_{2}-x_{2}\right)$. El sistema (53) puede ser escrito como sigue 


$$
\left.\begin{array}{c}
\dot{z}_{1}=z_{2} \\
\dot{z}_{2}=\eta+\hat{\gamma}(z) u \\
\dot{\eta}=\Xi(z, v, \eta, u) \\
\dot{v}=\zeta(z, v) \\
y=z_{1}
\end{array}\right\}
$$

La dinámica de los estados del subsistema (55.1) puede ser reconstruida a partir de la salida $y=z_{1}$ mediante un observador de tipo Luenberger. De esta manera, se puede proveer con un valor estimado de la incetidumbres a una ley de control linealizante. Y dado que el valor estimado de $(z, \eta)$ es obtenido por un observador de alta ganancia (sistema (42)), se puede utilizar una función de saturación para disminuir el efecto del fenómeno de sobredisparo en la salida del reactor. Así, el controlador resultante está dado por

$$
\begin{aligned}
& \dot{\hat{z}}_{1}=\hat{z}_{2} \quad+\operatorname{Lx}_{1}\left(z_{1}-\hat{z}_{1}\right) \\
& \left.\dot{z}_{2}=\hat{\eta}+\hat{\gamma}(\hat{z}) u+L^{2} x_{2}\left(z_{1}-\hat{z}_{1}\right)\right\} \\
& \dot{\hat{\eta}}=\quad+L^{3} k_{3}\left(z_{1}-\hat{z}_{1}\right) \\
& u=\operatorname{Sat}\left\{\frac{1}{\hat{\gamma}(\hat{z})}\left[-\hat{\eta}+K_{1} \hat{z}_{2}+K_{2} \hat{z}_{1}\right]\right\}
\end{aligned}
$$

donde $\mathrm{L} \geq \mathrm{L}^{*}>0$ y las constantes de estimación, $\kappa_{i}^{\prime} \mathrm{s}$, son elegidas tales que el polinomio característico de

$$
A(r)=\left[\begin{array}{lll}
-k_{1} & 1 & 0 \\
-\kappa_{2} & 0 & 1 \\
-r \kappa_{3} & 0 & 0
\end{array}\right]
$$

(dado por $s^{3}+\kappa_{1} s^{2}+\kappa_{2} s+\left(\kappa_{3}+r\right)=0$ ) es Hurwitz y $0<r<1$. Así mismo, las ganancias de control se eligen tales que $s^{2}+K_{1} s+K_{2}=0$ tiene todas sus raíces localizadas en el hemiplano abierto izquierdo del plano complejo. Entonces, de acuerdo con los resultados de la subsección 4.2.1, el sistema a lazo cerrado es estable.

Cabe recordar que el parámetro $r=\left[\hat{\gamma}(z)-S a t^{\prime}\{\cdot\}(\gamma(z, v)-\hat{\gamma}(z))\right] / \hat{\gamma}(z)$, puede ser interpretado como un parámetro variante en tiempo y expresa la relación entre las incertidumbres asociadas a la entrada de control y el valor estimado de éstas. Esto implica que si los coeficientes de transferencia de calor son exactamente conocidos (es decir, no hay 
Capitulo cuarto

incertidumbre asociada con la entrada de control $), \gamma(z, v) \cong \gamma(z)$ y por lo tanto, $r \approx 1$ para todo $t \geq 0$. Esto, en aplicaciones prácticas, es posible obtener una expresión heurística del ensuciamiento del sistema de remoción de calor (lo cual es común en la programación de mantenimiento preventivo de equipo industrial).

\subsubsection{El desempeño del CAR en la regulación de temperatura}

Para evaluar el desempeño del controlador (56), se tomaron los valores de los parámetros de modelo reportados en [37]. La temperatura de operación del RLF es $\mathrm{T}_{\mathrm{r}}=$ $733.33 \mathrm{~K}$, para la cual el estado estacionario del reactor es inestable [37]. Las condiciones iniciales son: $\mathrm{T}_{\mathrm{r}}^{0}=703 \mathrm{~K}, \mathrm{~T}_{\mathrm{u}}^{0}=303 \mathrm{~K}, \mathrm{x}_{\mathrm{b} z}^{0}=0.02 \mathrm{y} \mathrm{x}_{\mathrm{um}}^{0}=0.0$, donde $\mathrm{T}_{\mathrm{r}}^{0}, \mathrm{~T}_{\mathrm{c}}^{0}, \mathrm{x}_{\mathrm{am}}^{0}$ y $\mathrm{x}_{\mathrm{b},}^{0}$ son las temperaturas del reactor, de chaqueta, fracción mol de benceno y fracción mol de an. maléico, respectivamente.

Los valores de las ganancias de control son: $\mathrm{K}_{1}=\mathrm{K}_{2}=0.08$. El parámetro de estimación $\mathrm{L}=0.45$. Los valores de las constantes de estimación, $\kappa_{i}^{\prime} \mathrm{s}$, son elegidos tales que la matriz compañera $A(k, 1)$ (ver ecuación (57)) tiene todos sus autovalores localizados en -1 . Las cotas superior e inferior de la señal de retroalimentación son: $u^{\text {sup }}=1.0$ y $u^{\text {inf }}=0.1$. Cabe señalar que la saturación de las acciones de control están asociadas a la capacidad del actuador.

A fin de simular el sistema a lazo cerrado, supóngase que los valores estimados de los coeficientes de transferencia de calor tienen una desviación de entre un 10 y $15 \%$ con respecto a los valores actuales. Arbitrariamente, consideremos que $\hat{a}_{2}=1.1 a_{2}=1.54 \times 10^{-3} \mathrm{y}$ $b_{2}=0.85 b_{2}=0.118$. Sometamos al RLF a lo siguiente: (i) Cambios de referencia, (ii) perturbaciones exógenas (representadas por: mediciones ruidosas, fluctuaciones en la composición de reactante alimentado y cambios drásticos en la transferencia de calor).

La temperatura del RLF se regula en $T_{r}=733.33 \mathrm{~K}$ (para la cual, como ya se dijo, el estado estacionario es inestable). Luego, en $\mathrm{t}=200 \mathrm{~s}$, se regula la temperatura en $720 \mathrm{~K}$. Además, en $\mathrm{t}=100 \mathrm{~s}$, se induce un cambio de $-15 \%$ en el coeficiente de transferencia de calor de la pared del reactor (los valores de $\hat{a}_{2}$ y $b_{2}$ se mantuvieron constantes con lo que su desviación se modifica). Adicionalmente, en $\mathrm{t}=300 \mathrm{~s}$, se induce una perturbación periódica en la concentración de alimentación de benceno, $d_{3}=d_{3}^{*}+0.1 \operatorname{sen}(\omega)$, donde $d_{3}^{*}=1.0$ y $\omega=$ $0.5 \mathrm{~Hz}$.

En la Figura 17 se presenta el desempeño del CAR. La Figura 17.a contiene la respuesta de la temperatura del reactor mientras que en la Figura 17.b se muestra la dinámica de la ley de control. Puede observarse que el CAR estabiliza al reactor en el punto de operación inestable. Además tiene buenas propiedades de rechazo a perturbaciones en la alimentación y en la transferencia de calor. Sin embargo, aunque con una respuesta aceptable en la salida del RLF, cuando se inducen cambios de referencia las acciones de control se saturan unos instantes. Otro punto a señalar es que cuando se disminuye la transferencia de calor de la pared del reactor, se requiere más flujo de enfriante para remover la cantidad de calor necesaria para regular la salida (ver Figura 17.b). Esto cumple con los conocimientos 
más elementales de la transferencia de calor.
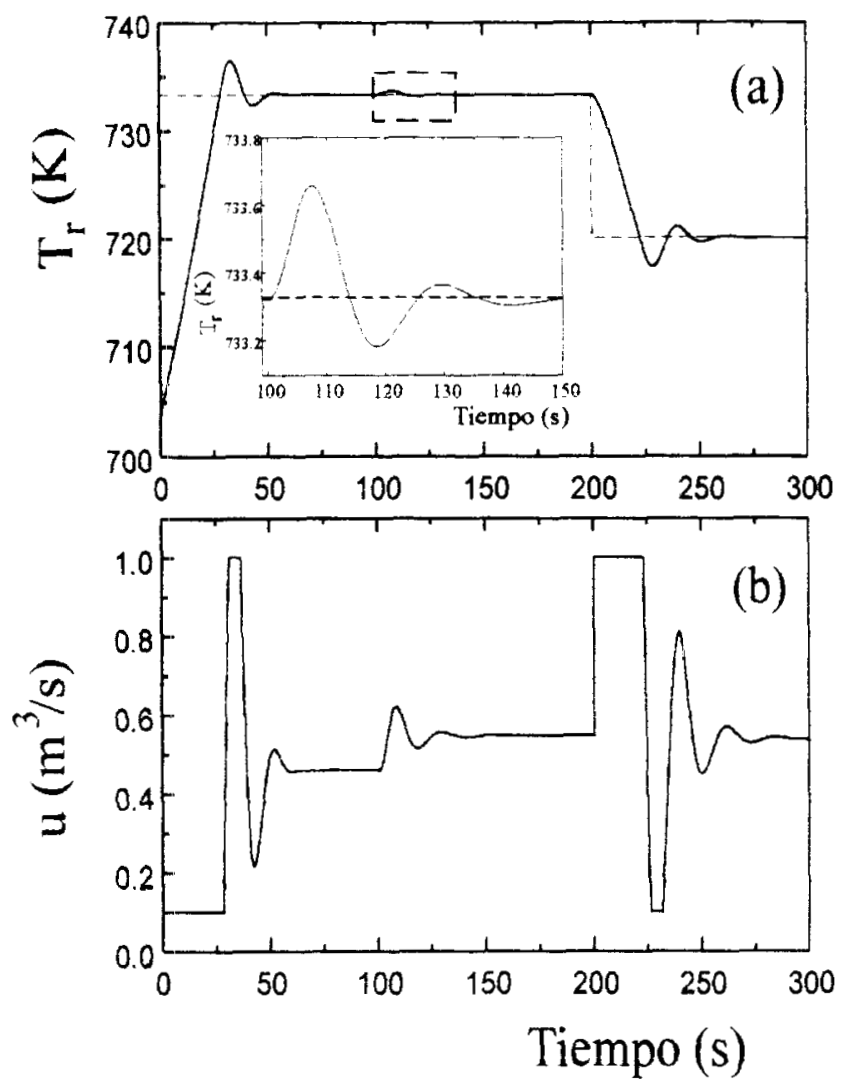

Figura 17. Respuesta del CAR en la regulación de temperatura en un reactor exotérmico

Ahora consideremos otra clase de perturbación exógena. Supóngase que la mediciones están contaminadas con ruido aditivo, i.e. $y=z_{1}+s_{n}$. La señal de ruido, $s_{n}$, puede ser simulada con la generación de números aleatorios. Para hacer realista el efecto de $s_{n}$, asumamos que se tiene un error de medición de $\pm 3 \mathrm{~K}$, lo cual implica que la amplitud de $s_{n}$ es de $0.5 \%$, es decir, $\mathrm{s}_{\mathrm{n}}=a_{\mathrm{n}} \mathrm{N}_{\mathrm{a}}$, donde $a_{\mathrm{n}}=0.005$ y $\mathrm{N}_{\mathrm{a}} \in[-1,1]$ es un número aleatorio. Cabe recordar que el modelo (42) del RLF es un modelo normalizado adimensional.

Con el fin de probar el efecto combinado de saturación y medición ruidosa, considérese un cambio de referencia de mayor magnitud que el inducido en la simulación anterior. Primero se regula la temperatura del reactor, $T_{r}=733.33 \mathrm{~K}$. Luego, a $t=100 \mathrm{~s}$ se induce un cambio de referencia de $-25 \mathrm{~K}$.

En la Figura 18 se muestra el desempeño del CAR ante cambio de referencia y en presencia del ruido aditivo simulado. Aunque el CAR no fue diseñado para controlar a pesar de mediciones ruidosas, sin embargo para la regulación de temperatura del RLF es satisfactoria (Figura 18.a) y el controlador tiene un desempeño aceptable (Figura 18.b). 
No obstante, se debe tener cuidado con esta aseveración. Puesto que el parámetro de alta ganancia, L, permite reconstruir señal de alta frecuencia (ver sección 3.3), si $\mathrm{L}$ » $\mathrm{L}^{*}>1$, la señal de ruido se amplifica y el sistema a lazo cerrado puede desestabilizarse. En particular, para la estabilización del RLF se requiere un valor del parámetro de alta ganancia pequeño, $\mathrm{L}=0.45 \mathrm{~Hz}$, así que señales de alta frecuencia (como las de ruido) son filtradas por el observador de Luenberger.

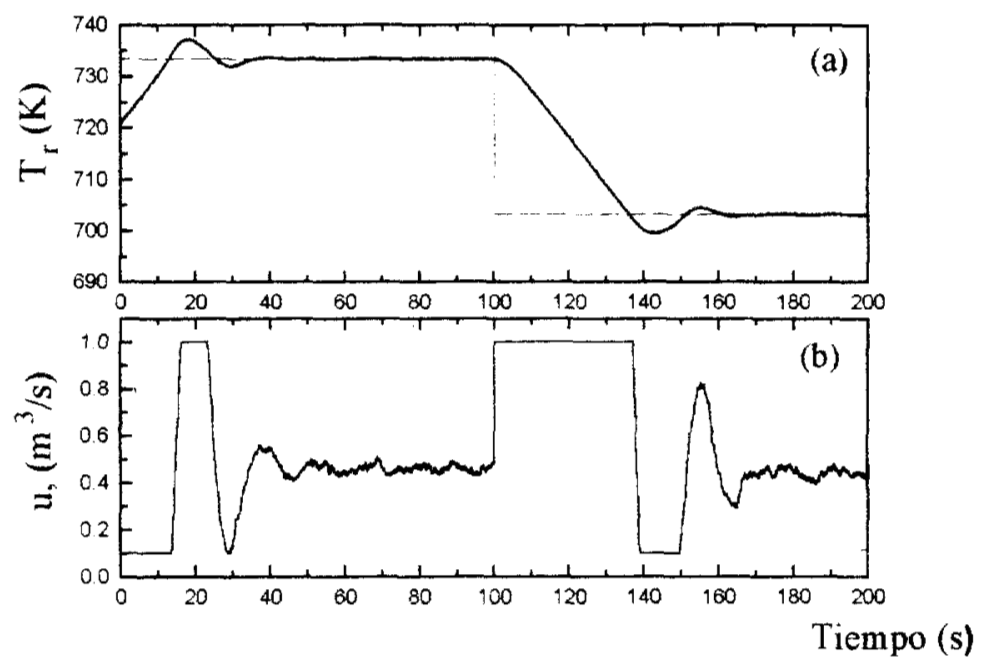

Figura 18. Desempeño del CAR en presencia de mediciones ruidosas

\subsection{Regulación de sustrato en una clase de fermentador}

Consideremos ahora una clase de reactor biológico, un proceso de lote alimentado. El biorreactor es un fermentador para la obtención de levadura para el uso en la elaboración de pan (Baker's yeast). En términos cualitativos, el objetivo de la regulación de sustrato, en esta clase de fermentador, consiste en determinar la concentración de sustrato alimentado al biorreactor para que la producción de biomasa sea la mayor posible en un intervalo de tiempo $\mathrm{t} \in\left[0, \mathrm{t}^{*}\right]$, asegurando que la concentración de etanol se mantenga por debajo de un valor crítico dado. El $\mathrm{CH}_{2} \mathrm{CH}_{3} \mathrm{OH}$ es producido por los microorganismos al consumir susrato. Si se logra mantener la concentración de alcohol por debajo de su valor crítico, la levadura puede aprovechar esta sustancia para aumentar su población mientras que por encima de la concetración crítica se inhibe el crecimiento de biomasa. En otras palabras, el alcohol etílico puede deteriorar el producto final si sobrepasa una concentración crítica. Para matener la concentración de $\mathrm{C}_{2} \mathrm{H}_{5} \mathrm{OH}$ en un nivel inferior de su valor crítico se debe regular la concentración de sustrato que entra al biorreactor.

El problema de control no resulta fácil debido a: (i) La presencia de incertidumbres, Las incertidumbres están asociadas a la carencia en el conocimiento referente a la tasa de formación y consumo de etanol. (ii) la naturaleza nolineal del proceso y, (iii) las variaciones 
parametricas involucradas.

A fin de formular correctamente el problema de control, es pertinente recordar las siguientes características del fermentador en cuestión [27]: (a) Sean $\xi_{1}(x)$ y $\xi_{2}(x)$ las tasas de consumo y formación de etanol, respectivamente. Esto implica que $\xi_{\mathrm{i}}(x) \geq 0, \mathrm{i}=1,2$. (b) $\mathrm{Si}$ la concentración de sustrato $x_{\mathrm{s}} \leq x_{\mathrm{s}}^{\mathrm{c}}$ (donde $x_{\mathrm{s}}^{\mathrm{c}}$ se conoce como la concentración crítica de sustrato) no existe formación de etanol, $\xi_{2}(x)=0$. (c) En esta fermentación, los microorganismos pueden consumir o producir etanol, pero no pueden hacer ambas cosas a la vez. Es decir, $\xi_{1}(x)$ y $\xi_{2}(x)$ son mutuamente excluyentes, o sea, $\xi_{1}(x)>0 \Rightarrow \xi_{2}(x)=0$ y viceversa. (d) La formación de etanol inicia si $x_{\mathrm{s}}>x_{\mathrm{s}}^{\mathrm{c}}$. Es decir, si $x_{\mathrm{s}}<x_{\mathrm{s}}^{\mathrm{c}}$ entonces $\xi_{1}(x)>$ 0 . En otras palabras, si existe etanol presente en el biorreactor y $x_{\mathrm{s}}<x_{\mathrm{s}}^{\mathrm{c}}$, el $\mathrm{C}_{2} \mathrm{H}_{5} \mathrm{OH}$ es consumido por la levadura. (e) Para el caso tratado aquí, se ha reportado que $x_{\mathrm{s}}^{\mathrm{c}}=0.28 \mathrm{~g} / \mathrm{L}$ [27].

\subsubsection{El modelo dinámico del fermentador}

El modelo dinámico de la fermentación de levadura para pan usada en esta sección es esencialmente el mismo que el descrito en [27] y está dado por

$$
\begin{aligned}
& \dot{x}_{1}=\left[\mu(x)-\frac{u}{x_{5}}+0.48 \xi_{1}(x)\right] x_{1} \\
& \dot{x}_{2}=-\left[Y_{s} \mu(x)+k_{m}+\xi_{2}(x)\right] x_{2}+\left[s_{e}-x_{2}\right] \frac{u}{x_{5}} \\
& \dot{x}_{3}=\left[2.3 \times 10^{-3}+7 \times 10^{-3} \mu(x)\right] x_{1}-x_{3} \frac{u}{x_{5}} \\
& \dot{x}_{4}=\left[\xi_{2}(x)-\xi_{1}(x)\right] x_{1}-x_{4} \frac{u}{x_{5}} \\
& \dot{x}_{5}=u \\
& y=x_{2}
\end{aligned}
$$

donde $x \in \mathbf{R}^{5}$ es un vector de estados cuyos elementos significan la concentración de biomasa, de sustrato, de sustancia inhibitoria (distinta a etanol), de alcohol etílico y volumen del caldo de fermentación, respectivamente. $\xi_{1}(x)$ es la tasa de consumo de etanol mientras que $\xi_{2}(x)$ es la de formación. $\mu(x)$ es la tasa específica de crecimiento de biomasa. La entrada de control, $u$, es el flujo de alimentación de sustrato (tasa de dilución) el cual está restringido a estar en el conjunto compacto $\mathscr{P}_{u}=\left\{u\right.$ : $\left.0<u^{\text {inf }} \leq u \leq u^{\text {sup }}\right\}$. Con lo anterior la saturación de la acción de control cobra un sentido físico (desde el punto de vista de los requerimientos intrínsecos del proceso) [27]. Finalmente, $s_{\mathrm{e}}=\mathrm{s}_{\mathrm{e}}(\mathrm{t})$ es la concentración de sustrato alimentado al biorreactor, la cual es una fuente de posibles perturbaciones exógenas. 
Capitulo cuarto

4.t. 2 El diseño del controlador robusto para regulación de sustrato

Sea $z=x_{z}-x_{2}^{*}$ y $y=z$. Tomando la primer derivada de $y=z$, se tiene que el sistema (57) tiene grado relativo $\rho=1$. Luego, el sistema (57) puede ser reescrito en la forma canónica (35) como sigue

$$
\begin{aligned}
& \dot{z}=\alpha(z, v)+\gamma(z, v) u \\
& \dot{v}=\zeta(z, v) \\
& y=z
\end{aligned}
$$

donde $\alpha(z, v)=-\left[Y_{s} \mu(z, v)+k_{m}+\xi_{2}(z, v)\right] v_{1}, \gamma(z, v)=\left(s_{e}-z\right) / v_{4}, v=\left(v_{1}, v_{2}, v_{3}, v_{4}\right)^{\mathrm{T}}=\left(x_{1}, x_{3}, x_{4}\right)^{T}$ es un vector que representa la dinámica interna. Obsérverse que, en este caso, las incertidumbres asociadas con la entrada de control están representadas por la señal no medida, $\mathrm{s}_{\mathrm{e}}$, $\mathrm{y}$ el estado no observado que significa el volumen del caldo de cultivo, $v_{4}$.

Proposición 4.4: El sistema (58) es de fase mínima. Es decir, en $z=0$ la dinámica interna $\dot{v}=\zeta(z, v)$ es estable.

Pnieba: Sea $z=0$ entonces $x_{2}=x_{2}^{*}$. Luego la dinámica interna está dada por

$$
\begin{aligned}
& \dot{v}_{1}=\left[\mu\left(v ; x_{2}^{*}\right)-\frac{u}{v_{5}}+0.48 \xi_{2}\left(v ; x_{2}^{*}\right)\right] v_{1} \\
& \dot{v}_{2}=\left[2.3 \times 10^{-3}+7 \times 10^{-3} \mu\left(v ; x_{2}^{*}\right)\right] v_{1}-v_{2} \frac{u}{v_{4}} \\
& \dot{v}_{3}=\left[\xi_{2}\left(v ; x_{2}^{*}\right)-\xi_{1}\left(v ; x_{2}^{*}\right)\right] v_{1}-v_{3} \frac{u}{v_{4}} \\
& \dot{v}_{4}=u
\end{aligned}
$$

Por un lado, para analizar la estabilidad del sistema (59), asúmase que la tasa especifica de crecimiento de biomasa es sensible a la concentración de la sustancia inhibitoria y está dada por [27]: $\mu\left(v ; x_{2}^{*}\right)=\left[\mu_{\mathrm{M}} x_{2}^{*} /\left(\mathrm{K}_{\mathrm{M}}+x_{2}^{*}\right)\right]\left[1 /\left(1+v_{2}\right)\right]$. Por otro lado, con el mismo fin, consideremos las expresiones heurísticas para las tasas de consumo y formación de etanol reportadas en [38] las cuales capturan sus principales características. Así se tiene que: $\xi_{2}\left(v ; x_{2}^{*}\right)$ $=0.138-0.062 v_{2}+2.8 \times 10^{-3} /\left(x_{2}^{*}-0.28\right)$ y $\xi_{1}\left(v ; x_{2}^{*}\right)=0.0155+0.123 \ln \left(x_{2}^{*}\right)$, donde $0<x_{2}^{*} \leq x_{2}^{*}$. La acción de control está acotada puesto que $u \in \mathscr{F}_{u}=\left\{u: 0<u^{\text {inf }} \leq u \leq u^{\text {sup }}\right\}$, donde $\mathscr{\mathscr { F }}_{u} \subset$ es un conjunto compacto. Combinando las expresiones anteriores y el sistema (59), es fácil ver que la dinámica interna es asintóticamente globalmente estable. Por lo tanto, el sistema (58) es de fase mínima. 
Es conveniente hacer algunos comentarios concernientes a las hipótesis planteadas y su implicación en el fermentador de lote alimentado. Como ya se ha mencionado, en biorreactores es posible hacer mediciones de sustrato con rapidez suficiente para asegurar que se dispone de estas medidas para retroalimentación. En cuanto a (H.4.2), está claro que $\left(\mathrm{s}_{\mathrm{e}}\right.$ z) $/ v_{4}$ es diferente de cero y dado que $v_{4} \in\left(0, v_{4, \max }\right.$ ] donde $v_{4, \max }<x$ es el volumen total del reactor, entonces $\gamma(\mathbf{z}, v)$ está acotada. (H.4.3) es una función realista. Dado que la tasa específica de crecimiento de biomasa, $\mu(x)$ y la de consumo de etanol son desconocidas, $\alpha(z, v)$ también es desconocida. Por otra parte, no se dispone de mediciones de $s_{\mathrm{e}}$ ni del estado no observable, $v_{4}$. por lo tanto, $\gamma(z, v)$ es una función incierta. No obstante, se pueden conocer valores nominales tanto de $s_{\mathrm{e}}$ como de $v_{4}$. En lo que respecta a (H.4.4) se ha probado que el sistema (59) es de fase minima (Proposición 4.4).

Sea $\delta(z, v)=\gamma(z, v)-\hat{\gamma}(z)$, donde $\hat{\gamma}(z)=\left(s_{e}^{*}-z\right) / v_{4}^{*}\left(s_{e}^{*}\right.$ y $v_{4}^{*}$ son valores nominales de la concentración de sustrato a la entrada y el volumen del caldo de cultivo, respectivamente), $\Theta(z, v, u)=\alpha(z, v)+\delta(z, v) u$ y $\eta=\Theta(z, v, u)$. Así que el sistema (59) puede ser reescrito en la siguiente forma equivalente

$$
\begin{aligned}
& \dot{z}=\eta+\hat{\gamma}(z) u \\
& \dot{\eta}=\Xi(z, v, \eta u) \\
& \dot{v}=\zeta(z, v) \\
& y=z
\end{aligned}
$$

donde $\Xi(z, v, \eta, u)=-Y_{s}[\eta+\hat{\gamma}(z) u]\left[\partial_{z} \mu(z, v)+\partial_{z} \xi_{2}(z, v)+u \partial_{z} \gamma(z, v)+\partial_{z} \gamma(z)\right]-Y_{s}\left[\partial_{1} \mu(z, v)+\right.$ $\left.\hat{C}_{1} \xi_{2}(z, v)+u \partial_{i} \gamma(z, v)\right] \dot{v}_{i}+(\gamma(z, v)-\hat{\gamma}(z)) \dot{u}, \mathrm{i}=1,2,3,4$.

De nuevo, para la aplicación práctica no es indispensable obtener el sistema (61). Este sólo será usado para el análisis de estabilidad a lazo cerrado. Una vez más, las incertidumbres han sido aglutinadas en una función no lineal la cual es interpretada como la variable de estado $\eta$. La dinámica de los estados $(z, \eta)$ del sistema (61) puede ser reconstruida a partir de mediciones, nuevamente se propone el siguiente observador de tipo Luenberger.

$$
\begin{aligned}
& \dot{z}=\eta+\hat{\gamma}(\hat{z})+L \kappa_{1}(z-\hat{z}) \\
& \dot{\hat{\eta}}=\quad+L^{2} k_{2}(z-\hat{z})
\end{aligned}
$$

donde $(\hat{z}, \eta)$ son valores estimados de $(z, \eta)$, respectivamente. $L \geq L^{*}>0$ es el parámetro de estimación de alta ganancia, las constantes $\kappa_{i}^{\prime}$ s son elegidas tales que el polinomio $s^{2}+\kappa_{2} s$ $+\kappa_{1}=0$ es Hurwitz. Entonces, la ley de control está dada por: $\boldsymbol{u}=v_{4}^{*}\left[-\hat{\eta}+\mathbf{K}_{1} \hat{z}\right] /\left(\mathbf{s}_{\mathrm{e}}^{*}-\hat{z}\right)$. Para disminuir y prevenir los efectos del posible sobredisparo en la salida que llegaran a ser inducidos por el estimador de alta ganancia, la ley de control anterior, puede ser modificada mediante una función de saturación. Así, la versión saturada de la ley de control linealizante está dada por 
Capitulo euarto

$$
u=\operatorname{Sat}\left\{\frac{v_{4}^{*}}{s_{e}^{*}-\hat{z}}\left[-\hat{\eta}+K_{1} \hat{z}\right]\right\}
$$

donde $S a t\{\cdot\}: \mathbb{R} \rightarrow \not B \subset \mathbb{R}, \mathscr{B}$ es un conjunto acotado. En particular, para el fermentador (58), la tasa de dilución, $u$, está restringida a permanecer en el conjunto compacto $\mathscr{\mathscr { P }}=\left\{u: 0<u^{\text {inf }}\right.$ $\left.\leq u \leq u^{\text {sup }}\right\}$. Por consiguiente, Sat $\{\cdot\}=0.1 \mathrm{~L} / \mathrm{Hr}$ si $u \leq u^{\text {inf }}=0.1 \mathrm{~L} / \mathrm{Hr}$, Sat $\{\cdot\}=10.0$ si $u \leq$ $u^{\text {sup }}=10.0 \mathrm{~L} / \mathrm{Hr}$ y Sat $\{\cdot\}=u$ en cualquier otro caso

\subsubsection{La implementación numérica}

A fin de llevar a cabo la implementación del controlador (62),(63). Supóngase que la tasa específica de crecimiento de biomasa es sensible a la concentración de la sustancia inhibitoria y está dada por [27]

$$
\mu\left(v ; x_{2}^{*}\right)=\left[\mu_{\mathrm{M}} x_{2}^{*} /\left(\mathrm{K}_{\mathrm{M}}+x_{2}^{*}\right)\right]\left[1 /\left(1+v_{2}\right)\right]
$$

Además, asúmase que las expresiones para las tasas de consumo y formación de etanol reportadas están dadas por las expresiones heurísticas reportadas en [38]. Así se tiene lo siguiente

$$
\begin{gathered}
\xi_{2}\left(v ; x_{2}^{*}\right)=0.138-0.062 v_{2}+2.8 \times 10^{-3} /\left(x_{2}^{*}-0.28\right) \\
\xi_{1}\left(v ; x_{2}^{*}\right)=0.0155+0.123 \ln \left(x_{2}^{*}\right)
\end{gathered}
$$

donde $0<x_{2}^{*} \leq x_{2}^{c}=0.28 \mathrm{~g} / \mathrm{L}$. Adicionalmente, consideremos que: (a) la medición de sustrato está contaminada con ruido, el cual fue simulado usando números generados aleatoriamente con una amplitud que corresponde al $10 \%$ de error de medición, e.i., $y=x_{2}\left(1+0.1 \mathrm{~N}_{\mathrm{a}}\right)$, donde $\mathrm{N}_{\mathrm{a}} \in[-1,1]$ representa al número aleatorio generado. (b) Todos los parámetros de la tasa especifica de crecimiento están variando en el tiempo, $\pi_{1}=\pi_{i}^{*}(1.0+\cos (t))$, donde $\pi=$ $\left[\pi_{1}, \pi_{2}, \pi_{3} \pi_{4}\right]=\left[\mu_{\mathrm{M}}, \mathrm{K}_{\mathrm{M}}, \mathrm{Y}_{\mathrm{s}}, \mathrm{k}_{\mathrm{m}}\right]$ es un conjunto de parámetros variantes en tiempo y $\pi^{*}=$ $\left[\pi_{1}^{*}, \pi_{2}^{*}, \pi_{3}^{*}, \pi_{4}^{*}\right]=[0.41,0.01,2.5,0.04]$ es un conjunto de valores nominales de los parámetros contenidos en $\pi$. (c) La concetración de entrada de sustrato fluctúa periódicamente como sigue: $s_{\mathrm{e}}=\mathrm{s}_{\mathrm{e}}^{*}+15 \operatorname{sen}(\mathrm{t})$, donde $\mathrm{s}_{\mathrm{e}}^{*}=200 \mathrm{~g} / \mathrm{L} \cdot \mathrm{Hr}$ es una valor nominal.

En la Figura 18 se muestra la regulación de sustrato en el fermentador de lote alimentado. Los parámetros de control fueron elegidos como sigue: $\mathrm{L}=50, \kappa_{1}=2 \mathrm{y} \kappa_{2}=$ $1(A(\kappa, r)$ tiene sus autovalores localizados en -1$)$ y $K_{1}=-1$. Cabe destacar que: (i) A pesar que $\mathrm{L}$ es muy grande, el ruido no se amplifica. (ii) el orden del controlador es mucho más pequeño, $(\rho+1)=2$, que el orden del sistema, $\mathrm{n}=4$. Evidentemente, la concetración $x_{2}<x_{\mathrm{s}}^{\mathrm{c}}$ $=0.28$ para todo $t \geq 0$ (Fig. 18.a). Aunque para $4<t<13.5 \mathrm{Hr}$ la concentración de etanol, $x_{4}$, crece para $\mathrm{t}=13.5 \mathrm{Hr}$ la concentración de alcohol etílico se mantiene por debajo 0.25 $\mathrm{gr} / \mathrm{L}$. Por ello, el crecimiento de biomasa no se inhibe (ver Figs. 18.c y 18.d). Así el CAR cumple con el objetivo de producción de biomasa por medio de la regulación de concetración sustrato, $\boldsymbol{x}_{2}$, a pesar de (i) errores en modelo, puesto que el controlador no dispone de información acerca de las tasas de crecimiento de biomasa, formación ni consumo de etanol.

(ii) Perturbaciones exógenas, de las cuales el CAR tampoco está provisto, (iii) variaciones 
paramétricas (simulaciones han mostrado que aún cuando las variaciones paramétricas sean más severas que las presentadas en la Figura 18) y, (iv) ruido de medición.
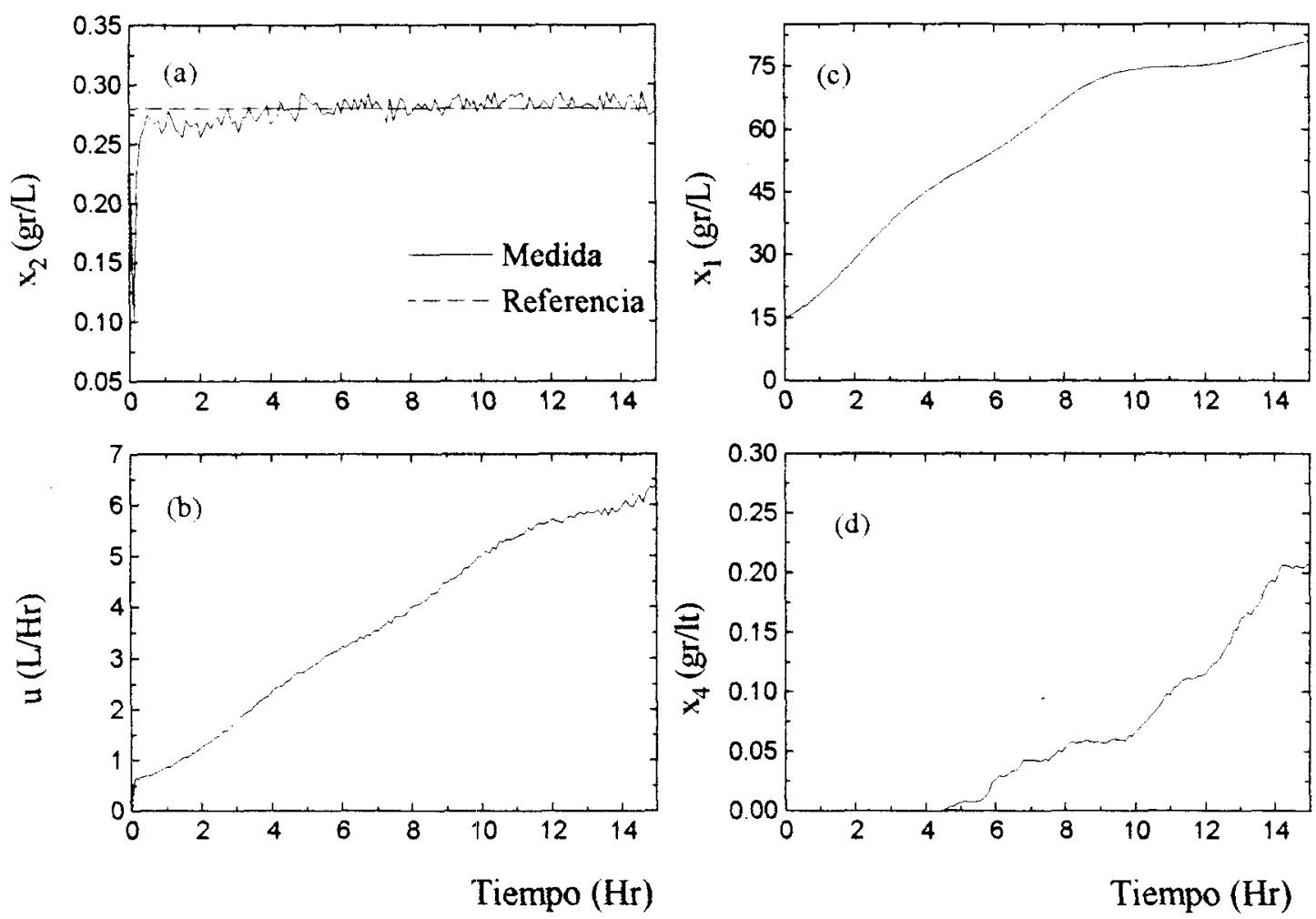

Figura 18. Regulación de sustrato en un fermentador para producción de levadura.

\subsection{Regulación robusta de temperatura en efluente de proceso}

Hasta aquí sólo se ha tratado, por ser el objetivo principal de este trabajo de investigación, con el problema de control en reactores químicos. Sin embargo, frecuentemente los reactores químicos están conectados a otros procesos y es común que la temperatura a la cual se estabiliza el reactor no sea apropiada como efluente. O bien, que dicha temperatura de efluente lleve un exceso de energía, el cual por razones tanto económicas como ambientales, es conveniente recircular para precalentar corrientes de alimentación o servicio. Para esta recuperación energética se utilizan intercambiadores de calor. Así pues, en esta sección se considera el problema de regulación de temperatura en un intercambiador de calor.

El problema de regulación de temperatura mediante intercambiadores es interesante por varias razones. Por un lado, los intercambiadores de calor pertenecen a la clase de sistemas que son totalmente linealizables, es decir, no tienen dinámica interna. La linealización de procesos de intercambio de calor ha sido estudiado en [39] y [40]. En el primero, fueron reportadas algunas reglas de sintonización para el modelo de referencia mientras que, en el segundo, se 
Capitulo cuarto

discute un algoritmo de linealización por retroalimentación para un modelo discretizado de una unidad de intercambio de energía. No obstante, en ninguno de ellos se estudian las propiedades de robustez. Por otra parte, aún cuando el proceso de intercambio de calor es simple, tiene la suficiente estructura para capturar muchas de las características importantes tanto de la linealización por retroalimentación como de compensación de incertidumbres y rechazo (o amortiguamiento) de perturbaciones.

Además, se ha elegido una unidad de intercambio energético para ilustrar algunas propiedades del CAR. Este caso de estudio es útil para conectar las ideas desarrolladas en el $\$ 3$ con las de la generalización discutidas en el presente capítulo. Todo esto, debido a que el modelo de un intercambiador de calor con flujos a contracorriente pertenece a la clase de sistemas totalmente linalizables, cuyo orden es bajo, conservando las características de incertidumbres, tipo de alimentación, requerimientos de desempeño y robustez de los procesos químicos con que se trata en este texto.

En el capítulo anterior se asumió la disposición para retroalimentación de un estimado de la función no lineal incierta que no está asociada a la entrada de control (ver sección 3.1 y sus ejemplos), mientras que, en el presente, las hipótesis no contemplan tal estimado. Pues, precisamente, la conección de ideas consiste en que se dispone de un estimado de dicha función no lineal, el cual puede ser calculado (o se puede determinar por medio de alguna expresión heurística). Particularmente, en esta sección se discutirá un procedimiento para calcular tal estimado a partir de un modelo de referencia obtenido de la identificación del operador de transferencia entrada-salida.

\subsubsection{Modelo dinámico de un intercambiador de calor}

En [39] se presenta un modelo simple, pero realista, para intercambiadores de calor. Para tratar con este problema de control se considerará dicho modelo, donde los flujos de proceso y servicio se ponen en contacto a contracorriente. Luego, sean $x_{1}$ y $x_{2}$ la temperatura de efluente de proceso y la de servicio, respctivamente. $C_{i, t}=C_{i, t}(t)=U(t) / C p_{i}$ un coeficiente de transferencia de calor (donde $U(t)$ es el coeficiente total de transferencia de calor, $C p_{i}$ es la capacidad calorífica $y$, el subindice $i=1,2$, indica efluente de proceso y corriente de servicio, respectivamente). $a$ es el área de intercambio de energía, $M_{p}$ y $M_{c}$ son constantes y $\mathrm{F}_{p}=\mathrm{F}_{p}(\mathrm{t})$ es el flujo másico de corriente de proceso mientras que $u$ es el flujo másico de corriente de servicio. Así pues el modelo dinámico está dado por

$$
\begin{aligned}
& \dot{x}_{1}=\frac{2}{M_{p}}\left[F_{p}\left(x_{1, e}-x_{1}\right)-C_{1, T} a \Delta x(\chi)\right] \\
& \dot{x}_{2}=\frac{2}{M_{c}}\left[C_{2, T} a \Delta x(\chi)+\left(x_{2, e}-x_{2}\right) u\right]
\end{aligned}
$$

donde $\chi=\left(x_{2, \mathrm{e}}, x_{2}, x_{1, \mathrm{e}}, x_{\mathrm{i}}\right) \in \mathbf{R}^{4}$ es un vector de las temperaturas de entrada $\mathrm{y}$ salida del intercambiador. $\Delta x(\chi)$ es el promedio efectivo de diferencia de temperatura. En la práctica, 
se dispone de algunas expresiones heurísticas para calcular el promedio efectivo de diferencia de temperatura, $\Delta x(\chi)$. Estas están dadas por: (i) La media efectiva aritmética $\Delta x(\chi)=\left[\left(x_{1}\right.\right.$ $\left.\left.x_{2 . \mathrm{e}}\right)-\left(x_{1 . \mathrm{e}}-x_{2}\right)\right] / 20$, (ii) por la media efectiva logaritmica

$$
\Delta x(\chi)=\frac{\left(x_{2}-x_{1, e}\right)+\left(x_{2, e}-x_{1}\right)}{\log \left[\frac{x_{2}-x_{1, e}}{x_{2, e}-x_{1}}\right]}
$$

Empíricamente, es sabido que la media logarítmica (65) reproduce mejor el comportamiento del intercambio energético a contracorriente. Es evidente que el modelo puede ser reescrito como el sistema (34).

El objetivo de control es regular la temperatura del efluente, $x_{1}$, en una referencia deseada, $x_{1}^{*}$. Entonces sea $y=x_{1}-x_{1}^{*}$, la salida (medición) del intercambiador. Otra obviedad es que el grado relativo del sistema que modela al proceso es $\rho=\mathrm{n}=2$. Así pues, definiendo el siguiente cambio de coordenadas: $z_{1}=y$ y $z_{2}=2\left[\mathrm{~F}_{\mathrm{p}}\left(x_{2}-x_{1, \mathrm{e}}\right)-\mathrm{C}_{1, \mathrm{~T}} a \Delta x(\chi)\right] / \mathrm{M}_{\mathrm{p}}=f_{1}(\mathrm{z}, w)$. El cual es invertible si y solo si $\partial_{2} \Delta x(\chi)=\partial \Delta x(\chi) / \partial x_{2}=\left[\ln \left(a_{1}\right)+1 / a_{1}-1\right] /\left[\ln \left(a_{1}\right)\right]^{2} \neq 0$, donde $a_{1}=\left(x_{2}-x_{1, \mathrm{e}}\right) /\left(x_{1}-x_{2, \mathrm{e}}\right)$ [8]. Si $0<a_{1}<\infty,\left|\hat{c}_{2} \Delta x(\chi)\right|>\sigma / 2$, donde $\sigma$ es un número positivo. Nótese que si $a_{1} \rightarrow 1$, entonces $\left|\partial_{2} \Delta x(\chi)\right| \rightarrow 1 / 2$, es decir, alrededor de $a_{1}=1$, la media efectiva logarítmica se comporta como la media efectiva aritmética.

Pues bien, con el cambio de coordenas anterior, el sistema (64) pueder ser reescrito en la forma canónica (35) como

$$
\begin{aligned}
& \dot{z}_{1}=z_{2} \\
& \dot{z}_{2}=\alpha(z, d)+\gamma(z, d) u \\
& y=z_{1}
\end{aligned}
$$

donde $\alpha(\mathrm{z}, d)$ y $\gamma(\mathrm{z}, d)$ son funciones no lineales inciertas.

\section{5 .2 El diseño del CAR}

La dificultad que constituyen las incertidumbres, en el problema de regulación de temperatura de efluente en una unidad de intercambio energético, puede ser tratada con el diseño de un controlador del tipo asintótico robusto. Para ello, discutamos primero las implicaciones y significado que tienen las hipótesis (H.4.1)-(H.4.4).

La primer suposición es práctica, pues como ya ha sido dicho, la medición de temperatura es barata y rápida. Así que, además de la salida, se puede asumir que $x_{2}, x_{2, \mathrm{e}}$ están disponibles para retroalimentación por mediciones en línea. En lo que respecta a (H.4.2) y (H.4.3), las perturbaciones exógenas $F_{p}$ y $x_{1, e}$ pueden suponerse acotadas $y$ desconocidas (aunque podrían estar disponibles, puesto que existen eficientes dispositivos para la medición 
de flujo en línea), Sólo se conoce su valor nominal, $\mathrm{F}_{\mathrm{p}}^{*}$ y $x_{1, \mathrm{e}^{*}}^{*}$ Además, los coeficientes de transferencia de calor $\mathrm{C}_{\mathrm{i} . \mathrm{T}}$, son dificilmente conocidos. Finalmente, dado que el sistema es totalmente linealizable, el sistema es de fase mínima.

Aquí, con el objetivo de conectar las ideas desarrolladas en $\S 3$, se hará una pequeña modificación al algoritmo desarrollado en el presente capítulo. Considérese que se dispone de un estimado $\alpha(z)$ de la función no lineal incierta $\alpha(z, d)$ (es decir, se dispone de un estimado de la incertidumbre no asociada con la entrada de control). Para la obtención del sistema dinámicamente equivalente, definase los siguiente: Sea $\gamma(z)$ un estimado de $\gamma(z, d)$. Luego, las desviaciones de los estimados con el término incierto están dadas por: $\delta_{1}(z, d)=$ $\alpha(\mathrm{z})-\alpha(\mathrm{z}, d)$ y $\delta_{2}(\mathrm{z}, d)=\gamma(\mathrm{z}, d)-\hat{\gamma}(\mathrm{z})$. Entonces, la función no lineal aglutinante es: $\Theta(\mathrm{z}, d, u)$ $=\delta_{1}(z, d)+\delta_{2}(z, d) u$ y el estado aumentado $\eta=\Theta(z, d)$, con lo que se tiene el siguiente sistema extendido

$$
\begin{aligned}
& \dot{z}_{1}=z_{2} \\
& \dot{z}_{2}=\eta+\hat{\alpha}(z)+\hat{\gamma}(z) u \\
& \dot{\eta}=\Xi(z, \eta, d, u) \\
& y=z_{1}
\end{aligned}
$$

donde $\Xi(z, \eta, d, u)=\left[\partial_{z 1} \delta_{1}(\mathrm{z}, d)+u \partial_{z 1} \delta_{2}(\mathrm{z}, d)\right] z_{2}+\left[\partial_{z 2} \delta_{1}(z, d)+u \partial_{z 2} \delta(z, d)\right][\eta+\hat{\alpha}(\mathrm{z})+\hat{\gamma}(\mathrm{z}) u]$ $+\left[\partial_{d} \delta_{1}(\mathrm{z}, d)+u \partial_{\mathrm{d}} \delta_{2}(\mathrm{z}, d)\right] d+\delta(\mathrm{z}, d) u$. Por los mismos argumentos discutidos anteriormente, la condición $\Psi(\mathrm{z}, \eta, d, u)=\eta-\Theta(\mathrm{z}, d, u)=0$ implica que el sistema extendido (67) es dinámicamente equivalente al sistema (66).

Así mismo, se puede diseñar un estimador de estos para reconstruir la dinámica de los estados $(z, \eta)$. De nuevo, se propone el siguiente estimador de tipo Luenberger

$$
\begin{array}{ll}
\dot{\hat{z}}_{1}=\hat{z}_{2} & +L \kappa_{1}\left(z_{1}-\hat{z}_{1}\right) \\
\dot{z}_{2}=\hat{\eta}+\hat{\alpha}(\hat{z})+\hat{\gamma}(\hat{z}) u & +L^{2} \kappa_{2}\left(z_{1}-\hat{z}_{1}\right) \\
\dot{\hat{\eta}}= &
\end{array}
$$

donde las constatntes $\kappa_{i}^{\prime} s$ son elegidas tales que el polinomio $P_{3}(s)=s^{3}+\kappa_{3} s^{2}+\kappa_{2} s+\kappa_{1}=$ 0 es Hurwitz y $\mathrm{L}_{>}>\mathrm{L}^{*}>0$ es un parámetro de ajuste. Así que la ley de control viene a ser

$$
u=\frac{1}{\hat{\gamma}(\hat{z})}\left[-\hat{\eta}-\hat{\alpha}(\hat{z})-K_{2} \hat{z}_{2}-K_{1} \hat{z}_{1}\right]
$$

y una vez más, para disminuir el efecto en la salida del fenómeno de sobredisparo, la ley de control se puede modificar mediante una función de saturación para obtener $u^{\mathrm{s}}=S a t\{u\}$, donde $\operatorname{Sat}\{\boldsymbol{u}\}: \mathbf{R} \rightarrow \mathscr{B} \subset \mathbf{R}$, donde $\mathscr{B}$ es un conjunto acotado es una función de saturación tal 
que: Si $u \geq u^{\text {sup }}, u^{\text {s }}=u^{\text {sup }}$. Si $u \leq u^{\text {inf }}, u^{\text {s }}=u^{\text {inf }}$ y si $u^{\text {int }}<u<u^{\text {sup }}, u^{\text {s }}=u$. Las propiedades de estabilización del controlador anterior ya han sido discutidas, y sería redundante mencionar algo al respecto (además de correr el riesgo de perder de vista el objetivo de esta sección).

Ahora bien, el CAR (68),(69) requiere de expresiones de los estimados $\dot{\alpha}(z)$ y $\hat{\gamma}(z)$. Si $x_{2}$ y $x_{z, e}$ están disponibles para retroalimentación por mediciones en línea, $\hat{\gamma}(z)=$ $4 C_{2.1}^{*} a^{*}\left(x_{2, \mathrm{e}}-x_{2}\right) \partial_{z 2} \Delta x(\chi)$. La obtención de la derivada parcial $\partial_{\mathrm{z} 2} \Delta x(\chi)$ puede no ser una tarea fácil. Para el caso de la aproximación del promedio efectivo de diferencias de temperatura por la media aritmética, resulta que $\hat{\gamma}(\mathrm{z})=-2 \mathrm{C}_{2, \mathrm{~T}}^{*} a^{*}\left(x_{2, \mathrm{e}}-x_{2}\right) / \mathrm{M}_{\mathrm{c}}$. Sin embargo, es conveniente establecer un procedimiento metodológico para el cálculo de los estimados de las funciones inciertas. En la siguiente subsección se propone un método sistemático para la obtención de dichos estimados a partir de la identificación del operador de transferencia entrada-salida del proceso.

\subsubsection{Cálculo de los estimados requeridos para retroalimentación}

Como fue discutido en la sección 3.3, el diseño del CAR puede estar basado en un modelo de referencia lineal. En una aproximación lineal, los estimados $\hat{\alpha}(z)$ y $\hat{\gamma}(z)$ son calculados a partir de experimentos a lazo abierto en el equipo (con sus condiciones de operacion actuales), o bien, de una linealización alrededor de un punto de operación nominal.

Del sistema (66), se puede representar como una ecuación diferencial de segundo orden en $z_{1}$ como sigue: $\ddot{z}_{1}-\alpha\left(z_{1}, \dot{z}_{1}, d\right)=\gamma\left(z, \dot{z}_{1}, d\right) u$. Si suponemos que ésta se comporta linealmente, entonces el operador de transferencia entrada-salida de la planta es $G(s)=K_{p} /$ $\left(\mathrm{s}^{2}+a_{1} \mathrm{~s}+a_{2}\right)$. Cabe puntualizar que la función de transferencia $\mathrm{G}(\mathrm{s})$ tiene sus polos en el hemiplano izquierdo complejo. De las simulaciones presentadas en [39] se puede observar que el intercambiador de calor (64) presenta respuestas sobreamortiguadas para la mayoría de las condiciones de operación. Consecuentemente, uno puede elegir el siguiente operador de transferencia

$$
\frac{Y(s)}{U(s)}=G(s)=\frac{K_{p}}{\left(\tau_{1} s+1\right)\left(\tau_{z} s+1\right)}
$$

donde $\tau_{1}, \tau_{2}>0$ son los tiempos característicos del proceso. Si se identifica a $Y(s)$ y U(s) como $z_{1}=x_{1}-x_{1}^{*}$ y $u-u^{*}$, respectivamente, entonces la planta (70) puede ser realizada en espacio de estados como

$$
\begin{aligned}
& \dot{z}_{1}=z_{2} \\
& \dot{z}_{2}=\frac{-\left[z_{1}+\left(\tau_{1}+\tau_{2}\right) z_{2}+K_{p}\left(u-u^{*}\right)\right]}{\tau_{1} \tau_{2}}
\end{aligned}
$$

de la comparación directa entre los sistemas (71) y (66) se obtienen las expresiones para los estimados. Entonces $\hat{\alpha}(z)=-\left[z_{1}+\left(\tau_{1}+\tau_{2}\right) z_{2}\right] / \tau_{1} \tau_{2}$ y $\gamma(z)=K_{p} / \tau_{1} \tau_{2}$. De acuerdo con el análisis de estabilidad del sistema a lazo cerrado (ver la sección 4.2), $|\gamma(z)|$ debe ser una cota superior 
Capitulo cuarto

de $|\gamma(z, d)|$ en una cierta región de interés. Por consiguiente, en la etapa de identificación de los parámetros $K_{p}, \tau_{1}$ y $\tau_{2}$, la magnitud de la ganancia estática, $K_{p}$, debe ser sobreestimada mientras que la magnitud de los tiempos característicos, $\tau_{1}$ y $\tau_{2}$, debe ser subestimada. Esto es, el modelo (70) debe ser construido en base a la dinámica más rápida del proceso.

Para ilustrar la estimación de los parámetros de la planta (70), se han considerado los valores nominales del modelo que fueron reportados en [39]. La identificación fue realizada con el siguiente procedimiento. Primero, se indujo en la entrada del proceso un cambio en escalón de $+0.25 \%$. Luego, los parámetros fueron ajustados a la respuesta dinámica de la salida $y$. Así, los valores aproximados de los parámetros son: $\mathrm{K}_{\mathrm{p}}=-0.2{ }^{\circ} \mathrm{F} \mathrm{min} \mathrm{m}^{-1} \mathrm{lbm}^{-1}, \tau_{1}$ $=0.4 \min y \tau_{2}=0.3 \mathrm{~min}$. Por lo tanto, $\alpha(z)=-8.3 z_{1}-5.8 z_{2}$ y $\gamma(z) \approx 1.9$.

Las ganancias de control $\mathrm{K}_{1}$ y $\mathrm{K}_{2}$ fueron elegidas tales que los tiempos característicos del sistema ideal a lazo cerrado $\ddot{z}_{1}+K_{2} \dot{z}_{1}+K_{1} z_{1}=0$ son $\tau_{c, 1}=\tau_{c, 2}=0.25$ min (es decir, las raíces del polinomio característico $s^{2}+K_{2} s+K_{1}=0$ son impuestas en -4 ). $O$ sea que $K_{2}=$ $16^{\circ} \mathrm{Fmin}^{-1} \mathrm{lbm}^{-1}$ y $_{1}=8{ }^{\circ} \mathrm{Fmin}^{-1} \mathrm{lbm}^{-1}$. Al igual que en la aproximación por modelo interno, la idea de esta sintonización es inducir una respuesta a lazo cerrado ligeramente más rápida que a lazo abierto. De esta manera, un mayor esfuerzo de control debe ser aplicado para amortiguar perturbaciones y seguir cambios de puntos de referencia.

\subsection{Simulaciones numéricas}

Para evaluar la capacidad del CAR para rechazar perturbaciones, se consideró que $x_{1}$ $=x_{1}^{*}+5 \operatorname{sen}(0.5 \mathrm{t})$. La temperatura de efluente deseada es $x_{2}^{*}=130$ oF. A $\mathrm{t}=20$ min se indujo un cambio en escalón (de $25 \mathrm{lbm} / \mathrm{min}$ a $30 \mathrm{lbm} / \mathrm{min}$ ) en el flujo del proceso $\mathrm{F}_{\mathrm{p}}$. Las constantes de estimación fueron elegidas tales que la matriz compañera del error de estimación $A(\kappa, r)$ tiene sus valores propios localizados en -1. El desempeño del CAR se muestra en la Figura 19. El valor del parámetro de ajuste es $\mathrm{L}=5$, así que la dinámica del estimador (68 es 20 veces más rápida que la dinámica nominal de sistema controlado.

Nótese que la temperatura de efluente es llevada prácticamente a la referencia. Las oscilaciones en las acciones de control son debidas al amortiguamiento de perturbaciones requerido. Es decir, el comando de control absorbe las fluctuaciones de la temperatura alimentado al intercambiador. De hecho, el error de regulación no esmayor que $0.2{ }^{\circ} \mathrm{F}$. Cabe mencionar que para un mayor flujo másico $F_{p}$, el sistema a lazo cerrado es más sensible a las perturbaciones.

Ahora considérese un cambio de $+20 \%$ en el coeficiente de transferencia de calor. El cambio fue inducido a $\mathrm{t}=20 \mathrm{~min}$. La entrada de control, entonces se ajusta a las nuevas condiciones de remoción energética. En la Figura 20 se presenta el desempeño del CAR. 

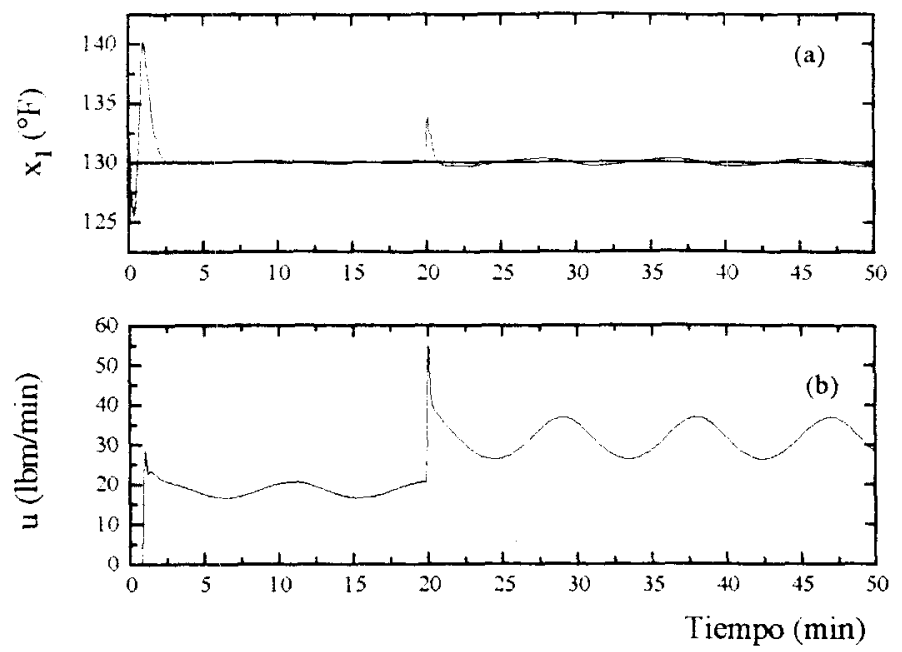

Figura 19. Desempeño del CAR en la regulación de temperatura de efluente ante cambio en tlujo de proceso.

Una vez más, las oscilaciones estacionarias que depliega el control son debidas al amortiguamiento de las perturbaciones exógenas. Es importante detacar que ante las perturbaciones simuladas, la salida del proceso es regulada sin presentar sobredisparos.muy severos. Empero, las acciones de control si presentan un pico importante [8].
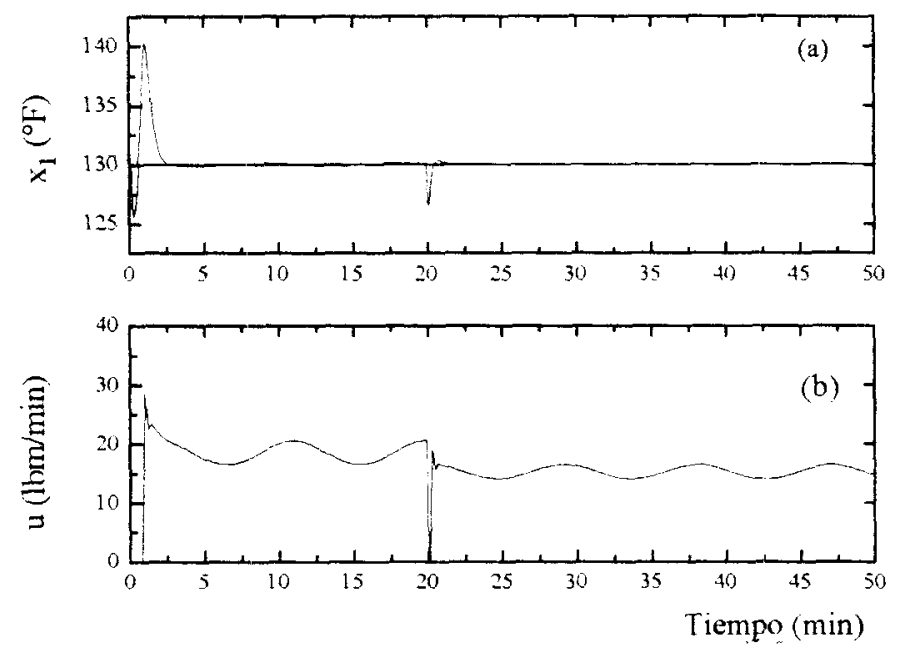

Figura 20. Regulación de temperatura de efluente ante cambio en coeficiente de transferencia de calor. 
Capitulo cuarto

\subsection{Comentarios sobre la linealización asintótica robusta}

En este capítulo se desarrolló un algoritmo de diseño para la linealización asintótica robusta por retroalimentación de mediciones. Seguiendo las ideas discutidas anteriormente, el controlador resultante del algoritmo propuesto consta de dos partes: (a) Un Compensador dinámico de incertidumbres y (b) una ley de control de tipo linealizante. El compensador dinámico está constituido por un obsrevador de estados. En particular, se ha utilizado un observador de tipo Luenberger. La aproximación de la ley de control sólo usa valores estimados de las incertidumbres y de los estados no medidos (los cuales son provistos por el observador).

Para ello, se construye un sistema dinámico equivalente al sistema original. El sistema dinámicamente equivalente es un sistema en espacio extendido en un orden de magnitud. Una condición de invarianza (restricción algebraica) garantiza que las soluciones del sistema original y el extendido sean iguales. Así, la solución del sistema original es una proyección del sistema extendido. Luego, una ley de control que estabiliza al sistema extendido provee la estabilidad a lazo cerrado en la planta.

Dado que los valores estimados son obtenidos de un observador de alta ganancia, se deben esperar sobredisparos. Para disminuir el efecto de las señales de pico en la salida, la ley de control puede ser modificada mediante una función de saturación cuyo objetivo es restringir las acciones de control a un conjunto acotado. Por otra parte, se requiere un único parámetro de estimación (ganancia de alta frecuencia, L). El cual es sintonizado por imposición de polos. Sin embargo, este procedimiento no es apropiado en presencia de mediciones ruidosas. Un método óptimo de sintonización (por ejemplo, usado en filtraje óptimo de Kalman) puede ser usado. Más aún, se puede sugerir un estimador de estados distinto, como un observador basado en modo deslizante o una esquema de redes neuronales, etc. Por supuesto que una etapa de filtrado posterior a la de medición es una alternativa en aplicaciones prácticas.

Luego, el control asintótico robusto fue implementado (por medio de simulaciones numéricas) a diferentes procesos. (i) Un reactor de lecho fluidizado permite ejemplificar el diseño del CAR en reactores no isotérmicos con dinámica de chaqueta. Aquí se ilustró la disminución del efecto que ofrece la función de saturación. Aunque la acciones de control se saturan unos cuantos segndos ante cambios de referencia, éstas no se degradan (es decir, no se presenta el fenómeno de $w$ ind $u p$ ). Las perturbaciones son rechazadas sin grandes esfuerzos de control. (ii) Después, el CAR fue usado para regular sustrato en un fermentador de lote alimentado. Es este caso, el orden del estimador de estados es mucho menor que el del sistema a controlar. A pesar que variaciones paramétricas fueron simuladas, la concentración de sustrato se mantiene en una vecindad muy cercana a la referencia. (iii) También el esquema de control propuesto fue implementado para regular la temperatura de efluente en una unidad de intercambio de calor donde los flujos se alimentan a contracorriente. Este ejemplo fue un pretexto para presentar un procedimiento de cálculo de los estimados de las funciones inciertas (tanto de la asociada a la entrada de control como la que no lo está) a partir de la identificación práctica (o bien de expresiones heurísticas). 
Linealización asintótica robusta de procesos quimicos

Finalmente, se puede concluir que se ha desarrollado un algoritmo de control basado en geometría diferencial con propiedades de robustez ante: errores en modelo, variaciones paramétricas, perturbaciones exógenas y endógenas. Además con ciertas características de filtrado de mediciones ruidosas (bajo condiciones restrictivas). Adicionalmente, el objetivo de desempeño se cumple aceptablemente. La estabilización (o regulación) es alcanzada en una forma práctica sin excesivos esfuerzos en las acciones de control.

Empero, desde el punto de vista de teoría de control es conveniente relajar la hipótesis que asegura que el grado relativo de la planta está bien definido para todo tiempo. Además, aún cuando la elección de un obsevador de tipo Luenberger fue muy conveniente para analizar la estructura del algoritmo propuesto, se deben explorar otras alternativas de estimación. Tal vez un observador de estados basado en modo deslizante sea apropiado, pues se conservaría la estructura del CAR pero con la disminución del efecto de mediciones ruidosas. $O$ bien, un filtro óptimo de Kalman para contar con un método sistemático de sintonización. Quizá un esquema de estimación basado en redes neuronales. Otra opción es la combinación de diferentes esquemas. Adicionalmente, en el análisis de robustez no se ha contemplado la presencia de dinámicas no modeladas.

Todas estas congeturas y casos van más allá de los objetivos del presente trabajo. No obstante, en el siguiente capítulo se presentan algunos ejemplos para ilustrar las líneas a seguir en trabajos futuros. El estudio de estos ejemplos no ha sido concluído. Aún no se cuenta con un algoritmo sistemático para el diseño de un controlador robusto. Tampoco se ha analizado su desempeño ni examinado su estabilidad a lazo cerrado. De hecho, se presentan como resultados preliminares con el objetivo de vislumbrar la relajación de algunas hipótesis y ampliar la metodología.

Los casos tratados son: (i) La estabilización robusta de sistemas dinámicos con grado relativo no bien definido. Para éste, se toma un ejemplo sencillo, pero muy ilustativo, sin significado en procesos químicos. Se aborda el problema de seguimiento. (ii) La estabilización de procesos químicos con dinámica no modelada. Se considera una unidad de intercambio energético donde se ignora la dinámica el actuador. (iii) Se realiza un modificación a la ley de control (44) para mejorar el desempeño de las acciones de control en la presencia de mediciones ruidosas. La modificación es una variación de la retroalimentación (41) dada por la dependencia de ésta con una superficie deslizante. Se recurre a un reactor exotérmico, donde la reacción $\mathrm{A} \rightarrow \mathrm{B}$ se lleva a cabo, para hacer una comparación entre la ley de control (44) y la modificación hecha. 


\section{Algunas Extensiones a los Resultados Obtenidos}


Algunas Extensiones a los Resultados Obtenidos

Los resultados presentados en este capítulo son preliminares. La formalización de éstos se encuentra en desarrollo. Una vez concluidos se someterán a revisión para su posible publicación. 
Capitulo Quinto

\section{Algunas extensiones a los resultados obtenidos}

Los ejemplos discutidos en este capítulo son presentados como problemas a tratar en trabajos futuros, más que como resultados formales. Aquí se esbozan tanto la formulación del problema como el procedimiento a seguir para cada uno de los casos.

\subsection{Seguimiento robusto en una clase de sistema dinámico}

Considérese el sistema dinámico dado por

$$
\begin{aligned}
& \dot{x}_{1}=x_{2}-x_{1} u \\
& \dot{x}_{2}=u \\
& y=x_{1}
\end{aligned}
$$

donde $y=x_{1}$ es la salida. Supóngase que la señal de referencia está dada por $y_{\mathrm{r}}=a_{\mathrm{r}} \operatorname{sen}\left(\omega_{\mathrm{r}} \mathrm{t}\right)$, $a_{\mathrm{r}}$ es la amplitud y $\omega_{\mathrm{r}}$ es la frecuencia de la señal de referencia, respectivamente.

El problema de seguimiento consiste en diseñar una ley de control $\boldsymbol{u}=\boldsymbol{u ( t )}$ tal que la salida $y$ siga la señal referencia $y_{\mathrm{r}}$. Conforme a la metodología de linealización por retroalimentación basada en geometía diferencial [1]. Tomése la derivada de la salida, $y=x_{1}$, con lo cual se tiene que $y=x_{2}-x_{1} u$, es obvio que el grado relativo del sistema (72) es $\rho=$ 1. Luego, definiendo un cambio de coordenadas $z_{1}=y$, Se puede obtener el siguiente sistema dinámico $\dot{z}_{1}=x_{2}-z_{1} u$. De la cual se puede diseñar una retroalimentación de estados dada por $u=\left[x_{2}+\mathrm{K}_{1}\left(y-y_{\mathrm{r}}\right)\right] / \mathrm{z}_{1}, \mathrm{z}_{1}=a_{\mathrm{r}} \operatorname{sen}\left(\omega_{\mathrm{r}} \mathrm{t}\right)$. Evidentemente, el grado relativo no está bien definido para todo $z_{1}$. Derivando de nuevo la salida, se tiene que $\ddot{y}=\left(1-x_{2}\right) u+x_{1} u^{2}-x_{1} \dot{u}$. Con esto último se llega al siguiente sistema dinámico

$$
\begin{aligned}
& \dot{z}_{1}=z_{2} \\
& \dot{z}_{2}=\alpha(z, \dot{u})+\gamma(z) u \\
& y=z_{1}
\end{aligned}
$$

donde $\alpha(z, u)=-z_{1} \dot{u}$ y $\gamma(z)=1-z_{2}$. Claro está que una retroalimetación dada por la siguiente expersión $u=\left[-\alpha(z, \dot{u})+\mathrm{K}_{1}\left(y-y_{\mathrm{r}}\right)+\mathbf{K}_{2}\left(\dot{y}-\dot{y}_{\mathrm{r}}\right)\right] / \gamma(z)$ es una ley de control no causal.

Ahora bien, considérense las siguientes suposiciones: (S.I) Se dispone de un valor estimado, $\gamma(z)$, de $\gamma(z)$ estrictamente diferente de cero. $(S .2) \gamma(z)$ está acotada. $(S .3) \alpha(z, i)$ no está disponible para retroalimentación. Cabe decir que las hipótesis anteriores son congruentes con aquellas elaboradas a lo largo del texto.

Definiendo $\delta(z)=\gamma(z)-\gamma(z), \Theta(z, u, u)=\alpha(z, u)+\delta(z) u$ y $\eta=\Theta(z, u, \dot{u})$. Se puede 
reescribir al sistema (73) es espacio extendido como

$$
\begin{aligned}
& \dot{z}_{1}=z_{2} \\
& \dot{z}_{2}=\eta+\dot{\gamma}(z) u \\
& \dot{\eta}=\Xi(z, u, \dot{u}, \dot{u}) \\
& y=z_{1}
\end{aligned}
$$

donde $\Xi(z, u, \dot{u}, \ddot{u})=-z_{2} \dot{u}-z_{1} \ddot{u}-\eta-\hat{\gamma}(z) u$. Entonces, se puede diseñar un estimador de estados. Nuevamente, se propone el siguiente obsevador de tipo Luenberger

$$
\begin{aligned}
& \dot{z}_{1}=\hat{z}_{2} \quad+L k_{1}\left(z_{1}-\hat{z}_{1}\right) \\
& \dot{\hat{z}}_{2}=\hat{\eta}+\hat{\gamma}(\hat{z}) u+L^{2} x_{2}\left(z_{1}-\hat{z}_{1}\right) \\
& \dot{\hat{\eta}}=\quad+L^{3} k_{3}\left(z_{1}-\hat{z}_{1}\right)
\end{aligned}
$$

y la aproximación a la ley de control linealizante está dada por

$$
u=\frac{1}{\hat{\gamma}(\hat{z})}\left[-\hat{\eta}+K_{1}\left(y-y_{r}\right)+K_{2}\left(\dot{y}-\dot{y}_{r}\right)\right]
$$

Se puede usar una función de saturación para disminuir el efecto de sobredisparos en la salida controlada. Sin embargo, se utilizará la ley de control (76) para presentar, por medio de simulaciones, el comportamiento del sistema a lazo cerrado.
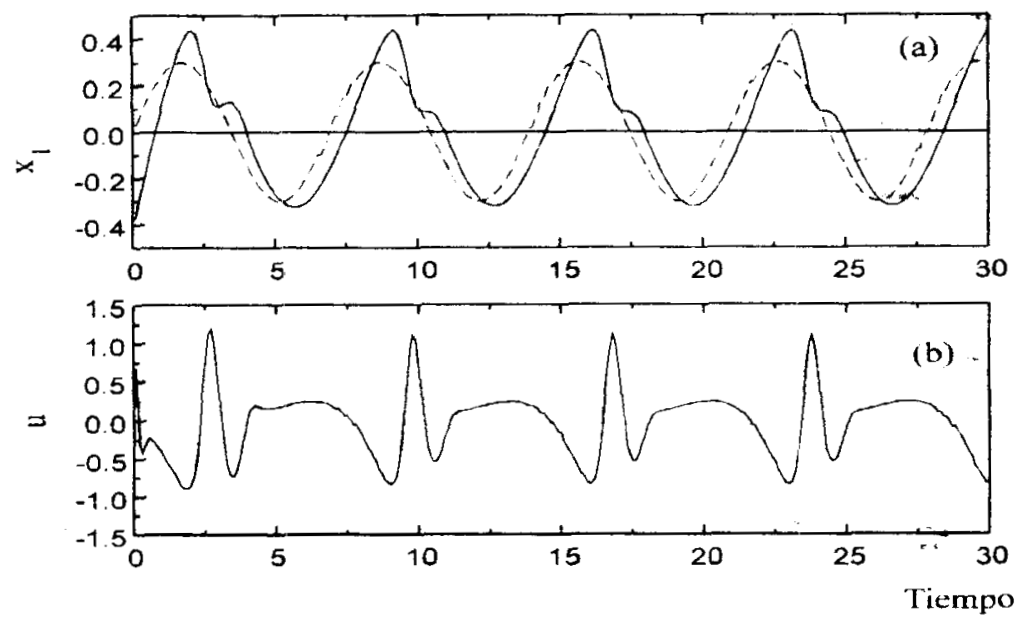

Figura 21. Seguimiento de una señal periódica. $\cdots y_{1}, \ldots y=x_{i}$. 
Capitulo Quinto

Sea $z_{2}^{*}$ un valor nominal de $z_{2}$ constante tal que $\gamma(z)$ sea estrictamente diferente de cero, lo cual implica que $\left|z_{2}^{*}\right| \neq 1$. En la figura 21 se muestra el seguimiento de la señal de referencia. Los valores de los parámetros de control $\mathrm{K}_{1}$ y $\mathrm{K}_{2}$ fueron elegidos de tal manera que el polinomio $s^{2}+K_{2} s+K_{1}=0$ tiene sus raíces en -1 . Asi mismo, las constantes de estimación $\kappa_{1}, \kappa_{2} y \kappa_{3}$ fueron elegidas tales que $s^{3}+\kappa_{3} s^{2}+\kappa_{2} s+\kappa_{1}=0$ es Hurwitz. El valor nominal $z_{2}^{*}=0.0$ y el valor de parámetro de alta frecuencia foe elegido como $L=5$

En esta sección se ha esbozado un procedimiento para el problema de seguimiento. El costo a pagar por lograr el seguimiento cuando el grado relativo no está definido para todo $x_{0} \in \mathbf{R}^{n}$, es que el orden del compensador dinámico crece. Adicionalmente, se imponen restricciones a las acciones de control (debidas a la no causalidad de la ley de control). En particular, con el controlador (75),(76) no se pueden seguir señales con amplitud arbitraria y la región de atracción del sistema a lazo cerrado puede modificarse a diferentes valores de la amplitud y frecuencia de la referencia. Obviamente, estas y otras dificultades no se han estudiado completamente. Los resultados que se obtengan serán reportados a la brevedad.

\subsection{Control robusto ante dinámicas no modeladas}

En los capítulos anteriores no se trató con el problema de control en presencia de dinámica no modelada. Aquí se presenta un ejemplo con el fin de ilustrar la capacidad del CAR para estabilizar sistemas donde se desconoce la diámica del actuador. Por medio de simulaciones numéricas, se muestra que el controlador asintótico robusto puede ofrecer estabilidad en un sitema a lazo cerrado a pesar de que la dinámica del actuador no esté modelada. Aunque el estudio teórico (incluyendo el análisis de estabilidad, la construcción de un algoritmo sistemático de diseño y la metodología de implementación/sintonización) de este problema está en desarrollo, se han llevado a cabo algunas simulaciones para ilustrar los resultados preliminares obtenidos.

Se dice que un sistema tiene dinámica no modelada cuando parte del proceso posee dinámica más rápida que el proceso mismo y ésta no es tomada en cuenta en el modelo. Existen algunos casos típicos: (a) El modelo dinámico del actuador no es tomado en cuenta en el diseño de la ley de control. Por ejemplo, pensemos en válvulas de control de flujo conectadas a procesos químicos. El tiempo característico de éstas es del orden de minutos (rara vez de horas) mientras que el de un proceso es de horas, tal vez de días ${ }^{4}$. Ejemplos de ello son las columnas usadas en la industria petrolera, reactores de polimerización o biológicos. (b) Parte del modelo dinámico del proceso no es considerado. Este es el caso de sistemas reactantes donde algún compuesto intermediario tiene un estado de transición. La clase de reacción con compuesto intermediario es muy común en reacciones orgánicas y se caracteriza por tener cinética de reacción mucho más rápida que la de el(los) reactante(s) y producto(s) final(es) y, por ello, frecuentemente tal compuesto no es medible. Con el fin de ser explícito, considérese que procede lo siguiente $A \rightarrow B \rightarrow C$. Supóngase que la tasa de

\footnotetext{
${ }^{4}$ Sólo algunos casos muy especializados poseen un tiempo característico de menor orden de magnitud, como los reactores de lecho fluidizado, secadores, columnas de destilación en industria farmaceutica, en cuyo caso se requiere una respuesta de actuador más rápida.
} 
conversión de $\mathrm{B} \rightarrow \mathrm{C}$ es más rápida que la de $\mathrm{A} \rightarrow \mathrm{B}$ de tal manera que la cinética de reacción pueda ser modelada tomando (únicamente) $A \rightarrow C$. Estríctamente, un reactor con el primer esquema de reacción presentará comportamiento dinámico distinto a uno con el último esquema [2]. Sin embargo, para fines de producción y si el compuesto intermediario no es aislable, frecuentemente se desprecia la dinámica asociada al producto intermediario.

Aquí, nada más se considera el caso donde se ignora la dinámica del actuador. En particular, pensemos en una válvula de control de flujo conectada a un intercambiador de calor. Considérese la unidad de intercambio energético discutida en la sección 4.5. Asúmase que la válvula esta gobernada por la siguiente dinámica de primer orden

$$
\mathscr{F}=\frac{u}{\tau_{a} s+1}
$$

donde $\mathscr{F}$ es la tasa de lujo de fluido actual, $\tau_{a}$ es el tiempo característico del actuador y $u$ es el comando de control calculado (flujo de corriente de servicio, ver sección 4.5). Nótese que si $\tau_{a}=0$ (respuesta de actuador infinitamente rápida), entonces $\mathscr{F}=u$, en cuyo caso la estabilidad a lazo cerrado sigue los resultados presentados en el capítulo inmediato anterior.
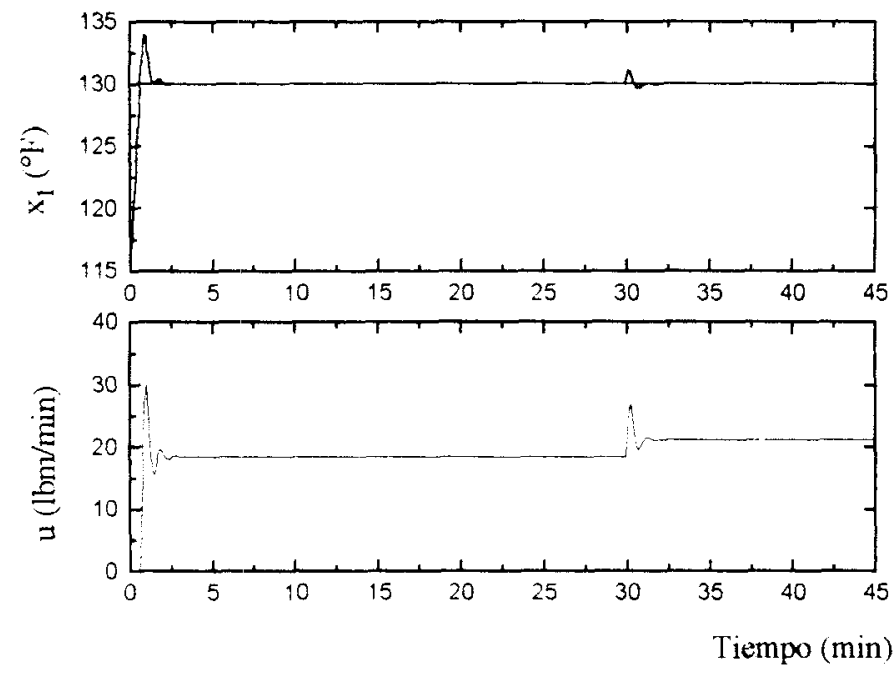

Figura 22. Regulación de temperatura en un intercambiador de calor en presencia de dinámica no modelada

Para realizar las simulaciones, se eligió a $\tau_{a}=0.1 \mathrm{~min}$ y los mismos valores usados en la implementado hecha en sección 4.5 (tanto para los parámetros de control como para los de estimación). Además, se asumió que $x_{1}=x_{1}^{*}$ (es decir, no hay perturbaciones). En la Figura 22 se presenta el desempeño del controlador para un cambio en escalón en la temperartura de servicio, $x_{2, \mathrm{e}}\left(\right.$ de 70 a $75{ }^{\circ} \mathrm{F}$ en $\mathrm{t}=30 \mathrm{~min}$ ). Debido al retardo inducido por el actuador, el 
Capituio Quinto

sistema a lazo cerrado es ligeramente más lento que en el caso sin su dinámica. Sin embargo, el desempeño de la retroalimentación es muy bueno (Fig. 7.a). Contrariamente al caso simulado en la sección 4.5, casi no presenta sobredisparo. Este comportamiento es ocasionado por la dinámica de la válvula que actua como un suavizador de las acciones de control (tal y como un filtro pasabajos, [23]). En la Figura 7.b se muestra la tasa de flujo de la corriente de servicio inducida por el CAR. También, por el retardo inducido por la dinámica (77), el comportamiento de $u$ exhibe oscilaciones (desplegadas antes de alcanzar el estado estacionario).

Por supuesto que se requiere tratar con el problema de control de sistemas reactantes con dinámica no modelada en una manera más sistemática. Además, se deben analizar las propiedades de robustez y desempeño. Sin embargo, el caso discutido aquí permite vislumbrar la viabilidad del CAR para estabilizar (o en su caso regular) procesos químicos a pesar de dinámica no modelada.

\subsection{Una modificación para el CAR}

A lo largo del texto se ha propuesto un esquema de estimación de estados basada en observadores de tipo Luenberger. Esto ha sido provecho para analizar la estructura del controlador y compararlo en terminos clásicos. Sin embargo, dado que el estimador de estados considerado es un observador de alta ganacia, se pueden presentar sobredisparos y amplificación de ruido. El primer fenómeno es provocado por el ímpetu en la estimación de incertidumbres mientras que el segundo es consecuencia de que el ruido en la medición afecta directamente al estimado del estado aumentado con una amplificación del orden de $\mathrm{L}^{\rho-1} \mathrm{y}$, para valores grandes de la ganacia alta frecuencia, $\eta$ puede distar de $\eta$ (ver el sistema (43)). Así, en la presencia de ruido, y para grandes valores de L, es posible que no se logre el objetivo de control (o peor aún, el sistema a lazo cerrado sea inestable).

Dado que, de acuerdo con la reflexión anterior, el estimador (43) puede inducir acciones de control no deseadas. Se deben estudiar modificaciones alternativas, además de las discutidas otros capítulos, para el diseño de la ley de control (44). Esta es otra línea de investigación que actualmente está en desarrollo y aquí sólo se esboza un algoritmo de diseño para obtener una retroaliemtación robusta aún presencia de mediciones ruidosas.

Considérese el sistema no lineal (40), el cual puede ser controlado mediante la ley de control estabilizante (41). La ley de control por retroalimentación (41) está dada explícitamente por $u(z)=\left[-\eta+K^{\mathrm{T}} z\right] / \gamma(z)$ y garantiza que el sistema a lazo cerrado es estable (Proposición 4.1). Ahora suponga que una función auxiliar dependiente de la entrada [41] $\mathrm{s}(\mathrm{z}, u)=u(\mathrm{z})+\left[-\eta+\mathrm{K}^{\mathrm{T}} \mathrm{z}\right] / \hat{\gamma}(\mathrm{z})$ es sintetizada y propuesta como una candidata a superficie deslizante sobre la cual la siguiente dinámica es impuesta: $\dot{s}(z, u)=-g_{d} \operatorname{sgn}\{s(z, u)\}$, con ganancia constante, $\mathrm{g}_{\mathrm{d}}$, estrictamente positiva $\mathrm{y}$ suficientemente grande. Nótese que las trayectorias de la dinámica impuesta llegan a $s(x, u)=0$, en tiempo finito $\mathrm{t}^{*}$ dado por $\mathrm{t}^{*}=$ $|s(z(0), u(0))| / g_{d}$. Una vez alcanzada la condición anterior se genera un movimiento deslizante sobre una variedad dependiente de la entrada [41]. Luego de algunas sencillas manipulaciones se obtiene la siguiente dinámica del controlador discontinuo de modo deslizante 


$$
\dot{u}=-\left\{\frac{\partial}{\partial z}\left(\frac{-\eta+K^{T} z}{\hat{\gamma}(z)}\right)\right\} \alpha(z, u)-g_{d} \operatorname{sgn}\left\{u+\frac{-\eta+K^{T} z}{\hat{\gamma}(z)}\right\}
$$

la cual, obviamente, representa una ecuación diferencial no lineal variante en tiempo con lado derecho discontinuo.

Luego de integrar (78) y un simple reacomodo de variables se tiene que

$$
s(z(t), u(t))=-s(z(0), u(0))-g_{d} \int_{0}^{t}\left\{s g r\left(u(\sigma)+\frac{-\eta(\sigma)+K^{T} z(\sigma)}{\hat{\gamma}(z(\sigma))}\right)\right\} d \sigma
$$

de donde es directo probar que, para cualquier valor inicial de $s(z, u)$, la condición $s\left(z\left(t^{*}\right), u\left(t^{*}\right)\right)$ $=0$ es alcanzada en el tiempo finito $t^{*}$.

Finalmente la ley de control es reescrita en coordenadas $(\hat{z}, \hat{\eta})$ como sigue

$$
\begin{aligned}
& u=\tilde{u}+\frac{\hat{\eta}-K^{T_{\hat{z}}}}{\hat{\gamma}(\hat{z})} \\
& \tilde{u}=-g_{d} \operatorname{sgn}\left(u+\frac{-\hat{\eta}+K^{T_{\hat{z}}}}{\hat{\gamma}(\hat{z})}\right)
\end{aligned}
$$

donde $(\hat{z}, \eta)$ son los valores estimados (obtenidos mediante el observador $(43))$ de $(z, \eta)$. Nótese que la ley de control (80) no es modificada por la función de saturación, $\operatorname{Sat}\{\cdot\}$, sino por una dependencia de la entrada de una superficie deslizante [41].

Considérese el problema de estabilización de un RCTA exotérmico donde procede la reacción $\mathrm{A} \rightarrow \mathrm{B}$ con tasa de reacción tipo Arrenhius de primer orden en el punto $x^{*}=\left(x_{1}^{*}, x_{2}^{*}\right)$ $=(0.5,400)$ (ver ejemplo 1.1$)$. El modelo de reactor está dado por

$$
\begin{gathered}
\dot{x}_{1}=\theta\left(x_{1, \mathrm{e}}-x_{1}\right)+R(x) \\
\dot{x}_{2}=\theta\left(x_{2, \mathrm{e}}-x_{2}\right)-\beta(\mathrm{x}) R(x)+\gamma\left(x_{2}-u\right)
\end{gathered}
$$

donde $u \in \mathbb{R}$ es la entrada de control $x_{1} \in \mathbf{R}$ es la concentración de reactante y $x_{2} \in \mathbb{R}$ la temperatura del reactor. Ya ha sido probado que para estabilizar el RCTA modelado por el sistema anterior, es suficiente regular la temperatura del reactor en $x_{2}^{*}$ (ver el capitulo 3 ).

Asúmase lo siguiente: (i) La salida $y=x_{2}$ tiene un ruido de medición correspondiente al $10 \%$ (éste ha sido exagerado para observar su efecto en el desempeño). (ii) Existe incertidumbre sobre la tasa y entalpía de reacción. (iii) Los parámetros $\theta, \gamma x_{1, e}$ y $x_{2, \varepsilon}$ son conocidos y constantes. Bajo las suposiciones anteriores, este problema de estabilización ya ha sido resuelto usando el CAR (43),(44) (ver sección 3.2). No obstante, se retoma con fines 
Capitulo Quinto

de comparación del desempeño obtenido con los controladores $(43),(80)$ y (43),(44).

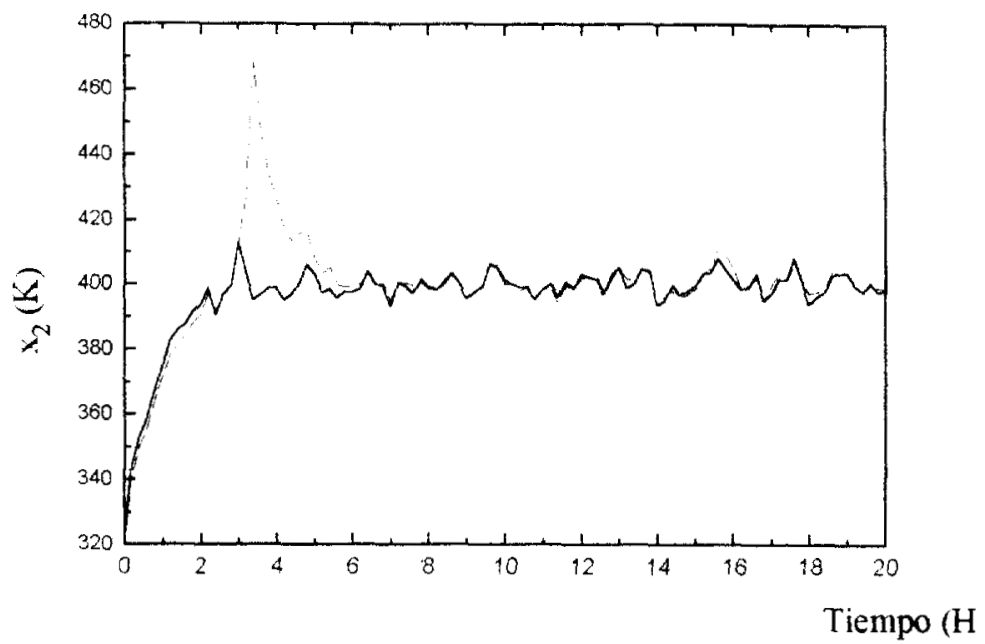

Figura 23. Estabiliazción de un RCTA exotérmico. Controlador (43),(44) (discontinua) y (43), (80) (continua).

Las simulaciones numéricas fueron hechas tomando los siguientes valores de los parámetros involucrados: $\theta=\gamma=1.0, x_{l, e}=1.0, x_{2, \mathrm{e}}=350, \mathrm{k}_{1}=\exp (25), \mathrm{Ea} / \mathrm{R}=1 \times 10^{4}, \beta(x)$ $=200$ y $u=350$. La ganancia de control $K_{1}=1.0$. Las constantes de estimación $\kappa_{1}$ y $\kappa_{2}$ fueron elegidas tales que el polinomio $s^{2}+\kappa_{2} s+\kappa_{1}=0$ es Hurwitz. El parámetro de ajuste $\mathrm{L}=18$. Cabe señalar que $\mathrm{L}>\mathrm{L}^{*} \approx 14.7$ con lo cual se garantiza la estabilidad a lazo cerrado (ver sección 3.2).

Por último, el control (43),(44) fue modificado en forma tal que $u^{\mathrm{s}}=\operatorname{Sat}\{\cdot\}=420 \mathrm{si}$ $u>420, u^{s}=S a t\{\cdot\}=380$ si $u<380$, y $u^{s}=u$ para cualquier otro caso. La ley de control dependiente de superficie deslizante no se satura. La ganancia constante $g_{d}=1$. En la Figura 23 se muestra el desempeño de ambos controladores. El control (43),(80) disminuye el sobredisparo mientras que el $(43),(44)$ exhibe mayor sensibilidad.

\subsection{Observaciones sobre las extensiones discutidas}

En este capitulo se han expuesto sólo algunas de las extensiones del algoritmo de control basado en estimación de incertidumbres aglutinadas. Se ilustraron algunas ventajas mediante la discusión de resultados preliminares. Todos estos casos están siendo estudiados sistemáticamente para reportar resultados significativos. Las propiedades de robustez y el desempeño logrado serán reportados en breve.

En el primer ejemplo, se abordó el problema de seguimiento de una referencia. Este resulta en el caso cuando el grado relativo no está bien definido. A consecuencia de esto la ley de control es no causal. Es decir, se requiere conocer la derivada del control para calcular 
la retroalimentación. Este inconveniente es similar al que presentan las plantas con retardo. Por supuesto que este hecho impone restricciones sobre el controlador. Sin embargo, tal como lo muestran las simulaciones, el algritmo resulta en una ley de control que consigue seguir algunas referencias. Esto se logra porque la derivada del control (desconocida) se aglutina en la función no lineal para después interpretar a ésta como una variable de estado en un sistema extendido. Luego, usando un estimador de estados se puede disponer de un estimado del estado aumentado. Así que se prove a la ley de retroalimentación de un estimado de las incertidumbres.

En el segundo ejemplo se muestra la regulación de temperatura de efluente de proceso en presencia de dinámica de actuador desconocida (dinámica no modelada). El caso simulado corresponde a un intercambiador de calor con alimentación a contracorrienete. Se llevaron a cabo simulaciones y el controlador ofrece buen desempeño y un margen de robustez aceptable. Lo anterior permite suponer que el uso del algoritmo propuesto resulta en controladores robustos para procesos con dinámicas no modeladas.

Además, se esbozó un método para modificar la ley de control basada en saturación del control con el fín de obtener una con dependencia en superficie deslizante. Con ésta, el desempeño de las acciones de control se mejoran cuando la medición está contaminada con ruido de medición. Se ejemplifica esta mejora con RCTA exotérmico (el cual es recurrentemente usado a lo largo del texto)

No sobra el mencionar que a la fecha se ha utilizado el algoritmo de linealización asintótica robusta en diferentes clases de sistemas. La supresión de caos en osciladores ha sido lograda tanto con el algoritmo continuo [42] como con el discreto [43] (aún cuando el orden de los osciladores sea mayor que uno). La sincronización robusta de osciladores [44] (a pesar de que los modelos de los osciladores sean estrictamente distintos) y el control de posición en sistema de levitación magneto-superconductor [45] (en este caso, se desconoce la fuerza magnética que supende al imán, sólo se sabe que exhibe histeresis y se conoce una expresión heurística de la dinámica de la fuerza magneto-superconductor). Sin embargo, éstos tampoco son incluidos en este texto por la misma razón, van más allá de los objetivos planteados en el presente proyecto. 


\section{Conclusiones Finales}




\section{Conclusiones finales}

En este trabajo de investigación se desarrolló un algoritmo de control para procesos químicos que es robusto ante errores en modelo, variaciones paramétricas y perturbaciones en la planta. Para ello, se aglutinan las incertidumbres en una función no lineal, la cual puede ser interptretada como una variable de estado. Luego, se constuye un sistema en espacio extendido. Es para el sistema en espacio extendido que se diseña un observador de estados. El estimador de estados provee a la ley de control linealizante con un estimado del nuevo estado (y por consecuencia de las incertidumbres). El esquema propuesto presenta un desempeño aceptable. El controlador consta de dos partes: (a) un compensador dinámico de incertidumbres, dado por un observador de alta ganacia y (b) una ley de control de tipo linealizante.

El estimador de incerticlumbres sólo tiene un parámetro de ajuste llamado ganacia de alta frecuencia, L. Se puede garantizar la estabilidad del sistema a lazo cerrado para un valor de $\mathrm{L}$ grande. Sin embargo, en la precencia de ruido de medición, esto puede acarrear dificultades de estabilidad. Adicionalmente, puesto que los estimados de incertidumbres son obtenidos a partir de un estimador de alta ganancia, se pueden presentar sobredisparos (principalmente en cambios en escalón de la referencia). Con el fin de disminuir el efecto de los sobredisparos en la salida de proceso, la retroalimentación puede ser modificada mediante una función de saturación. Empero, tal vez otras alternativas puedan ser viables. Quizá la estimación de estados mediante un filtro óptimo de Kalman, o bien, un observador diseñado en base a estructura variable para obtener un estimador de modo desliazante. Otra opción fue ilustrada en el último capitulo y consiste en modificar la ley de control y no el estimador de estados. La modificación explorada, hasta el momento, consiste en diseñar una retroalimentación dependiente de una superfice deslizante.

Por otra parte, el algoritmo ha sido presentado en la forma como fue desarrollado. Se asumieron restricciones que fueron relajadas en cada etapa del proyecto. Así, se muestra una forma constructiva de abordar el problema de control en sistemas reactantes desde el punto de vista de aglutinación de incertidumbres.

En primer instancia se consideró el problema de control de procesos (donde sólo existe errores en modelo) utilizando un esquema de estimación de incertidumbres basado en diferencias finitas centrales. El resultado es un controlador que depende únicamente de valores conocidos (la acción de control inmediata anterior y valores estimados de incertidumbres). Aunque sólo se garantizó estabilidad ante errores en modelo, una implementación experimental a escala piloto muestra que el CAR tiene propiedades de robustez ante perturbaciones en la alimentación del proceso. Además, la implementación experimental permite vislumbrar que la aplicación a mayor escala es viable. Tanto, que se requieren menos mediciones que con otras ya reportadas para ofrecer un desempeño similar.

Luego, se desarrolló un algoritmo de diseño basado en estimación continua. Como estimador se propuso un observador de tipo Luenberger. Esto fue muy provechoso para analizar la estructura del controlador. Una comparación en terminología clásica, permite 
entender al esquema diseñado como un controlador PI con estimación dinámica de incertidumbres. La estimación dinámica es provista por un término de acción integral al cuadrado, el cual resulta de la estimación obtenida por el estimador de incetidumbres aglutinadas. Aqui se relajan algunas suposiciones dando como resultado un controlador con robustez ante errores en modelo, variaciones paramétricas y perturbaciones en la planta. El desempeño del controlador resultante es aceptable y su implementación es ilustrada por medio de reactores exotérmicos.

El control asintótico robusto tiene estructura de tipo adaptativo puesto que comprende un esquema de estimación de incertidumbres y una aproximación a la retroalimentación de estados. Se recurre a un reactor biológico con modelo simple para llevar a cabo una comparación de desempeño entre dos algoritmos adaptativos, un PI-IMC y el CAR. El resultado es favorable para el esquema desarrollado en este proyecto. Este ofrece buena regulación y bajo esfuerzo de control.

Después de eso, se establece un algoritmo para el diseño de un controlador asintótico robusto para sistemas de $n$-ésimo orden. El análisis de estabilidad no es sencillo y está fundamentado fuertemente en resultados de linealización estrada-salida reportados en el último lustro. Sin embargo, quedan claras las propiedades robustas del CAR. Unos casos de estudio permiten explorar e ilustrar las características del control resultante: La estabilización de reactores exotérmicos, la regulación de sustrato en una clase de fermentador y la regulación de temperatura en efluente de procesos.

Todos los casos anteriores son usados para mostrar la robustez del CAR. El desempeño obtenido en cada uno de ellos satisface los requerimiento planteados al inicio del texto: Controlar el proceso quimico para mantener dentro de especificaciones (estabilidad práctica) a un estado de interés con acciones de control de bajo esfuerzo. Por medio de algunas extensiones a los resultados obtenidos se vislumbran las lineas de investigación a seguir y se relajan (al menos parcialmente) las suposiciones más drásticas.

El controlador asintótico robusto tiene un orden bajo. Sólo se requiere integrar $\rho+1$ ecuaciones diferenciales. Esto es una ventaja del CAR ante otro tipo de estrategias con estructura adaptativa.

Pero no todo es vida y dulzura, aún falta analizar la Fragilidad del controlador asintótico robusto diseñado a partir del algoritmo propuesto. Aunque este concepto ha sido dsicutido implicitamente tanto en congresos como conferencias especializadas en teoría de control, no es sino hasta recientemente que fue acuñado de forma clara [46]. Con la noción de fragilidad se expone la diferencia entre robustez, optimalidad y desempeño. Más aún, se puede reconocer que la robustez puede ocasionar que sistemas de control a lazo cerrado sean inestastables debido a posibles variaciones en el los parámetros de control, aún del order de $10^{-9}$.

La noción de fragilidad se fundamenta en la necesidad que algún controlador, que es parte de un sistema a lazo cerrdado, sea capaz de tolerar incertidumbre en sus coeficientes. 
Existen dos razones para esto. Primero, la implementación del controlador esta sujeta a la impresición inherente en la conversión analógica-digital-analógica asociada con: la longitud finita de la "palabra", la resolución finita de los instrumentos de medición y el error de redondeo en cálculos numéricos. Así, se requiere que exista un margen de tolerancia alrededor del control diseñado distinto de cero. Segundo, todo algoritmo de diseño de control requiere reajustes porque un índice no escalar puede capturar todos los requerimientos de desempeño de un sistema de control. Esto se traduce al requerimiento de que la estabilidad práctica y el margen de desempeño estén disponibles y asociados con la función de transferencia del controlador nominal (o bien, otros parámetros que caracterizen la implementación) [46].

Así pues, el estudio de la fragilidad de una ley de control es un nuevo campo de estudio, el cual complementa e incrementa las líneas de investigación dentro de la prespectiva de incertidumbres aglutinadas. 
Apendice A: Un resultado de estabilidad

A péndice A (Un resultado de estabilidad para una clase de sistemas variantes en tiempo)

Lema A.1: Considérese el siguiente sistema lineal variante en tiempo $e=\mathrm{A}(\kappa, r) e$, donde $\kappa=\left(\kappa_{1}, \kappa_{2}\right)$ es tal que $P_{2}(s)=s^{2}+\kappa_{1} s+\kappa_{2}=0$ es Hurwitz lo cual implica que $\kappa_{1}, \kappa_{2}$ $>0$. La matriz $A(\kappa, r)$ está dada por

$$
A(\kappa, r)=\left[\begin{array}{cc}
-\kappa_{1} & 1 \\
-r \kappa_{2} & 0
\end{array}\right]
$$

y $r=n(t)$ satisface $0<\varepsilon_{1} \leq r \leq \varepsilon_{2}<1$, entonces, el sistema lineal anterior es exponencialmente estable.

Prueba: Reescribamos al sistema lineal variante en tiempo de la siguiente forma:

$$
\dot{e}=\hat{A}(\kappa) e+B(r) e
$$

donde

$$
\hat{A}=\left[\begin{array}{ll}
-\kappa_{1} & 1 \\
-1 & 0
\end{array}\right], \quad B(r)=\left[\begin{array}{cc}
0 & 0 \\
1-\kappa_{2} r & 0
\end{array}\right]
$$

dado que $\mathrm{P}_{\text {. }}(\mathrm{s})$ es Hurwitz, $\kappa_{1}>0$, por consiguiente la matriz A es Hurwitz. Entonces existe una matriz invertible $\mathrm{P}$ tal que el sistema (A.2) puede ser escrito como $e=\mathrm{P}^{-1} \mathrm{JP} e+\mathrm{B}(r) e$, donde $\mathrm{J}$ es la forma canónica de Jordan de $\mathrm{A}(\kappa)$. Ahora, considérese el cambio de coordenadas $\varepsilon^{\prime}=e \mathrm{P}$, así se puede tansformar $\dot{e}=\mathrm{P}^{-1} \mathrm{JP} e+\mathrm{B}(r) e$ a

$$
\dot{\varepsilon}^{\prime}=J \varepsilon^{\prime}+\bar{B}(r) \varepsilon^{\prime}
$$

donde $\overline{\mathrm{B}}(r)=\mathrm{PB}(r) \mathrm{P}^{-1}$. Luego, existe una norma \|\| tal que $\left\|\varepsilon_{0}^{\prime} \exp (\mathrm{Jt})\right\| \leq \exp (-\mathrm{t})\left\|\varepsilon_{0}^{\prime}\right\|$. De este modo la integral del sistema (A.4), está dada por

$$
\left\|\varepsilon^{\prime}(t)\right\| \leq \exp (-t)\left\|\varepsilon_{0}^{\prime}\right\|+\exp (-t) \int_{0}^{t} \exp (\sigma)|\bar{B}(r(\sigma))|\left\|\varepsilon^{\prime}\right\| d \sigma
$$

Adicionalmente, $\quad \overline{\mathrm{B}}(r)|\leq| \mathrm{P}^{-1}|| \mathrm{P}|| \mathrm{B}(r) \mid \leq 1-\mathrm{K}_{2} r \leq 1-\varepsilon_{1}$. Por lo tanto,

$$
\left\|\varepsilon^{\prime}(t)\right\| \leq \exp (-t)\left\|\varepsilon_{0}^{\prime}\right\|+\left(1-\varepsilon_{1}\right) \exp (-t) \int_{0}^{t} \exp (\sigma)\left\|\varepsilon^{\prime}\right\| d \sigma
$$

A partir del lema de Bellman-Gronwall [19], se tiene que $\left\|\varepsilon^{\prime}(t)\right\| \leq\left\|\varepsilon^{\prime}\right\| \exp \left(-\varepsilon_{1} t\right) \leq 0$. 
Apéndice B: Un resultado acerca e la obsreservabilidad robusta

Apéndice B (Un resultado de acerca de la observabilidad robusta) [28].

Considérese el sistema $\dot{z}=f(z, \varepsilon, w)$ con $\varepsilon=0$. Asúmase ${ }^{5}$ que existe un conjunto abierto $\mho_{1}$ en $\mathbf{R}^{\mathrm{p}}$, un número real nonegativo $\mathrm{v}<1$, un número real $a \geq 1$ y una función $C^{\mathrm{i}} \mathrm{V}$ : $\mho_{1} \rightarrow \mathbb{R}_{+}$tal que el conjunto $\mathrm{Z}=\{\mathrm{z}: \mathrm{V}(\mathrm{z}) \leq a+1\}$ es un subconjunto compacto de $\mho_{1}, \mathrm{y}$ tenemos $V \leq-\Upsilon_{1}(z)$ donde $\Upsilon_{1}(z)$ es continua sobre $\mho_{1}$ y positiva definida sobre $Z$.

Observación B.1: En la ausencia de $w=w(\mathrm{t})$, si el equilibrio $\mathrm{z}=0$ de $\dot{z}=f(\mathrm{z}, 0)$ es localmente asintóticamente estable con dominio de atracción $\mho_{1}$, se puee proveer una función de Lyapunov suave que satisfaga la suposición PLU. Más aún, $\cup$ puee ser elegido igual acero y $a$ puede ser elegido arbitariamente grande (para más detalles ver [19]).

Lema B.2: Considérese el siguiente sistema no lineal

$$
\begin{aligned}
& \dot{z}=F(z, \varepsilon, w) \\
& \dot{\varepsilon}=L A \varepsilon+\Phi(z, \varepsilon, w)
\end{aligned}
$$

donde $z \in \mathbf{R}^{p}, \varepsilon \in \mathbf{R}^{p+1}$ y $L$ es un número real estrictamente positivo. Asúmase que la propiedad PLU se satisface y sea $Z=\{z: V(z) \leq a+1\}$. Supóngase además que la matriz $A$ es Hurwitz y que existen unos números positivos reales $\sigma_{1}$ y $\sigma_{2}$ y una cota continua $\delta_{1}$ con $\delta_{1}(0)=0$ que satisfacen

$$
\left.\begin{array}{rl}
|f(z, \varepsilon, w)|-f(z, 0, w) \mid & \leq \delta_{1}(|\varepsilon|) \\
|\Phi(z, \varepsilon, w)| & \leq \sigma_{1}+\sigma_{2}|\varepsilon|
\end{array}\right\} \forall(z, \varepsilon, w) \in Z \times \mathbb{R}^{\rho+1} \times W
$$

Sea $q(L)$ una función de clase $K_{\infty}$ que satisface

$$
\operatorname{Liminf}_{L \rightarrow \infty} \frac{L}{q^{4}(L)} \rightarrow \infty
$$

Adicionalmenta, sea $P$ la solución de la ecuación matricial $A^{\mathrm{T}} P+P A=-1$ y finalmente definase a la función

$$
D(z, \varepsilon)=a \frac{V(z)}{a+1-V(z)}+q(L) \frac{\ln \left(1+\varepsilon^{T} P \varepsilon\right)}{q(L)+1-\ln \left(1+\varepsilon^{T} P \varepsilon\right)}
$$

y el conjunto

${ }^{5}$ El número " $1 "$, de aqui en adelante se'ra arbitrario y podría ser remplezado por cualquier número estrictamente positivo. Esta suposición es conocida como: Propiedad Lyapunov Uniforme (PLU) 
Apéndice B: Un resultado acerca e la obsreservabilidad robusta

$$
\mho_{2}=\{z: V(z)<a+1\} \times\left\{\varepsilon: \ln \left(1+\varepsilon^{T} P \varepsilon\right)<q(L)+1\right\}
$$

Bajo las condiciones (B.1)-(B.5), para cadaa número real estrictamente positivo L, la función $D(z, \varepsilon): \mho_{2} \rightarrow \mathbb{R}$ es propia sobre $\mho_{2}$. También, para cada número real estrictamente positivo $a_{1}$, existe un número real $\mathrm{L}^{*}$ tal que para todo $\mathrm{L} \geq \mathrm{L}^{*}, D(\mathrm{z}, \varepsilon)$ satisface

$$
\dot{D} \leq-\Phi_{1}(z, \epsilon)
$$

donde $\Phi_{1}(\mathrm{z}, \varepsilon)$ es continua sobre $\mho_{2}$ y positiva definida sobre el conjunto $Z_{\mathrm{D}}=\left\{(\mathrm{z}, \varepsilon)\right.$ : $\mathrm{v}+a_{1}$ $\left.\leq D(\mathrm{z}, \varepsilon) \leq a^{2}+\mathrm{q}(\mathrm{L})+\mathrm{l}\right\}$.

Prueba: Empecemos la prueba remplazando $\ln \left(1+\varepsilon^{\mathrm{T}} P \varepsilon\right)$ in (B.4) por la función tal que $\Gamma(\varepsilon) \leq(\mathrm{q}(\mathrm{L})+1)\left[a^{2}+\mathrm{q}(\mathrm{L})^{2}+1\right] /\left[a^{2}+1+\mathrm{q}(\mathrm{L})^{2}+\mathrm{q}(\mathrm{L})\right]$. Asúmase que $D(\mathrm{z}, \varepsilon) \leq a^{2}+$ $\mathrm{q}(\mathrm{L})+1$. De las definiciónes de la función $\Gamma(\varepsilon)$ y el conjunto $Z=\{\mathrm{z}$ : $\mathrm{V}(\mathrm{z}) \leq a+1\}$ se implica que, para algún $L, z$ está en $Z$. Por consiguiente, a paritr de la suposición PLU y las cotas en (B.2) podemos escribir lo siguiente

$$
\left.\begin{array}{c}
\dot{V} \leq-\Upsilon_{1}(z)+\beta_{2} \delta_{1}|(\varepsilon)| \\
\dot{\Gamma}(\varepsilon) \leq \frac{1}{1+\varepsilon^{T} P \varepsilon}\left[-L|\varepsilon|^{2}+2 \lambda_{\max }(P)|\varepsilon|\left(\beta_{2}|\varepsilon|+\beta_{1}\right)\right]
\end{array}\right\} \forall(z, \varepsilon, w) \in Z \times \mathbf{R} \times W
$$

donde $\beta_{\mathrm{i}}$ 's son números positivos reales los cuales acotan $\partial \mathrm{V} / \partial \mathbf{z}$ sobre el conjunto $\mathrm{Z}$. Tales cotas existen porque $\mathrm{V}$ is $C^{1}$ y $\mathrm{Z}$ es compacto. Entonces, de (B.4), y (B.7) se tiene que

$$
\dot{D} \leq \frac{a(a+1)}{(a+1-\eta)^{2}}\left[-\Upsilon_{1}(z)+\beta_{3} \delta_{1}(|\varepsilon|)\right]+\frac{q(q+1)}{(q+1-\Gamma)^{2}} \frac{1}{1+e^{t} P_{\varepsilon}}\left[-L|\varepsilon|^{2}+2 \lambda_{\max }(P)|\varepsilon|\left(\beta_{2}|\varepsilon|+\beta_{1}\right)\right]
$$

Ahora fijemos $\mathrm{L}^{*}$, tal que $\mathrm{q}\left(\mathrm{L}^{*}\right)=a^{2}+a+1$. Este $\mathrm{L}^{*}$ existe porque $\mathrm{q}(\mathrm{L})$ es de clase $\mathrm{K}_{\infty}$. Luego, usando $a \geq 1$, la suposición PLU y eligiendo $\mathrm{L} \geq \mathrm{L}^{*}$ tenemos que $1 / 2 \leq a(a+1)$ / $(a+1 V)^{2} \leq 2 q^{4}$, con ello se puede reescribir (B.8) como

$$
\dot{D} \leq \frac{a(a+1)}{(a+1-V)^{2}}\left[-\Upsilon_{1}(z)+\beta_{3} \delta_{1}(|\varepsilon|)\right]+\frac{q(q+1)}{(q+1-\Gamma)^{2}} \frac{1}{1+e^{t} P e}\left[\frac{-L}{2 q^{4}}|\varepsilon|^{2}+4 \lambda_{\max }(P)|\varepsilon|\left(\beta_{2}|\varepsilon|+\beta_{1}\right)\right]
$$

Dado que la relación $[a(a+1)] /\left[(a+1-V)^{2}\right]$ es positiva y está acotada sobre cero en $Z$, es suficiente considerar la siguiente expresión 
Apéndice B: Un resultado acerca e la obsreservabilidad robusta

$$
-\Upsilon_{1}(z)+\beta_{3} \delta_{1}(|\varepsilon|)+\frac{q(q+1)}{(q+1-\Gamma)^{2}} \frac{1}{1+\varepsilon^{t} P e}\left[\frac{-L}{2 q^{4}}|\varepsilon|^{2}+4 \lambda_{\max }(P)|\varepsilon|\left(\beta_{2}|\varepsilon|+\beta_{1}\right)\right]
$$

Estamos interesados en evaluar a la expresión anterior sobre el conjunto

$$
\Lambda_{L}=\left\{(z, \varepsilon): v+a_{1} \leq D(z, \varepsilon) \leq a^{2}+q^{2}(L)+1\right\}
$$

y lo haremos considerando los dos conjuntos: $\Lambda_{1}=\{(\mathrm{z}, \varepsilon): \mathrm{V}(\mathrm{z}) \leq a+1,1<\Gamma(\varepsilon)<\mathrm{q}(\mathrm{L})+\mathrm{l}\}$ y $\Lambda_{0}=\left\{(\mathrm{z}, \varepsilon: \mathrm{V}(\mathrm{z}) \leq a+1, \Gamma(\varepsilon) \leq 1\} \cap\left\{(\mathrm{z}, \varepsilon): \mathrm{u}+a_{1} \leq[a \mathrm{~V}(\mathrm{z})] /[a+1-\mathrm{V}(\mathrm{z})]+\Gamma(\varepsilon)\right\} \mathrm{y}\right.$ observando que $\Lambda_{\mathrm{L}}$ está contenido en $\Lambda_{1} \cup \Lambda_{0}$. Dado que tenemos $\left\{\Gamma(\varepsilon) \leq 1, \mathrm{v}+a_{1} \leq D(z, \varepsilon)\right\}$ $\Rightarrow v+a_{1} \leq[a V(z)] /[a+1-V(z)]+\Gamma(\varepsilon)$.

En el conjunto $\Lambda_{1}$, nótese que el límite daado en (B.3) se mantiene, z está contenida en un conjunto compacto independiente de $L,[q(q+1)] /[q+1-\Gamma(\varepsilon)]$ está acotada sobre cero y la función $\delta_{1}(|\varepsilon|)$ está acotada. No se usa la cota superior de $[q(q+1)] /[q+1-\Gamma(\varepsilon)]$, la cual depende de L. Finalmente, por examinación de la expresión (B.9a), se sigue que existe un número real positivo $\mathrm{L}_{2}^{*}$ tall que para cada $\mathrm{L} \geq \mathrm{L}_{2}^{*}$ la función dada en (B.9) puede estar acotada por arriba por una función de $(z, \varepsilon)$ la cual es negativa definida sobre $\Lambda_{1}$.

En el conjunto $\Lambda_{0}$, Para verificar que $\mathrm{d} D / \mathrm{dt}$ es negativa definida para todo $(\mathrm{z}, \varepsilon, w) \in$ $\Lambda_{0} \times W$, notemos que para $\mathrm{L} \geq \mathrm{L}$ ' tenemos

$$
\min _{0 \leq \Gamma \leq 1}\left\{\frac{q(q+1)}{(q+1-\Gamma)^{2}}\right\}=\frac{q}{q+1}, \quad \max _{0 \leq \Gamma \leq 1}\left\{\frac{q(q+1)}{(q+1-\Gamma)^{2}}\right\} \leq 2
$$

Se sigue que para conocer el signo de la expresión (B.9a), lo aseguramos tomando

$$
\begin{aligned}
x & =\varepsilon \\
K & =L \\
\chi(z) & =\frac{1}{2} \Upsilon_{1}(z) \\
\mathrm{Q}(\varepsilon) & =\frac{1}{2} \frac{\varepsilon^{T} \varepsilon}{\left(1+\varepsilon^{T} P \varepsilon\right)} \\
\varphi(z, \varepsilon) & =\beta_{3} \delta_{1}(|\varepsilon|)+* \lambda_{\max }(P)|\varepsilon|\left(\beta_{2}|\varepsilon|+\beta_{1}\right)
\end{aligned}
$$

y una función $g(\cdot)$ de clase $K_{\infty}$ que satisface $g(L) \leq L /\left[2 q^{3}(q+1)\right]$. Tal función existe porque $L /\left[2 q^{3}(q+1)\right]>0$ para $L>0$ y el límite (B.3) se mantiene. El conjunto $\Lambda_{0}$ es compacto e independiente de $\mathrm{L}$. Sus proyecciones $\Lambda_{0} \subset \Lambda_{0, z} \times \Lambda_{0, \varepsilon}$ satisfacen $\Lambda_{0, z} \subset\{\mathrm{z}: \mathrm{V}(\mathrm{z}) \leq a+1\}=Z$ y $\Lambda_{0, \varepsilon} \subset\{\varepsilon: \Gamma(\varepsilon) \leq 1\}$. entonces, a partir de (B.11) y las propiedades de $\Upsilon_{1}(z), \chi(z)$ es positiva 
Apéndice B: Un resultado acerca e la obsreservabilidad robusta

definida sobre $\Lambda_{0, z}$ y $\mathbf{Q}(\varepsilon)$ es continua sobre $\Lambda_{0, \varepsilon}$. Evidentemente, $\varrho(\varepsilon)$ es positiva definida sobre $\Lambda_{0, \varepsilon}\{0\}$. También, de la continuidad de $\delta_{1}$ y el hecho que $\delta(0)=0, \varphi(z, \varepsilon)$ es continua sobre $\Lambda_{0}$ y $\varphi(z, \varepsilon)=0$ para todo $(z, \varepsilon) \in\{(z, \varepsilon): \varepsilon=0\} \cap \Lambda_{0}$.

Para ver que $\chi(z)$ es positiva definida sobre la proyacción del conjunto $\{(z, \varepsilon): \varepsilon=0\}$ $\cap \Lambda_{i}$, tenemos que, con $0 \leq \mathrm{v} \leq 1$ y $a_{1}>0$,

$$
\left\{\varepsilon=0, \mathrm{u}+a_{1} \leq \frac{a V(z)}{a+1-V(z)}+\Gamma(\varepsilon)\right\}-\mathrm{u}<V(z)
$$

Así que de la suposición PLU, $\chi(z)$ es positiva definida sobre la proyección de $\{(z, \varepsilon): \varepsilon=$ $0\} \cap \Lambda_{0}$. Por consiguiente, existe un número reeal positivo $L_{2}^{*}$ tal que, para $L \geq L_{2}^{*}$, la expresión (B.9a) puede estar acotada por arriba sobre $\Lambda_{0}$ por la función - $1 / 2 \Upsilon_{1}(z)-g(L) \varrho(\varepsilon)$ la cual es negativa definida sobre $\Lambda_{0}$ ya que $\mathrm{g}(\varepsilon)$ es positiva definida. Por último, tomemos entonces $\mathrm{L} \geq \max \left\{\mathrm{L}^{*}, \mathrm{~L}_{1}{ }_{1}, \mathrm{~L}_{2}{ }_{2}\right\}$. 
Bibliografia

\section{Bibliografía}

[1] A. Isidori (1989), Nonlínear Control Systems, Springer-Verlag, Berlin; H. Nijmeijer y A. van der Schaft (1990), Nonlínear Dynamical Control Systems, Springer-Verlag, NY.

[2] R. Aris (1969), Elementary Chemical Reactor A nalysis, Prentice-Hall, New Jersey.; G. R. Gavalas (1968), Nonlinear Differential equations of Chemical Reacting Systems. Springer-Verlag, N.Y.

[3] J. Alvarez-Ramírez, R. Suárez y R. Femat, Control of Continuous-Stirred Tank Reactors: Stabilization with Unknown Reaction Rates, Chem. Eng. Sci., 51, 4183-4188.

[4] J. Alvarez-Ramírez, R. Femat, O. Monroy, y F. Cuervo, An Adaptive Strategy for Anaerobic Digester, $5^{\text {th }}$ IEEE Int. Conf. on Control Appl. Septiembre de 1996, Dearborn, Michigan.;O. Monroy, J. Alvarez-Ramirez y R. Femat, Mass Balance

Strategy for the Robust Control of Anaerobic Digestion, $I^{\text {st }}$ Workshop on Monitoring and Control of Anaerobic Digestion Processes, Diciembre, 1995, Narbone, Francia

[5] J. Alvarez-Ramírez, R. Femat y A. Barreiro, A PI ontroller with Dynamic Disturbance Estimation, Ind. Eng. Chem. Res., por aparecer.

[6] J. Alvarez-Ramírez, R. Suárez y R. Femat, Robust Stabilization of Temperature in Continuous Stirred Tank Reactors, Chem. Eng. Sci., 52, 2223.

[7] J. Alvarez-Ramírez, R. Femat y J. González-Trejo (!997), Robust Control of Uncertain First-Order Systems, Chem. Eng. Sci., por aparecer.

[8] J. Alvarez-Ramírez, I. Cervantes y R. Femat (1997), Robust Controllers for a Heat Exchanger, Ind. Eng. Chem. Res., 36, 382.

[9] A. Uppal, W.H. Ray y A.B. Poore (1974), On the Dynamical Behavior of Continuous

Stirred Tank Reactors, Chem. Eng. Sci., 29, 967.

[10] L.P. Russo y B.W. Bequette (1995), Impact of Process Design on the Multiplicity Behavior of a Jacketed Exothermic CSTR, AIChE J., 41, 135.

[11] O. Paladino, M Ratto y P. Costa (1995), Chaos and Chemical Models: Sensivity of dynamics parameters uncertainty, Chem. Eng. Sci., 50, 3829.

[12] D.G. Retzloff, P. C-H. Chan, C. Chicone, D. Offin y R. Mohamed (1987), Chaotic Behavior in the Dynamical System of a Continuous Stirred Tank Reactor, Physica D, 25,131 .

[13] J. Alvarez-Ramírez (1994), Stability of a Class of Uncertain Continuous Stirred Tank Reactors with a Nonlinear Feedback, Chem. Eng. Sci., 49, 1743. 
Bibliografia

[14] B. R. Barmish, M. Corless y G. Leitman (1983), A New Class of Stabilizing Controllers for Uncertain Dynamical Systems, SIAM J. Control Optim., 21, 246.

[15] P. Doutidis, M. Soroush y C. Kravaris (1990), Feedforward/feedback Control of Multivariable Nonlinear Process, AIChE J., 36, 1471.

[16] D.T. Hill y C.L. Barth (1977), A Dynamic Model for Simulations of Animal Waste Digestion, J. Water Pollut. Control Fed, 7, 2119; D. Dochain y G. Bastin (1986), Modelling and Control of Biotechnological Processes, Pergamon Press, Oxford, UK; P. Renard, D. Dochain y G. Bastin (1988), Adaptive Control of Anaerobic Digestion Processes: A pilot scale application, Biotech. and Bioeng., 31, 287.

[17] O. Monroy, J. Alvarez-Ramírez, F. Cuervo y R. Femat (1996), An Adaptive Strategy to Control Anaerobic Digesters for Watewater Treatment, Ind. Eng. Chem. Res., 35, 3442 .

[18] V. Arnold (1978), Mathematical Methods of Classical Mechanics, Springer-Verlag, NY.

[19] A. Teel y L. Praly (1995), Tools for Semiglobal Stabilization by Partial State and Output Feedback, SIAM J. Control Optim., 33, 1143.

[20] D.R. Coughanowr y L.B Koppel (1965), Process Systems Analysis and Control, McGraw-Hill, USA.W. L. Luyben (1989), Process Modeling Simulation and Control for Chemical Engineerings $2^{\text {nd }}$ Ed., McGraw-Hill, Singapore. G. Stephanopoulos, C.A. Smith y A. B. Corripio (1991), Control A utomático de Procesos, Noriega-Limusa, México. G. Stephanopoulos (1984), Chemical Process Control, An Introduction to Theory and Practice, Prentice-HAll, USA.

[21] S. Skogestad y M. Morari (1988), LV-control of High-purity Distillation Columns, Chem. Engng. Sci., 43, 33.

[22] I. L. Chien y P.S. Fruehauf (1990), Consider IMC Tuning to Improve Control Performance, AIChE J., 86, 33 .

[23] M. Morari y E. Zafiriou (1989), Robust Process Control, Prentice-Hall, NJ USA.

[24] S. Rönnbäck (1993), Linear Control of Systems with Actuator Constraints, Tesis Doctoral, Universidad Tecnológica de Luleå, Suecia.

[25] R. Femat, J. Alvarez-Ramirez, M. Rosales-Torres y R. Garrido-Moctezuma (1997), Robust Asymptotic Regulation of Susbtrate in Biological Reactors: Design and performance comparison, Ind. Eng. Chem Res., En revisión.

[26] J.D. Bošković (1995), Stable Adaptive Control of a Class of First Order NonlinearlyParametrized Plants, IEEE Trans. on Aut. Control, 40, 347; J.D. Bošković (1996), Stable Adaptive Control of Class of Continuous Flow Bioreactor, $A I C h E J ., \mathbf{4 2}, 176$. 
Bibliografia

[27] J.D. Bošković, K.S. Narendra (1995), Comparison of Nonlinear, Linear and NeuralNetwork-Based Adaptive Controllers for a Class of Fed-Batch Fermentation, A utomatica, 31, 817.

[28] F. Esfandiari y H.K. Khalil (1992), Output Feedback Stabilization of Fully Linearizable Systems, Int. J. of Control, 56, 5, 1007; H.K. Khalil (1996), Adaptive Output Feedback Control of Nonlinear Systems Represented by Input-Output Models, IEEE Trans. on A ut. Control, 41, 2, 177.

[29] L. Hsu, A. D. de Araújo y R.R. Costa (1994), Analysis and Design of I/O Based Variable Structure Adaptive Control, IEEE Trans. on A ut. Control, 39, 1.

[30] A. Tornambè (1992), Output Feedback Stabilization of a Class of Non-minimum Phase Systems, Syst. and Control Letts, 19, 193-204.

[31] A. Isidori y C.I. Bymes, Output Regulation of Nonlinear Systems, IEEE Trans. on A ut. Control, 35, 2, 135 .

[32] J. Alvarez-Ramírez, R. Suárez y R. Femat (1996), Robust Control of a Class of Uncertain Nonlinear Systems in Strict-Feedback Form, European J. of Control, En revisión.

[33] R. Ray (1981), New approaches to the Dynamics Nonlinear Systems with Implications for Process Control Systems Design, Procc. Chem. Process Control, 245.

[34] H. J. Sussman and P.V. Kokotovic (1991), The Peaking Phenomenon and the Global Stabilization of Nonlinear Systems, IEEE Trans. on Aut. Control, 36, 424.

[35] J.H. Su (1994) Comments on "Satbility Margin Evaluation of Uncertain Linear Systems", IEEE Trans. A ut. Control, 39, 2523

[36] I. Kanellakopoulos, P.V. Kkotovic, P. Morse (1992), Adaptive Output Feedback Control of Systems with Output Nonlinearities, IEEE Trans. A ut. Control, 1166.

[37] H. Aoufousi, M. Perrier, J. C., Chavarie y D. Dochain (1992), Feedback Linearizing Control of a Fluidized Bed Reactor, Can. J. of Chem. Eng., 70, 356.

[38] T. Takamatzu, S. Shiya y Y. Okada (1985), Profile Control Scheme in a Baker's yeast Fed-Batch Culture, Biotech. and Bioeng., 27, 1675.

[39] Y.S.N. Malleswararao y M. Chidambaram (1992), Nonlinear Controllers for a Heat Exchanger, J. Procress Control, 2, 17.

[40] M.H.R. Fazlur Rahman y R. Devanathan (1995), Feedback Linearization of a Heat Exchanger, Syst. and Control Letts., 26, 203. 
Bibliografia

[41] H. Sira-Ramirez y O. Llanes-Santiago (1996), On the Stabilization of Nonlinear Systems via Input-Dependent Sliding Mode Surfaces, 6, 771.

[42] R. Femat, J. Alvarez-Ramírez y J. González (1997), A srategy to Control Chaos in Nonlinear Driven OScillators with Least Prior Knowledge, Phys. Letts. A, 224, 271.

[43] J. Alvarez-Ramírez, R. Femat y J. González (1996), A Time delay Strategy to control a class of chaotic oscillators, Phys. Letts. A 211, 41.

[44] R. Femat y J. Alvarez-Ramírez (1997), Synchronization of a Class of Strictly Different Chaotic Oscillators, Phys. Letts A. (por aparecer).

[45] R. Femat (1997), A Control Scheme for the Motion of a Magnet Supported by Type-II Superconductor, Physica D, (por aparecer).

[46] L.H. Keel y S. P. Bhattacharyya (1997), Robust, Fragile or Optimal ?, IEEE Trans. on A ut. Control, 42, 1098. 
Indice alfabetico

\section{Indice}

Alta ganancia

desempeño 17

estabilización meditante, 16

CAR

control asintótico robusto 6

Compensador dinámico

discreto 18

Control proporcional integral

sintonización en base a modelo interno 39

Control robusto

asintótico 29

definición 5

Digestión anaerobia 5

modelo dinámico 23

tratamiento de aguas residuales

$$
22
$$

Dinámica no modelada

control robusto en presencia de

84

definición 84

ejemplos típicos 84

Errores de modelado 4

Estabiliazdor práctico

global, para plantas de primer orden 34

Estabilidad interna

de señales en esquema continuo 32

de señales en esquema discreto 19

definición 12

Estabilidad práctica definición 12

Estabilidad semiglobal asintótica de un sistema a lazo cerrado 59

Estabilización asintótica

por retroalimentación de estados 52

Estabilización práctica

definición 12,16

semiglobal, definición 31

semiglobal, noción 31

Estabilizador práctico 6

de alta ganacia 17

de alta ganacia para el RCTA 16

ver definición de estabilización práctica 12,16

Estabilizador semiglobal asintótico

en un punto de equilibrio nominal 59

Estabilizador semiglobal práctico para sistemas de primer orden 33

Estimador de incertidumbres

Compensador dinámico 5 discreto, internamente estable 19

Fase minima 6

definición 12

en reactor de lecho fludizado, prueba 62

en un fermentador de lote alimentado, prueba 68

Forma canónica sistemas de orden superior 52

Función aglutinante estimación 31,55

Función aglutinante no lineal definición para plantas de primer orden 30

Función no lineal aglutinante en sistemas de orden superior 54

Ley de control

linealizante por retroalimentación de estados 53

Linealización entrada-salida

para sistemas de oreden superior 53

Lipschitz, función

definición 12

Modelo dinámico

de intercambiador de calor 72

de un digestor anaerobio 23

de un fermentador de lote alimentado 67

para la oxidación de Benceno 60

Multiplicidad de estados estacionarios

En un RCTA, Ejemplo 11

Objetivo de control

definición del 30

en digestion anaerobia 23

estabilización en un RCTA no isotérmico 21

estabilización práctica de un RCTA 30

Perturbaciones exógenas 4 vector de señales exógenas 29

Planta de prime orden sintonización 39

Planta de primer orden 
Indice alfabetico

como modelo de procesos 39

Primer integral

para planta de primer orden 30

Promedio efectivo de temperatura

en unidades de intercambio energético 72

Reactor continuo de tanque agitado

Esquema 10

modelo dinámico 10

polimerizacion de metil-metacrilato 20

Reactores de polimerización 5

Reatroalimentación de estados

para un RCTA no isotérmico 16

Regulación de temperatura

Estabilización de un RC'TA no isotérmico 21

Retroalimentación de estados

linealiazción por, sistemas de orden superior 51

Sistema dinamicamente extemamente equivalente

noción de invarianza 30

noción geométrica 55

Sistema extendido 6

$(\rho+1)$-dimensional 54

2-dimensional 31

para plantas de primer orden 30

Tasa de reacción

Propiedades 15

Valores nominales

a partir de diseño 6

Variaciones parametricas 4 


\section{Notación}

$A(\kappa, r)$ : Matriz compañera de error escalado de estimación.

a: Cocticiente constate

$\mathrm{C}(\mathrm{s}): \quad$ Operador de transterencia del control.

$\mathrm{C}_{\mathrm{T}}$ : Coeficiente total de transferencia de calor (en intercambiador).

d: Perturbaciones exógenas.

$f(x)$ : Campo vectorial incierto.

$\mathrm{G}(\mathrm{s})$ : Operador de transferencia entrada-salida de proceso.

$\mathrm{g}(x)$ : Campo vectorial incierto asociado a la entrada de control.

K: Ganancias de control.

$\mathrm{K}_{\mathrm{p}}$ : Ganacia estática de la planta.

L: Parámetro de alta ganancia de estimación.

$\mathrm{m}$ : Tamaño del vector de composiciónes en RCTA no isotérmicos.

n: Orden del modelo del proceso.

$\mathrm{P}_{\mathrm{n}}(\mathrm{s})$ : Polinomio Hurwitz de orden $\mathrm{n}$.

$R(x)$ : Tasa de conversión.

$r$ Relación entre la información asociada con la entrada de control desconocida sobre conocida.

$\mathrm{T}$ : Tiempo de retardo.

$\mathrm{t}$ : Tiempo.

U(s): Entrada de control (dominio de frecuencia).

u: $\quad$ Entrada de control.

$w$ : Vector de perturbaciónes.

$x$ : Vector de estados del modelo del proceso.

$Y(s)$ Salida del proceso (dominio de frecuencia).

$y$ : Salida el proceso (medición).

z: Vector de estados de sistema en forma canónica.

\section{Letras griegas}

$\alpha(z, v)$ : Función no lineat incierta.

$\beta$ : $\quad$ Número finito positivo.

$\beta(x)$ : Entalpía de reacción.

$\gamma(\mathrm{z}, \mathrm{v})$ : Función no lineal inciuerta asociada con entrada de control.

$\gamma(z)$ : Estimado de $\gamma(z, v)$.

$\Delta x$ : Promedio efectivo de diferencia de temperatura enún intercambiador de calor.

$\varepsilon$ : Error de estimación.

$\Delta \mathrm{T}: \quad$ Periodo de muestreo

$\delta(z, v)$ : Función de desviación de incertidumbre asociada a la entrada de control.

$\zeta(z, v)$ Dinámica interna. $\eta$ : Estado aumentado (incertidumbres aglutinadas como variable de estado).

$\Theta(z, v)$ : Función no lineal aglutinante de incertidumbres.

K: Constantes de estimación.

$\hat{\lambda}: \quad$ Polo de planta.

$\mu(x)$ : Tasa especifica de crecimiento de biomasa.

$v$ Vector de estados no observables.

: $\quad$ Preexponencial cinética.

$\pi \quad$ Conjunto de parámetros.

p: Grado relativo.

$\tau$ : Tiempo característico de la planta

$\tau_{\mathrm{c}}$ : Tiempo característico del control.

$\chi:$ Vector de temperaturas en un intercambiador de calor.

$\Psi$ : Condición de invarianza.

$\Omega$ : $\quad$ Región de atracción.

$\omega:$ Frecuencia de la señal de perturbación exógena.

Lista de abreviaturas

CAL: Control adaptativo lineal.

CANL: Control adaptativo no lineal.

CAR: Control asintótico robusto.

COD: Demanda quimica de oxigeno (en Ing;és).

IMC: Aproximación por modelo interno (siglas en inglés).

PI: Control proporcional integral.

RCTA: Reactor continuo de tanque agitado.

RIF: Reactor de lecho fluidizado.

SISO: Una entrada una salida (en inglés).

PLU: Suposición de propiedad Lyapnov uniforme 